\title{
Navigation and Control of Flexible Wing UAV Using Vision System
}

\author{
by \\ Malik Mohammed Ali Al-Isawi, M.Sc. \\ A thesis submitted to the Faculty of Graduate and Postdoctoral Affairs \\ in partial fulfillment of the requirements for the degree of
}

Doctor of Philosophy in Mechanical Engineering

Ottawa-Carleton Institute for Mechanical and Aerospace Engineering (OCIMAE)

Department of Mechanical and Aerospace Engineering

Carleton University

Ottawa, Ontario

June, 2018

(C) Copyright

Malik Mohammed Ali Al-Isawi, 2018 


\section{Abstract}

This thesis presents an advanced guidance and control system for unmanned aerial vehicles (UAV) with flexible wing. The control system is based on a stereo vision system and advanced fuzzy logic algorithms that can detect wing deflections and shapes. In general, this sensor has the capability, reliability, and performance of similar traditional control sensors, and provides reliable visual information that is useful for designing different control systems. The thesis proposes a novel Deflection-DetectionVision-System (DDVS) to control a flexible wing of unmanned aerial vehicle (UAV). The technique measure the deflection of the flexible wing with a stereo camera and determines the three-dimensional (3D) coordinates to identify the wing shape. In addition, the fuzzy logic algorithm classifies the shapes and determines the flight parameters, such as the speed, angle of attack and roll angle. Three different algorithms (homography, Iterative Closest Point (ICP), and Horns Absolute Orientation) were used to determine the pose estimation based on the vision system. Those techniques were tested on the HD Life-web camera with a moving baseline before using a stereo camera to find a suitable baseline and distance. The relations between the optimal baseline and depth distance are discussed, and several experiments with different image noise levels are performed to determine noise levels influence on distance and pose estimate accuracy. Measurements and estimation errors are provided and compared using different methods. The Deflection-Detection-Vision-System (DDVS) consists 
of a stereo camera positioned at the back end of the wing structure that reads the deflection of chosen landmark locations on the flexible wing for each image instantaneously. The DDVS characteristics and dynamic parameters were tested in wind tunnel at speeds ranging from 11 to $31 \mathrm{~m} / \mathrm{s}$, angles of attack (AOA) from $-20^{\circ}$ to $20^{\circ}$ and roll angles from $-10^{\circ}$ to $10^{\circ}$.

Controlling an autonomous UAV with flexible wing can be difficult using classical methods. Fundamentally, the model is a highly nonlinear, and in such cases, intelligent control strategies offer more suitable and advanced approach to designing a controller for the nonlinear system. An autopilot controller based on an intelligent controller known as the Adaptive Neuro-Fuzzy Inference System (ANFIS) algorithm was developed, and it applies the neural networks and fuzzy logic features in hybrid control architecture. To achieve optimal performance, three ANFIS modules were designed to control the altitude, heading angle and speed of the flexible wing UAV. The longitudinal motion controller and the inner loop (pitch rate feedback) of the longitudinal system are designed first, then a pitch tracker with an ANFIS controller is developed. The design of the altitude and speed controllers is related to the guidance and control system (outer loop controller) using the ANFIS controller design. The ANFIS controller performance is compared and evaluated with the ProportionalIntegral-Derivative (PID) controller. The lateral motion control is performed by an inner loop controller, that includes roll rate feedback, and the roll tracker is done with ANFIS controller. The heading direction controller based on ANFIS was added to supplement the guidance and control of the unmanned aerial vehicles. The proposed ANFIS controller was chosen because it has better performance than the classic controller. The controllers performance was verified using experimental data, as well as, extensive computer simulation. An inertial sensor was used and compared with DDVS for a flexible wing. In this work, the data fusion algorithm was applied to estimate 
the attitude for pitch and roll angles. The fusion algorithm based on Adaptive Unscented Kalman Filter (AUKF) was integrating measurements from an accelerometer sensor and DDVS. This method is prevented from diverging by an adaptive procedure based on fuzzy logic that modifies the noise characteristics and tuning factors of the weighted covariance. The results obtained from experiments and simulation show that, the performance of AUKF is better than that of regular UKF. Also it show high performance in both disturbance rejection and robustness against sensors noise. 


\section{Acknowledgments}

I would like to thank all of the people who encouraged and supported me during the undertaking of this research. To begin, I would like to express my gratitude to my Ph.D. program advisor, Professor Jurek Z. Sasiadek, for his continued support and guidance. He provided advice, opportunities, and assistance that greatly enhanced my research experience.

Many thanks to my mother, my father, and my brothers for the patience and the support in all my choices, no matter what they were. In particular, I thank my lovely wife and my children "Fatima, Mohammed Ridha and Zainab" for their company and understanding during this thesis activity.

Last but not certainly least, I would also like to thank my friends and fellow students for the great time spent together and the experiences shared. 


\section{Table of Contents}

$\begin{array}{lll}\text { Abstract } & \text { ii }\end{array}$

Acknowledgments $\quad$ v

Table of Contents $\quad$ vi

List of Tables $\quad x$

List of Figures $\quad$ xi

Nomenclature $\quad$ xvi

1 Introduction 1

1.1 Power Hang Glider . . . . . . . . . . . . . . . . . . . . . 1

1.2 Motivation ......................... 6

1.3 Contributions and Publication $\ldots \ldots \ldots \ldots \ldots$

1.4 Thesis Outline $\ldots \ldots \ldots \ldots \ldots \ldots$

2 Literature Review 10

2.1 Computer Vision for UAV . . . . . . . . . . . . . . . 10

2.2 Classification of The Wing Shape . . . . . . . . . . . . . 11

2.3 Adaptive Unscented Kalman filter (AUKF) . . . . . . . . . . . 12

2.4 Control (autopilot) $\ldots \ldots \ldots \ldots \ldots \ldots$ 
3 Machine Vision $\quad 15$

3.1 Introduction . . . . . . . . . . . . . . . . . 15

3.2 Stereo Vision Camera . . . . . . . . . . . . . . . . . . . . . . 15

3.3 Feature Detection and Tracking . . . . . . . . . . . . . . . . . 19

3.4 RANdom SAmple Consensus (RANSAC) . . . . . . . . . . . . . 20

3.5 Homography . . . . . . . . . . . . . . . . . . . . . . . 21

3.6 Homography Estimation . . . . . . . . . . . . . . . . . . . 24

3.7 Decomposition of Homography Matrix . . . . . . . . . . . . 25

3.7.1 Fast Homography Decomposition Method . . . . . . . . . 25

3.7.2 Iterative Closest Point Algorithm (ICP) . . . . . . . . . . . . 27

3.7.3 Horns Absolute Orientation Method . . . . . . . . . . . . 29

4 Equations of motion $\quad 31$

4.1 Introduction . . . . . . . . . . . . . . . . . . 31

4.2 Mathematical Modeling . . . . . . . . . . . . . . . . 31

4.2.1 The Forces and Moments of The Gravity . . . . . . . . . . . . 33

4.2.2 The Aerodynamic Forces and Moments . . . . . . . . . . . . . 34

4.2.3 Longitudinal Control . . . . . . . . . . . . . . . . . . . 37

4.2.4 Lateral Control . . . . . . . . . . . . . . . . . . . . . . 38

4.2.5 Total Aerodynamics With Thrust Force . . . . . . . . . . . . . 39

4.3 Equation of Motion Equilibrium . . . . . . . . . . . . . . . 40

5 Control System Design 43

5.1 Introduction . . . . . . . . . . . . . . . . 43

5.2 Longitudinal Control . . . . . . . . . . . . . . . . . . . 44

5.2.1 Inner Loop Pitch Attitude Tracker Design . . . . . . . . . . . 44

5.2.2 Outer Loop Altitude Hold Controller . . . . . . . . . . . . . . 45 
5.2.3 Airspeed Hold Controller . . . . . . . . . . . . . . . . . . . . . 46

5.3 Lateral Control . . . . . . . . . . . . . . . . . . . . 46

5.3.1 Inner Loops Roll Angle Hold Controller . . . . . . . . . . . . . 46

5.3.2 Heading Hold Controller . . . . . . . . . . . . . . . . . . . . . 47

5.4 Controllability and Observability . . . . . . . . . . . . 48

5.5 PID Controller . . . . . . . . . . . . . . . . . . . . . . . 49

5.6 ANFIS Controller . . . . . . . . . . . . . . . 51

5.7 Hybrid Learning Algorithm . . . . . . . . . . . . . . 53

6 Sensor Fusion $\quad 54$

6.1 Introduction . . . . . . . . . . . . . . . . . 54

6.2 Attitude Determination . . . . . . . . . . . . . . 54

6.3 Accelerometer . . . . . . . . . . . . . . . 56

6.4 Sensor Fusion . . . . . . . . . . . . . . . . . . . . 58

6.5 Adaptive Kalman Filter $(\mathrm{AUKF})$. . . . . . . . . . . . . . . . 59

6.5.1 Unscented Kalman Filter . . . . . . . . . . . . . . 60

6.5.2 Fuzzy Logic Adaptive System (FLAS) . . . . . . . . . . . . 63

7 Experimental Setup and Results $\quad 65$

7.1 Introduction . . . . . . . . . . . . . . . . 65

7.2 Machine Vision . . . . . . . . . . . . . . . . 65

7.2.1 Camera Calibration ............... 67

7.2.2 The Error Functions . . . . . . . . . . . . . . . . . . 69

7.2.3 The Pose Measurements . . . . . . . . . . . . . . . . 70

7.3 Results of the Flexible Wing . . . . . . . . . . . . . . . . . . . 74

7.3.1 Wing Shape . . . . . . . . . . . . . . . 75

7.3.2 Feature Extraction ................ . . 81 
7.3.3 Extract Deflection using the Stereo Camera . . . . . . . . . . 81

7.3.4 Classifying Wing Shapes with Fuzzy Logic . . . . . . . . . . 91

7.3.5 Test and Validation . . . . . . . . . . . . . . . . 95

7.4 Sensor Fusion . . . . . . . . . . . . . . . . . . . . . 99

7.5 Autopilot . . . . . . . . . . . . . . . . 105

7.5.1 Longitudinal Autopilot Simulation . . . . . . . . . . . . 105

7.5.2 Lateral Autopilot Simulation . . . . . . . . . . . . . . . . 116

7.6 Future Works . . . . . . . . . . . . . . . . . 120

8 Conclusion $\quad 121$

$\begin{array}{ll}\text { List of References } & 132\end{array}$

Appendix A Equivalent Aerodynamic Stability Derivatives 133 


\section{List of Tables}

6.1 Accelerometer noise density from manufacture . . . . . . . . . 58

7.1 Specification of Microsoft LifeCam HD-3000 . . . . . . . . . . . 66

7.2 Estimation of mobile robot position for all algorithms . . . . . . . . 72

7.3 Estimation of mobile robot position for two baselines . . . . . . . 73

7.4 Estimation of flying robot position for two baselines . . . . . . . . 73

7.5 Test Results for AOA . . . . . . . . . . . . . . . . . 86

7.6 Test Results for Roll angle . . . . . . . . . . . . . . . . . . . 87

7.7 Nodes deflection over the both sides. . . . . . . . . . . . . . . . 92

7.8 Fuzzy rules for the flexible wing at a positive deflection . . . . . . . 93

7.9 Fuzzy rules for the flexible wing at a negative deflection. . . . . . . . 93

7.10 Experimental results for attitude angles . . . . . . . . . . . . . 97

7.11 The Fuzzy Rule . . . . . . . . . . . . . . . . . . . . . . 100

7.12 Time domain analysis of pitch angle . . . . . . . . . . . . . 110

7.13 Time domain analysis of speed . . . . . . . . . . . . . . . 110

7.14 Time domain analysis of roll angle . . . . . . . . . . . . . . 117

7.15 Time domain analysis of heading angle . . . . . . . . . . . . 118

A.1 The longitudenal derivatives for the wing . . . . . . . . . . . 134

A.2 The lateral derivatives for the wing . . . . . . . . . . . . 135 


\section{List of Figures}

1.1 A typical ultralight trike with a Profi-TL wing [2] . . . . . . . . . 2

1.2 A side view of the engine and its propeller [2] . . . . . . . . . . 2

1.3 High performance wing $[2] \ldots \ldots \ldots$. . . . . . . . . . . 3

1.4 The wing structure beneath the canvas $[2] \ldots \ldots$. . . . . . . 3

1.5 Reference frames for wing, tricycle, and the main center of gravity . . 4

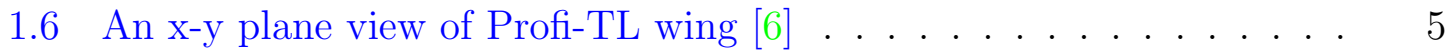

1.7 Flexible wing UAV . . . . . . . . . . . . . . . 5

3.1 StereoLabs ZED camera . . . . . . . . . . . . . . 16

3.2 Stereo camera modeling . . . . . . . . . . . . . . 16

3.3 Mathematical model of a stereo vision . . . . . . . . . . . . . . 19

3.4 The geometry of a homography mapping . . . . . . . . . . . . . 22

4.1 Longitudinal geometry . . . . . . . . . . . . . . . . . . . . 32

4.2 Lateral geometry . . . . . . . . . . . . . . . . . . 32

4.3 Longitudinal geometry . . . . . . . . . . . . . . . . . 38

4.4 Lateral geometry . . . . . . . . . . . . . . . . . . 39

5.11 Longitudinal Guidance and Control System for pitch hold . . . . . 45

5.2 Longitudinal Guidance and Control System for altitude hold . . . . . 45

5.3 Longitudinal Guidance and Control System for speed hold . . . . . . 46

5.4 Lateral Guidance and Control System for roll hold . . . . . . . . . . . 47 
5.5 Lateral Guidance and Control System for heading hold . . . . . . . . 47

5.6 Block Diagram of PID Controller [76] . . . . . . . . . . . 50

5.7 TSK fuzzy model $[83] \ldots \ldots \ldots$. . . . . . . . . . . . . 51

5.8 Adaptive neuro fuzzy interference system structure . . . . . . . . 53

6.1 ADXL 330 accelerometer sensor . . . . . . . . . . . . . 57

6.2 Arduino Uno R3 board . . . . . . . . . . . . . . 58

6.3 Block diagram of AUKF . . . . . . . . . . . . . . . . . . 59

6.4 The feedback control system for the flexible wing UAV . . . . . . . 59

6.5 The input and output of FLAS . . . . . . . . . . . . . . 64

7.1 Camera calibration toolbox for MATLAB with a model chessboard

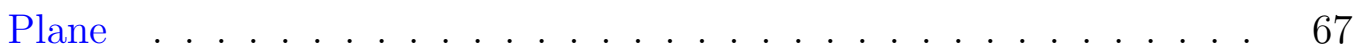

7.2 Camera extrinsic parameters . . . . . . . . . . . . . 68

7.3 Plot of the log error against the noise levels . . . . . . . . . . . 70

7.4 Relative translation error via noise level . . . . . . . . . . . . . 70

7.5 Initial and desired targets from front view . . . . . . . . . . . 71

7.6 Initial and desired targets from top view . . . . . . . . . . . . 71

7.7 The Detection-Deflection Vision System(DDVS) for the flexible wing

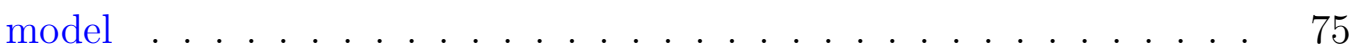

7.8 Back view of the flexible wing in wind tunnel . . . . . . . . . . 76

7.9 Front view of the flexible wing in wind tunnel . . . . . . . . . . 76

7.10 Features position . . . . . . . . . . . . . . . 77

7.11 Shape of wing at $\mathrm{V}=31 \mathrm{~m} / \mathrm{s} \ldots \ldots \ldots$. . . . . . . . . 77

7.12 Shape of wing at $\mathrm{V}=11 \mathrm{~m} / \mathrm{s}$ and $\mathrm{AOA}=+20^{\circ} \ldots \ldots \ldots$

7.13 Shape of wing at $\mathrm{V}=21 \mathrm{~m} / \mathrm{s}$ and $\mathrm{AOA}=+20^{0} \ldots \ldots \ldots$

7.14 Shape of wing at $\mathrm{V}=31 \mathrm{~m} / \mathrm{s}$ and $\mathrm{AOA}=+20^{0} \ldots \ldots$. . . . . . 79

7.15 Shape of wing at $\mathrm{V}=31 \mathrm{~m} / \mathrm{s}$ and $\mathrm{AOA}=-20^{0} \ldots \ldots$. . . . . . 79 
7.16 Shape of wing at $\mathrm{V}=31 \mathrm{~m} / \mathrm{s}$, Roll angle $=10^{\circ}$ and $\mathrm{AOA}=+20^{\circ} \ldots$. . . 80

7.17 Shape of wing at $\mathrm{V}=11 \mathrm{~m} / \mathrm{s}$, Roll angle $=-10^{\circ}$ and $\mathrm{AOA}=-20^{\circ} \ldots$. . 80

7.18 The matching of the feature points . . . . . . . . . . . 81

7.19 Shape of wing at $\mathrm{AOA}=0^{0}$ and roll angle $=0^{0} \quad \ldots \ldots$. . . . . . . 82

7.20 Shape of wing at $\mathrm{AOA}=-20^{0}$ and roll angle $=0^{0} \ldots \ldots$. . . . . . 83

7.21 Shape of wing at $\mathrm{AOA}=0^{0}$ and roll angle $=-10^{0} \ldots \ldots$. . . . . 83

7.22 Shape of wing at $\mathrm{AOA}=-20^{0}$ and roll angle $=-10^{0} \ldots$. . . . . . . 84

7.23 Shape of wing at $\mathrm{AOA}=20^{0}$ and roll angle $=-10^{0} \ldots \ldots$. . . . . . 84

7.24 Shape of wing at $\mathrm{AOA}=20^{0}$ and roll angle $=0^{0} \ldots \ldots$. . . . . 85

7.25 Tracking points along the experimental tests . . . . . . . . . 88

7.26 Maximum deflection vs. AOA . . . . . . . . . . . . . . 89

7.27 Maximum deflection vs. speed at $\mathrm{AOA}=-20$ deg. . . . . . . . . . . . 90

7.28 Maximum deflection vs. speed at $\mathrm{AOA}=0$ deg. . . . . . . . . . . 90

7.29 Maximum deflection vs. speed at $\mathrm{AOA}=20$ deg. . . . . . . . . . . 91

7.30 Block diagram of Fuzzy logic . . . . . . . . . . . . . . . . . . . 92

7.31 Membership function of the input node $1 \ldots$. . . . . . . . . . 93

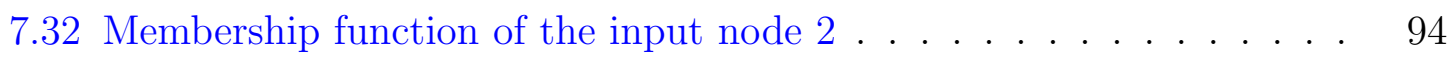

7.33 Membership function of the output wind speed . . . . . . . . . . . . 94

7.34 Membership function of the output AOA . . . . . . . . . . . . 94

7.35 Membership function of the output roll angle . . . . . . . . . . . 95

7.36 Two sensor are fixed in flexible wing . . . . . . . . . . . . 96

7.37 Accelerometer sensor with Arduino board . . . . . . . . . . . . 96

7.38 Pitch error for the proposed DDVS, accelerometer and AUKF . . . . 98

7.39 Roll error for the proposed DDVS, accelerometer and AUKF . . . . . 98

7.40 Covariance membership function of input variable . . . . . . . . . . . 101

7.41 Mean value membership function of input variable . . . . . . . . . 101 
7.42 Membership function of output variable . . . . . . . . . . . . . . 101

7.43 Pitch angle estimation obtained from the UKF and the AUKF . . . . 103

7.44 Roll angle estimation obtained from the UKF and the AUKF . . . . . 103

7.45 The error estimation of pitch angle . . . . . . . . . . . . 104

7.46 The error estimation of roll angle . . . . . . . . . . . . . . . 104

7.47 Input variable "error" for pitch angle . . . . . . . . . . . . . . . . 106

7.48 Input variable "error change" for pitch angle . . . . . . . . . . . . . . 106

7.49 Output surface of fuzzy modules for pitch angle . . . . . . . . . . . . 107

7.50 Input variable "error" for altitude control . . . . . . . . . . . . . . 107

7.51 Input variable "error change" for altitude control . . . . . . . . . . . 107

7.52 Output surface of fuzzy modules for altitude control . . . . . . . . . 108

7.53 Input variable "error" for speed control . . . . . . . . . . . . . . . . . 108

7.54 Input variable "error change" for speed control . . . . . . . . . . . . . 108

7.55 Output surface of fuzzy modules for speed control . . . . . . . . . . . 109

7.56 Pitch-hold longitudinal autopilot response . . . . . . . . . . . . . . 110

7.57 Altitude-hold longitudinal autopilot response . . . . . . . . . . . . . . 111

7.58 Speed-hold longitudinal autopilot response . . . . . . . . . . . . . . . 111

7.59 ANFIS pitch controller for tracking different signal input . . . . . . . 112

7.60 ANFIS altitude controller for tracking different altitudese . . . . . . . 113

7.61 ANFIS speed controller for tracking different speeds . . . . . . . . . 113

7.62 The effect of disturbance on pitch tracker . . . . . . . . . . . . . 114

7.63 The effect of disturbance on altitude . . . . . . . . . . . . . . 115

7.64 The effect of disturbance on speed . . . . . . . . . . . . . . . 115

7.65 Roll controller for tracking different angles . . . . . . . . . . . . . . . 117

7.66 Heading controller for tracking different angles . . . . . . . . . . . 118

7.67 The effect of disturbance on roll angle . . . . . . . . . . . . . . . 119 
7.68 The effect of disturbance on heading . . . . . . . . . . . . . . . . . 119 


\section{Nomenclature}

- For machine vision in Chapter 3

$b$ the baseline

d the disparity

d the distance between the origin camera to the plane

$\left(f_{x}, f_{y}\right) \quad$ the focal length in $\mathrm{x}$ and $\mathrm{y}$ directions

H Homography matrix

$I_{x x}, I_{y y}, I_{x y}$ the convolution of the second-order image derivatives computed

$k$ an intrinsic camera matrix

$\left(m, m^{*}\right)$ the normalized Euclidean coordinate vector

n the surface normal vector

$\left(p, p^{*}\right) \quad$ the pixel coordinate vector

$P_{A}, P_{B} \quad$ frames $\mathrm{A}$ and $\mathrm{B}$

$(\mathrm{p}, \mathrm{x}) \quad$ two frames

$\mathrm{R}$ rotation matrix

s a scale vector

t translation vector

$\mathrm{T}$ translation vector

$(\mathrm{u}, \mathrm{v})$ pixel point

$u_{o}, v_{0}$ the principal point in term of pixel dimension 
$(\mathrm{X}, \mathrm{Y}, \mathrm{Z})$ coordinate system

$\alpha_{i} \quad$ the scalar depth ratio

$\beta \quad$ the skew coefficient

$\mu \quad$ center of mass

$\sum$ the cross-covariance

Subscripts

cl the left camera

cr the right camera

1 left

r right

w world

- For Kalman filter in Chapter 6

$A(k)$ the transition matrix

$B(k)$ the control matrix

$H(k)$ the measurement matrix

$K_{k} \quad$ Kalman filter gain

$P_{x} \quad$ a covariance

$P_{v v} \quad$ innovation covariance

$P_{x y} \quad$ cross covariance

Q system noise covariance matrix

$\mathrm{R}$ observation noise covariance matrix

$u(k) \quad$ the input vector

$v(k)$ the measurement noise

$v$ mean of residuals

$x(k)$ the state vector 
$\mathrm{X}$ the sigma points

$w(k)$ the process noise

W weighted points

$z(k)$ the measurement vector

$\alpha$ the softening factor

$\beta \quad$ the Gaussian factor

$\lambda$ the scaling parameter

$\mu \quad$ the first parameter for DOD

$\xi \quad$ the second parameter for DOD

- For the dynamic model in Chapter 4

b wing span (m)

c local cord $(\mathrm{m})$

$\bar{c} \quad \frac{2}{S} \int_{0}^{\frac{b}{2}} c^{2} d y=$ mean aerodynamic cord $(\mathrm{m})$

$c_{g} \quad$ center of gravity

$C_{D} \quad \frac{D}{\frac{1}{2} \rho S V^{2}}=$ drag Coefficient

$C_{L} \quad \frac{L}{\frac{1}{2} \rho S V^{2}}=$ lift Coefficient

$C_{l \beta}, C_{n \beta} \quad$ sideslip derivatives

$C_{l p}, C_{n p} \quad$ wing rolling derivatives

$C_{l r}, C_{n r} \quad$ wing yawing derivatives

$C_{l} \quad \frac{L}{\frac{1}{2} \rho b S V^{2}}=$ rolling-moment coefficient

$\bar{C}_{m} \quad \frac{M}{\frac{1}{2} \rho S \bar{c} V^{2}}=$ pitching moment Coefficient

$C_{m q} \quad$ wing pitching derivative

$C_{T} \quad \frac{T}{\frac{1}{2} \rho S V^{2}}=$ thrust Coefficient 
$C_{n} \quad \frac{N}{\frac{1}{2} \rho b S V^{2}}=$ yawing-moment coefficient

$C_{w o} \quad \frac{m g}{\frac{1}{2} \rho S u_{o}^{2}}=$ yawing-moment coefficient

$C_{x} \quad C_{T}+C_{L} \alpha_{x}-C_{D}$

$C_{z} \quad-\left(C_{L}+C_{D} \alpha_{x}\right)$

D $\operatorname{Drag}(\mathrm{N})$

$I_{x} \quad \int y^{2}+z^{2} d m=$ moment of inertia about $\mathrm{x}$-axis $\left(\mathrm{kg} \cdot \mathrm{m}^{2}\right)$

Iy $\int x^{2}+z^{2} d m=$ moment of inertia about y-axis $\left(\mathrm{kg} \cdot \mathrm{m}^{2}\right)$

$I_{z} \quad \int y^{2}+x^{2} d m=$ moment of inertia about z-axis $\left(\mathrm{kg} \cdot \mathrm{m}^{2}\right)$

$I_{y z}=I_{z y} \quad \int y z d m=$ product of Inertia $\left(\mathrm{kg} \cdot \mathrm{m}^{2}\right)$

$I_{x z}=I_{x y} \quad \int x z d m=$ product of Inertia $\left(\mathrm{kg} \cdot \mathrm{m}^{2}\right)$

$I_{y x}=I_{x y} \quad \int y x d m=$ product of Inertia $\left(\mathrm{kg} \cdot \mathrm{m}^{2}\right)$

$\mathrm{L} \quad \operatorname{lift}(\mathrm{N})$

L rolling moment (N.m)

m mass $(\mathrm{kg})$

M pitching moment (N.m)

$\mathrm{N} \quad$ yawing moment (N.m)

$\mathrm{p}$ roll rate $(\mathrm{rad} / \mathrm{s})$

q pitch rate $(\mathrm{rad} / \mathrm{s})$

r yaw rate $(\mathrm{rad} / \mathrm{s})$

$\mathrm{S} \quad$ wing area $\left(\mathrm{m}^{2}\right)$

u axial velocity $(\mathrm{m} / \mathrm{s})$

v lateral velocity $(\mathrm{m} / \mathrm{s})$

$V_{e} \quad$ reference equilibrium airspeed $(\mathrm{m} / \mathrm{s})$

w normal velocity $(\mathrm{m} / \mathrm{s})$

$\mathrm{T}$ thrust $(\mathrm{N})$

$(\mathrm{X}, \mathrm{Y}, \mathrm{Z}) \quad$ component of resultant aerodynamic force $(\mathrm{N})$ 
$\alpha \quad$ angle of attach(AOA) (radians)

$\beta \quad$ sideslip angle (radians)

$\delta \quad$ longitudinal control angle (radians)

$\delta_{t h} \quad$ thrust Control

$\rho \quad$ air density $\left(\mathrm{kg} / \mathrm{m}^{3}\right)$

$(\theta, \phi, \psi) \quad$ euler angles (radians)

$\xi \quad$ lateral control angle (radians)

Subscripts

a aerodynamic

c carriage or tricycle

e equilibrium

o the reference flight condition

w wing 


\section{ABBREVIATION DEFINITIONS}

ANFIS Adaptive Neuro-Fuzzy Inference System

AOA Angle of attack

AUKF Adaptive Unscented Kalman Filter

DDVS Deflection - Detection Vision System

DLT Direct Linear Transform

DOD Degree of Divergence

EKF Extended Kalman Filter

FLAS Fuzzy Logic Adaptive System

ICP Iterative Closest Point

PID Proportional - Integral - Derivative

RANSAC RANdom SAmple Consensus

SURF Speeded Up Robust Features

UKF Unscented Kalman Filter

UT Unscented Transformation 


\section{Chapter 1}

\section{Introduction}

\subsection{Power Hang Glider}

Powered hang gliders, also known as ultralight trikes, flex-wing trikes, microlight trikes, or microlights, have attracted great attention in the air sports community since the 1980s. Recently, commercial companies and the military have shown interest in them as they are relatively affordable and have low maintenance and performance costs. These aerial vehicles can carry the pilot and one passengers, and are equipped with simple flight instruments for navigation. The ultralight trike structure and flight control principles are similar to non-motorized hang gliders, previously invented and patented by Francis Rogallo in 1948 [1]. However, ultralight trikes have a fuselage usually the shape of a trike that is suspended from the airframe and at the wing's center of gravity, as shown in Fig. 1.1.

A propeller is connected to an engine at the back of the vehicle to create thrust, as illustrated in Fig. 1.2. Thus, the pilot does not need to launch downhill as it is possible to take-off from level ground. Compared to a hang glider, the high-performance wing of an ultralight trike is to larger, with a stronger composite frame of cables, tubes, and canvas to maintain the wing configuration and handle the extra weight, as shown 
in Fig. 1.3 and 1.4.

A regular fixed-wing aircraft is controlled [2] by the pilot, who can control the motion about the $\mathrm{x}, \mathrm{y}$ and $\mathrm{z}$ axes during the flight. Longitudinal rotation (angular displacement about the $\mathrm{x}$ axis (roll) is the primary task of ailerons on the outer rear of each wing that can move up and down, while the rotation about the y axis (pitch) is governed by the lift wing (elevator) on the horizontal tail surface tilting up or down.

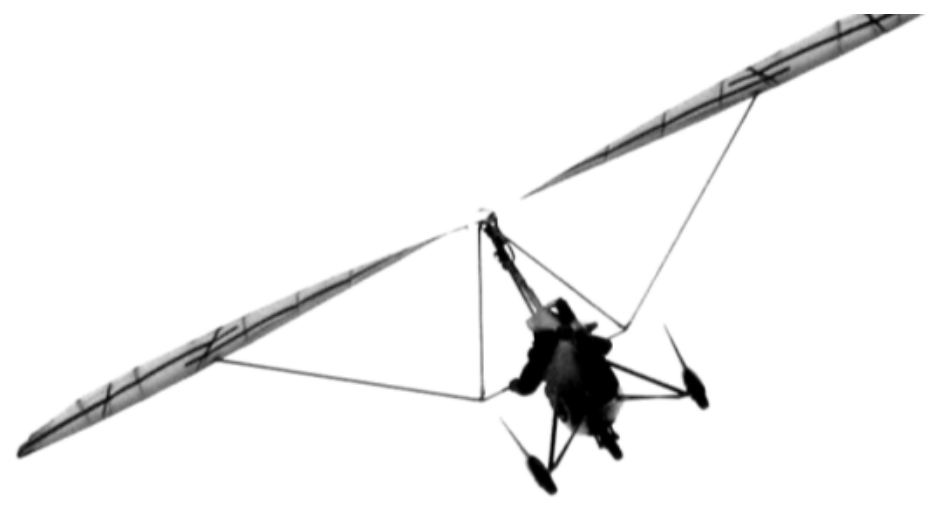

Figure 1.1: A typical ultralight trike with a Profi-TL wing [2]

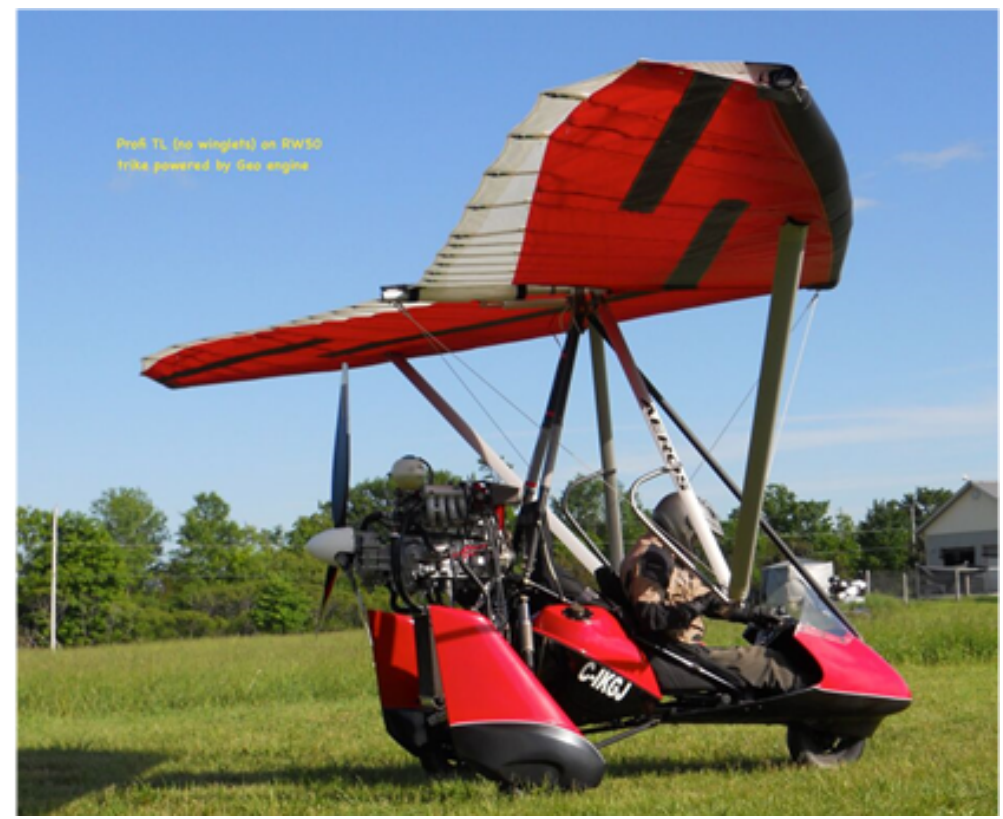

Figure 1.2: A side view of the engine and its propeller [2] 
Vertical rotation around the $\mathrm{z}$ axis (yaw) is controlled by tunning the vertical tail fin (rudder) side to side [3]. The control principles for an ultralight trike are quite different than those of fixed-wing aircraft, since an ultralight trike has no ailerons, elevators or rudders connected to the flight control mechanisms. It is controlled by simple weight shifts applied by the pilot (Fig. 1.5).

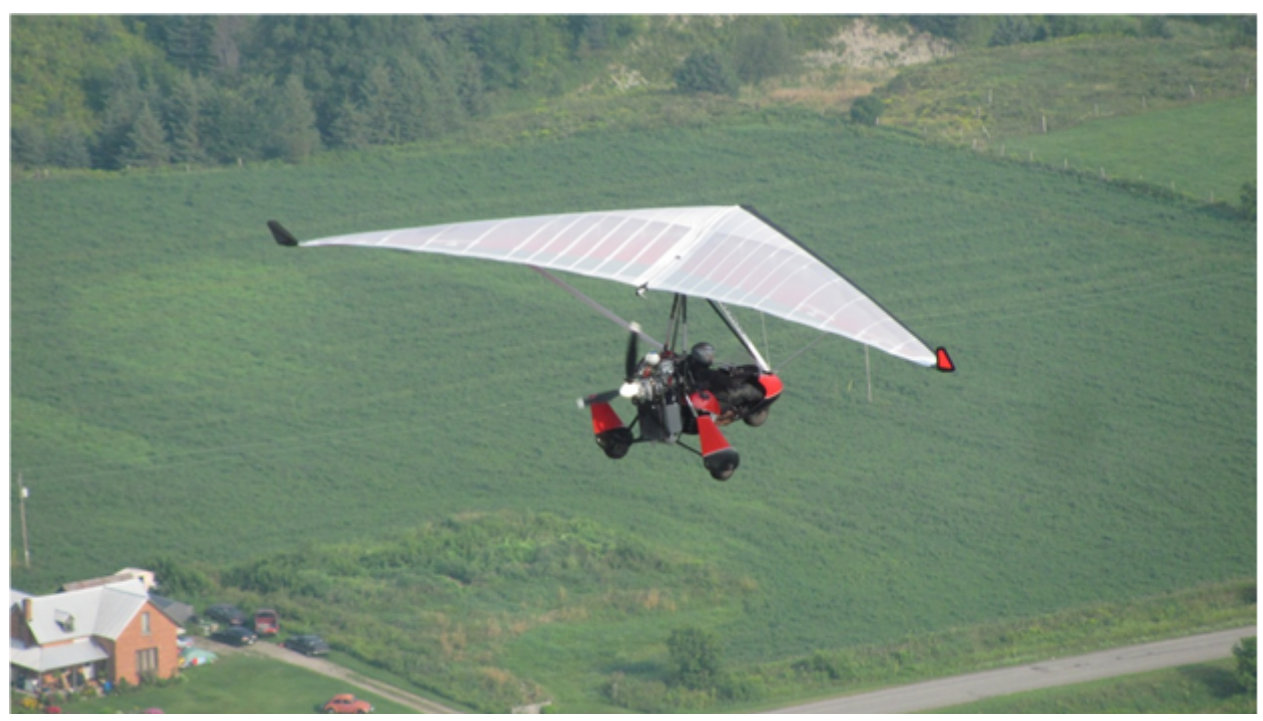

Figure 1.3: High performance wing [2]

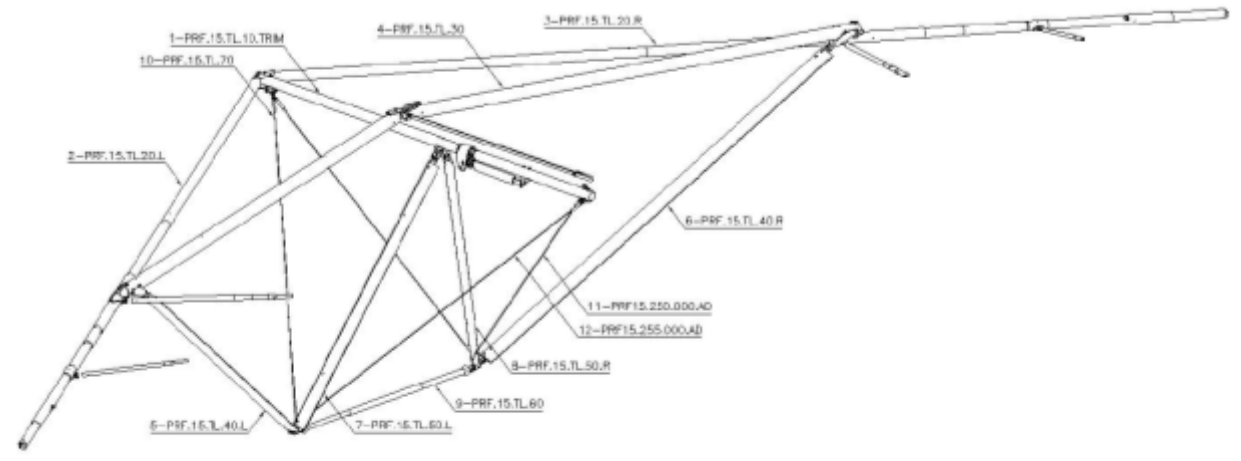

Figure 1.4: The wing structure beneath the canvas [2] 


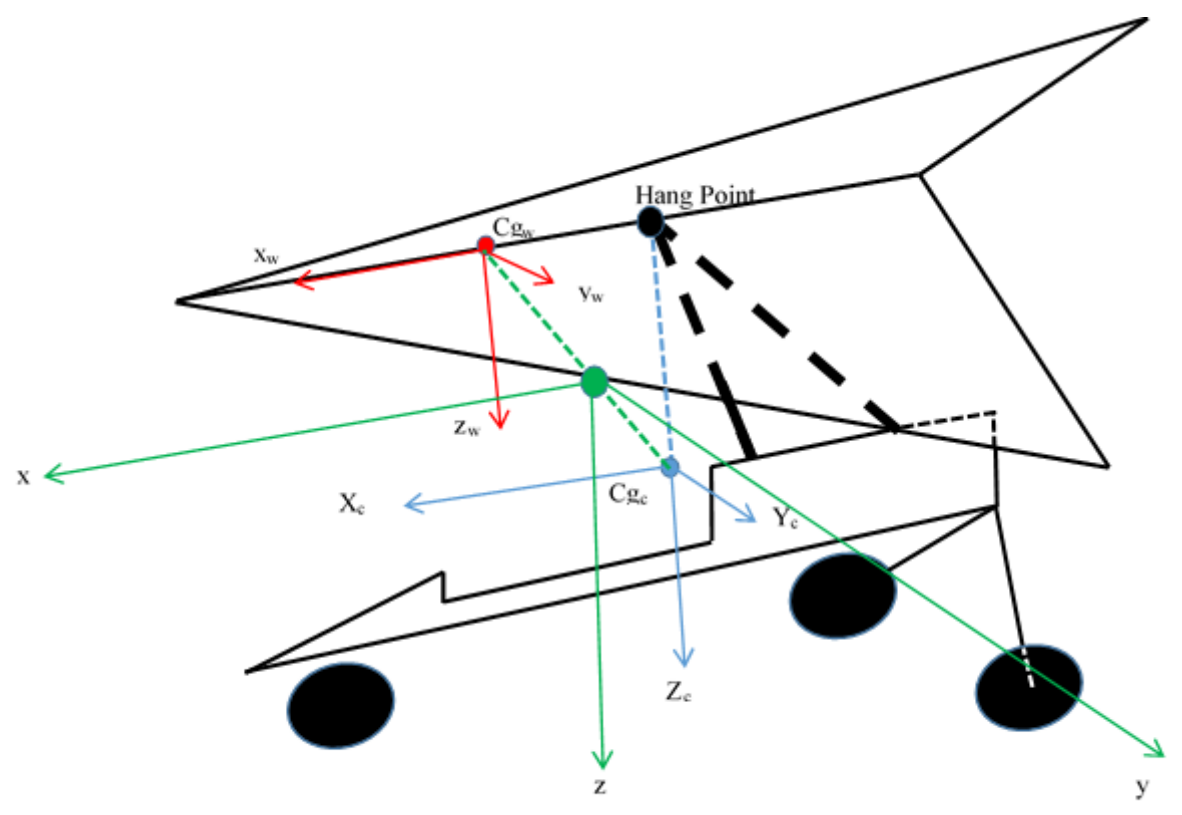

Figure 1.5: Reference frames for wing, tricycle, and the main center of gravity

In the proposed model, the weight of the fuselage is the sum of weights of pilot, engine, seats, onboard instruments, fuel tank, propeller, and other devices or equipment attached to the fuselage. For example, moving the control bar to the left shifts the weight of the fuselage to the right and the center of gravity to the left, which changes the aerodynamic moment of the vehicle. Likewise, when the pilot moves the control bar to the right, the total suspending weight is shifted to the left. The sideto-side movement of the control bar allows the vehicle to perform roll task, that is, to rotate around the $\mathrm{x}$ axis. There are also responses when the control bar is shifted forward or backward, as this causes the vehicle to pitch (i.e. rotate about y axis) when taking-off, landing or changing altitude during flight [4].

There is no yaw control in the flight control mechanism, so to return to equilibrium the pilot must rely on the natural stability of the wing when simultaneously moving the control bar side-to-side and forward-to-backward [5]. Deriving the motion 


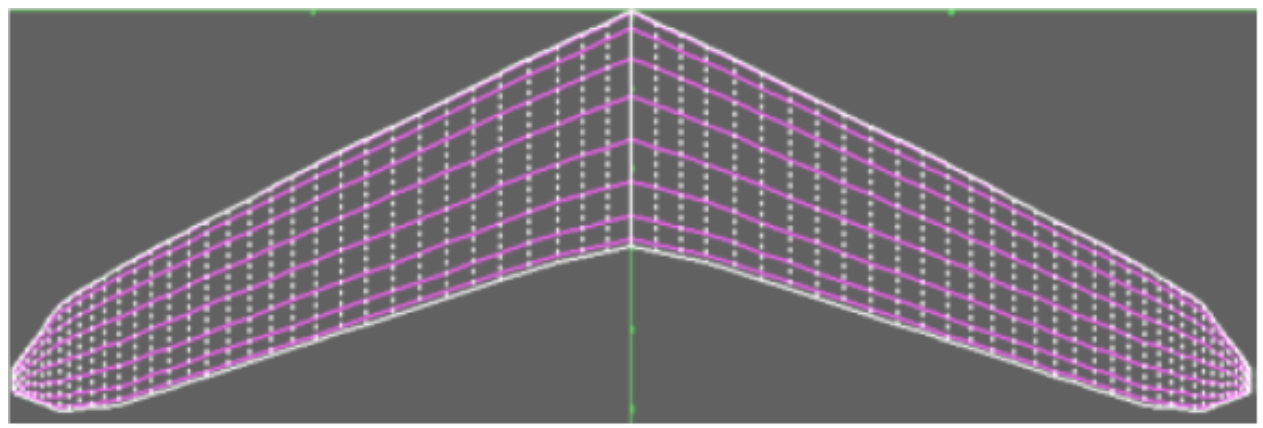

Figure 1.6: An $x-y$ plane view of Profi-TL wing [6]

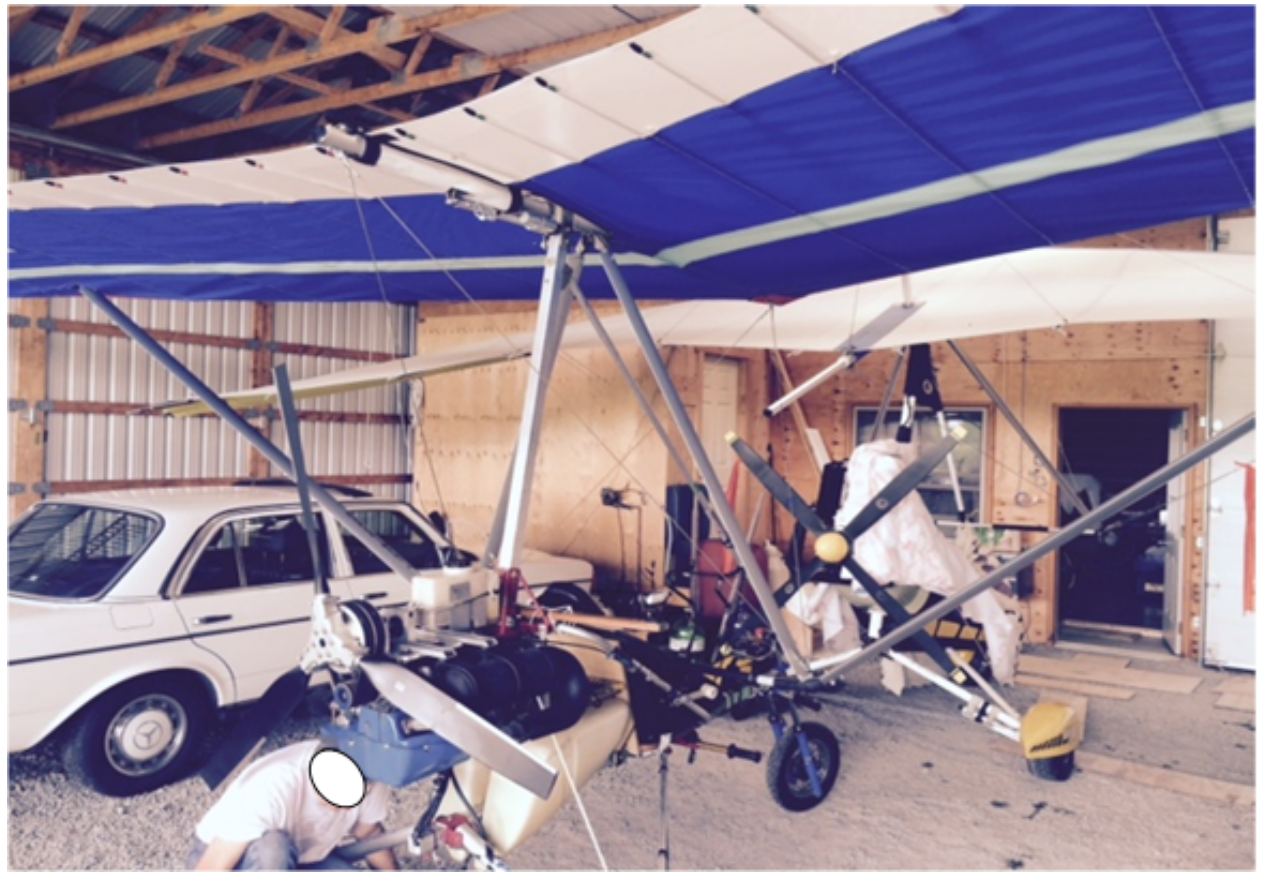

Figure 1.7: Flexible wing UAV

equations is a key step in following a systematic approach to design a control motion algorithm for unmanned ultralight trike control in flight, which is the ultimate goal of this project. At this point, however, the main focus will be to develop the kinetics of this aerial vehicle, and derive the equilibrium governing equations of motion for an ultralight trike with a Profi $T L$ wing and a hand-made trike, as illustrated in Fig. 1.6 and $1.7[6]$. 


\subsection{Motivation}

The primary aim of this thesis is to develop and implement a method or system that can visually guide and control a UAV. The extensive information that collected by a camera can be used for accurate high performance UAV control, navigation, guidance and tracking, and this data can be applied to the feedback of an aircraft's performance and behavior. The performance requirements to control a power hang glider vary as functions of the flight conditions (e.g. speed, altitude) and pilot commands. Intelligent control for a flexible wing UAV based on Deflection-Detection-Vision-System (DDVS) has been demonstrated, and it involves four different steps. First, a stereo camera identifies the shape of the wing; second, the wing shape is classify by fuzzy logic; third, the design, implementation and testing is conducted on the longitudinal and lateral autopilots for various stages of the flight; and finally, the sensor fusion is adapted to calculate attitude estimation by integrating the reference data collected from DDVS and the measurement data from the accelerometer, based on the Adaptive Unscented Kalman Filter (AUKF). This algorithm is designing to optimize the robust proposal method (DDVS) for pitch and roll angles.

\subsection{Contributions and Publication}

The main contributions of this thesis are summarized below:

1. Development of the Deflection-Detection-Vision-System (DDVS) that can be controlled from a platform using machine vision only. The algorithm provides a method to control the flexible wing, depending on identification the wing shape.

2. The three-dimensional (3D) pose estimation based on the stereo camera has been proposed and validated. The 3D point for an object (landmark) with 
respect to the camera coordinate system (the relative position and attitude of the UAV with respect to landmarks) is obtained by using three methods (a fast Homography decomposition technique, Iterative Closest Point (ICP) and Horns Absolute Orientation).

3. A novel approach for determining wing shape and classification by employing machine vision and fuzzy logic to create a simple tool. This approach is a system that measures the wing deflection based on DDVS. Fuzzy logic is used to classify the wing shape and optimize particular flight performance criteria.

4. In this work, the longitudinal and lateral autopilots are implemented based on the Adaptive Neuro-Fuzzy Inference System (ANFIS) controller trained to learn and predict the responses of the UAV from the history of response and control signals.

5. A sensor fusion has been designed to verify the DDVS method, and to estimate the attitude angles using the Adaptive Unscented Kalman Filter (AUKF). 
The publication related to this thesis work is listed below:

- Malik M. A. Al-Isawi, and J. Z. Sasiadek, Pose Estimation for Mobile Robot via Visual Navigation, Proceedings of the CARO3 3rd Conference on Aerospace Robotics, November 2015 and accepted for publication in Aerospace Robotics III, published by Springer Publishers.

- Malik M. A. Al-Isawi, and J. Z. Sasiadek, Navigation and Control of a Space Robot Capturing Moving Target, Proceedings of the RoMoCo17 The 11 International Workshop on Robot Motion and Control, 3-5 July 2017.

- Malik M. A. Al-Isawi, and J. Z. Sasiadek, Control of Flexible Wing UAV Using Stereo Camera, accepted for publication in Aerospace Robotics III, published by Springer Publishers.

- Malik M. A. Al-Isawi, and J. Z. Sasiadek, Guidance and Control of Autonomous, Flexible Wing UAV with Advanced Vision System, Proceeding of IEEE MMAR Conference in 2018.

- Malik M. A. Al-Isawi, and J. Z. Sasiadek, Guidance and Control of a Robot Capturing an Uncooperative Space Target, Journal of Intelligent and Robotic Systems (JIRS) 2018. 


\subsection{Thesis Outline}

Chapter 2 presents a literature review of the computer vision, classification, adaptive Kalman filter and autopilot design.

Chapter 3 reviews the computer vision processing algorithms used for visual matching and tracking. It also explains how these algorithms are employed for the robust projective transformations estimation used to obtain the pose estimation.

Chapter 4 presents the modeling of hang glider based on the Newton-Euler formalism.

Chapter 5 discusses the longitudinal and lateral control system for a UAV, and explains the ANFIS controller.

Chapter 6 presents a sensor fusion to estimate and integrate different types of data using AUKF.

Chapter 7 highlights the experimental tests and results using a single camera and a stereo camera. It shows results for a novel system DDVS that classifies the wing shape to obtain the state vector of the UAV. An autopilot for both longitudinal and lateral motion control is designed and tested. Finally, sensor fusion integration for DDVS/ accelerometers data are shown and applied for the attitude control of UAV. Future work are suggested.

Chapter 8 includes the conclusions . 


\section{Chapter 2}

\section{Literature Review}

\subsection{Computer Vision for UAV}

Computer vision has proven to be a powerful and low-cost sensor that is useful for many applications in robotics and control systems, and new appriaches are continually beging developed that reduce cost, size and weight, while increasing the capacity to acquire and process high-resolution images with adequate frequency for use in control systems on mobile and flying robotics. Several recent studies have focused on pose estimation or shape determination for many applications. The vision-based pose estimation technique evaluates and calculates relative translations and rotations of two images for the same target. The authors in [7] and [8] propose methods to reconstruct the cameras pose with respect to an object based on an accurate geometric model of the object. The pose estimation from homography and the investigation of measurement errors are shown in [9], and the general homography decomposition to evaluate robotic motion was used by [10]. Faugeras and Lustman [11] described a more specific decomposition of the homography when a robot is moving on a planar surface. Both approaches provide two possible solutions for the camera pose. A new algorithm for relative pose estimation between two images based on new decomposition was 
proposed in [12]. The Iterative Closest Point (ICP) algorithm was used in [13] to estimate position and attitude based on 3D data, and the mathematical preliminaries of computing the closest point were shown in [14]. The performance was achieved with three different focal lengths. A closed-form solution for three or more points using unit quaternions was presented in [15]. The pose estimation for UAV pose estimation is shown in [16]. This algorithm allows accurate navigation when a GPS is absent, by using a stereo camera in UAV. The authors in [17] developed a new algorithm based on Extended Kalman Filter (EKF) for Pose estimation, and apllied it to aerial UAV refueling in serious turbulence. A new algorithm for the Minimaum number of points Linear pose estimation with known Zenith direction (MLZ) was presented by [18]. This algorithm estimated the pose of a camera with a known gravity vector.

\subsection{Classification of The Wing Shape}

The Fuzzy Logic control (FLC) has conducted some limited research regarding classifing deflections and control of a flexible wing using a vision system. Some of the work involved aerodynamic coefficients and the aeroelasticity of flying, and the authors in [19] focused on static aeroelasticity of flying wings. A theoretical study of dynamic response estimation of a highly flexible flying-wing are described in [20]. Aerodynamic coefficients and structural displacements from wind tunnel tests are presented in [21], and wing flexibility on a Micro Air Vehicles (MAV) is also investigated. The videogrammetry technique presented in [22] studied the deformation and attitude of static and dynamic wings in a wind-tunnel model, and non-contact videogrammetry techniques measured the dynamic behavior of flexible mini-UAV wings in wind tunnel tests [23]. The authors in [24] proposed a wing twist technique based on camera photogrammetrics, studies structure deformation and collected measurement data in 
flight tests using digital image correlation [25]. Static aeroelasticity was also measured and discussed by [26]. In addition, the uncertainty of videogrammetric techniques was applied to wind tunnel models, and fuzzy logic implemented by [27] was used to find the surface deflections of a flexible wing to achieve the desired roll angle.

\subsection{Adaptive Unscented Kalman filter (AUKF)}

Many researchers focused their efforts on guidance and navigation aspects, and a navigation technique based on a visual servoing system combined with a six degree of freedom (DOF) robotic manipulator was used to capture a moving target [28]. A novel concept to identify a moving object and its trajectory design in space was presented in [29], [30], and [31], and focused on capturing an autonomous satellite using a visual servoing system. The pose of a large non-cooperative target was determined in [32] by using two cameras to facilitate recognition of a selected rectangular feature object.

The Unscented Kalman filter (UKF) based on a neural network (NN) algorithm was developed in [33], and these were integrated to estimate nonlinear states to enhance a nonlinear dynamic model. A novel adaptive UKF (AUKF) was developed by [34], and applied to nonlinear joint estimation for time-varying states and parameters. This algorithm was based on a cost function that determined the error between the covariance matrices of innovation and their corresponding estimates. The authors in [35]presented a new robust adaptive UKF that corrects the process noise covariance or measurement noise covariance, depending on the type of fault. The algorithm was applied to the attitude estimate of a pico satellite, and the orientation estimate of aircraft and UAV based on the AUKF presented in [36]. The proposed filter estimates the acceleration generated over regular cruising flights, and can predict the roll and pitch orientation angles with reasonable accuracy. 


\subsection{Control (autopilot)}

Today, UAVs are becoming more useful due to their advantages for civil and military applications. Different control techniques have been successfully developed and verified for UAV flight control systems, including optimal control, predictive control, adaptive control and robust control [37], [38]. The most popular techniques has been a proportional-integral-derivative controller (PID), and analytical methods are used to tune the conventional structure of PID controller [39]. The author in [40] presents a new approach to control, stabilize and disturbance rejection of the attitude subsystem of a quadrotor. A self-tuned PID is used to design the controller of an autopilot based on the longitudinal for altitude and speed and lateral motion for heading angle of an Aerosonde UAV.

The fuzzy logic approach has the potential to compensate for the effects of nonlinearity and uncertainty, and it provides strong robustness independent of the mathematical model of a system. In addition, designing a fuzzy logic controller is fast and simple and it is easily maintained because the rules can be understood and explained by human experts [41]. Autonomous UAV such as helicopters have been the most popular robotic platforms, as they are easy to control for vertical take-off and landing, as well as for stable flight [42], [43]. In [44], a stable gain-scheduling PID controller based on the grid point concept was developed for nonlinear systems. The autopilot controller provided more accurate tracking paths and robustness with respect to environmental disturbances, particularly wind. Small UAVs are highly sensitive to wind [45]. The fuzzy PID controller was developed to improve the longitudinal motion (pitch control) performance of the aircraft [46]. This paper focuses on how to combine the advantages of fuzzy control and PID control to improve the dynamic performance [47]. The longitudinal model of the Unmanned Aerial Vehicle (UAV) was 
established and controlled using fuzzy PID [48], and the control strategies included velocity in inner loop and height in outer loop. The authors in [49] compared different controllers used with the dynamic model of a quadcopter platform. The controllers were an ITAE tuned PID, a classic Linear - Quadratic Regulator (LQR) and a PID tuned with an LQR loop. The conventional LQR method presented in [50] efficiently solved the problems of fixed-wing UAVs being disturbed by air current that reduce flight stability. Longitudinal control for a flying wing UAV based on LQR allowed it to achieve satisfactory longitudinal flying performance [51]. An Adaptive NeuroFuzzy Inference System (ANFIS) controller was develoed to control the position of the UAV in 3D by adjusting the pitch and roll angles, and the throttle position. The ANFIS algorithm allows UAV to accomplish their mission autonomously [52]. Lateral controller develope by [53] used ANFIS to enhance performance, as ANFIS can approximate nonlinear functions, and doesnt need an accurate nonlinear model. ANFIS modules were designed and developed autonomous controllers to regulate the airspeed, heading angle and altitude of a fixed-wing UAV in longitudinal directional motion [54]. A direct heuristic dynamic programming (HDP) method using ANFIS was designed to control a hypersonic vehicle, and ANFIS was superior than neural networks with one-hidden-layer architectures, particularly when dealing with parametric uncertainties [55]. UAV performance was improved by using ANFIS techniques to a model of a low-cost vision UAV [56]. 


\section{Chapter 3}

\section{Machine Vision}

\subsection{Introduction}

This chapter introduces most of the image processing techniques for a variety of applications. First is a description of how pinhole and stereo cameras are modeled. Then feature detection and tracking methods are examined to determine the relevant interest feature points useful for identifying and tracking regions of interest in image sequences. Finally, different approaches to determine 3D pose are proposed.

\subsection{Stereo Vision Camera}

Stereo vision is a process to extract the depth of a 3-D scene from different points. A stereo camera is acually two cameras that are horizontally aligned, with a fixed distance known as the baseline. The ZED stereo camera as shown in Fig. 3.1 was selected to provide both vision and depth data, as can capture high-resolution sideby-side video on USB3. Furthermore, this camera is already calibrated has known intrinsic and extrinsic parameters, and its epipolar constraint can be used to reduce the time to search corresponding points in two images by searching only one line 


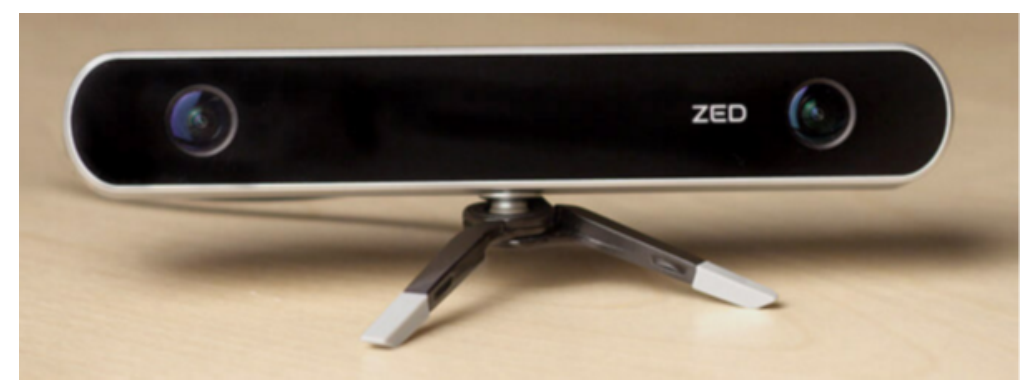

Figure 3.1: StereoLabs ZED camera

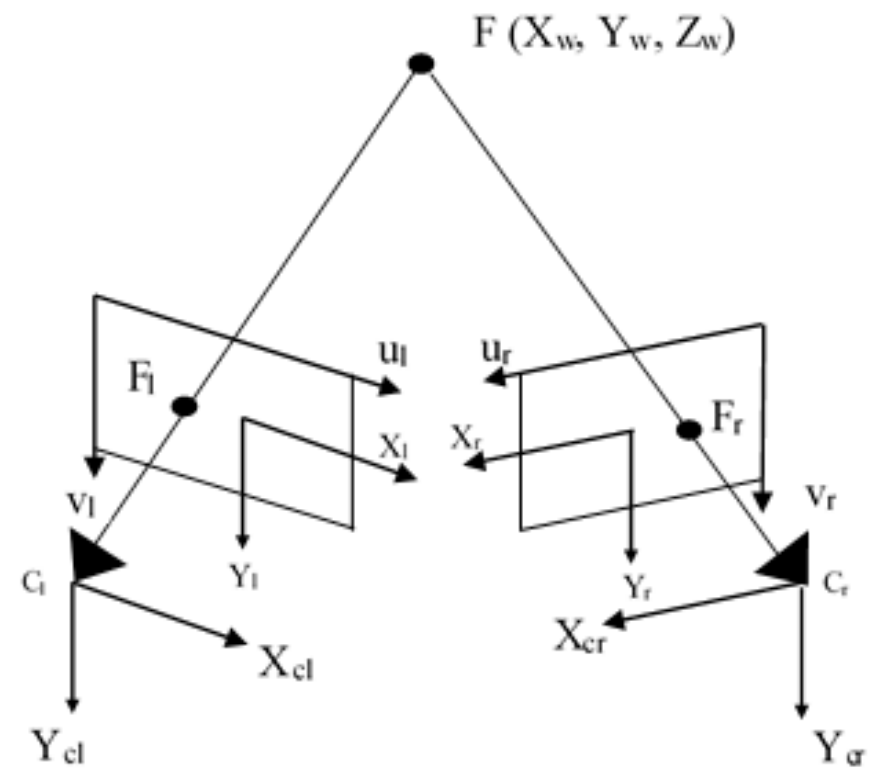

Figure 3.2: Stereo camera modeling

in the image rather than the entire image [57]. This method can be computed by rectifying the homography between images so their epipolar lines are parallel and aligned, thereby making the search for corresponding points highly efficient. The mathematical model of stereo vision is as follows: Assume a feature point, F has the corresponding pixel points as the scene, $F_{l}$ is the image formed on the left camera $C_{l}$ and $F_{r}$ is the image formed on the right camera $C_{r}$ as shown in Fig. 3.2. The projection matrix can be given as: 
- For the left camera

$$
Z_{l}\left[\begin{array}{l}
u_{l} \\
v_{l} \\
1
\end{array}\right]=\left[\begin{array}{lll}
f_{x} & \beta & u_{0} \\
0 & f_{y} & v_{0} \\
0 & 0 & 1
\end{array}\right]_{l}\left[\begin{array}{cccc}
1 & 0 & 0 & 0 \\
0 & 1 & 0 & 0 \\
0 & 0 & 1 & 0
\end{array}\right]\left[\begin{array}{ll}
R & T \\
0 & 1
\end{array}\right]_{l}\left[\begin{array}{c}
X \\
Y \\
Z \\
1
\end{array}\right]_{w}
$$

or

$$
Z_{l}\left[\begin{array}{l}
u_{l} \\
v_{l} \\
1
\end{array}\right]=\left[\begin{array}{cccc}
n_{11}^{l} & n_{12}^{l} & n_{13}^{l} & n_{14}^{l} \\
n_{21}^{l} & n_{22}^{l} & n_{23}^{l} & n_{24}^{l} \\
n_{31}^{l} & n_{32}^{l} & n_{33}^{l} & n_{34}^{l}
\end{array}\right]\left[\begin{array}{c}
X \\
Y \\
Z \\
1
\end{array}\right]_{w}
$$

Rearrange eq. 3.2

$$
\left[\begin{array}{ccc}
u_{l} n_{31}^{l}-n_{11}^{l} & u_{l} n_{32}^{l}-n_{12}^{l} & u_{l} n_{33}^{l}-n_{13}^{l} \\
v_{l} n_{31}^{l}-n_{21}^{l} & v_{l} n_{32}^{l}-n_{22}^{l} & v_{l} n_{33}^{l}-n_{23}^{l}
\end{array}\right]\left[\begin{array}{l}
X \\
Y \\
Z
\end{array}\right]=\left[\begin{array}{l}
-u_{l} n_{34}^{l}+n_{14}^{l} \\
-v_{l} n_{34}^{l}+n_{24}^{l}
\end{array}\right]
$$


- For the right camera

$$
Z_{r}\left[\begin{array}{l}
u_{r} \\
v_{r} \\
1
\end{array}\right]=\left[\begin{array}{lll}
f_{x} & \beta & u_{0} \\
0 & f_{y} & v_{0} \\
0 & 0 & 1
\end{array}\right]_{r}\left[\begin{array}{llll}
1 & 0 & 0 & 0 \\
0 & 1 & 0 & 0 \\
0 & 0 & 1 & 0
\end{array}\right]\left[\begin{array}{ll}
R & T \\
0 & 1
\end{array}\right]_{r}\left[\begin{array}{l}
X \\
Y \\
Z \\
1
\end{array}\right]_{w}
$$

or

$$
Z_{r}\left[\begin{array}{l}
u_{r} \\
v_{r} \\
1
\end{array}\right]=\left[\begin{array}{cccc}
n_{11}^{r} & n_{12}^{r} & n_{13}^{r} & n_{14}^{r} \\
n_{21}^{r} & n_{22}^{r} & n_{23}^{r} & n_{24}^{r} \\
n_{31}^{r} & n_{32}^{r} & n_{33}^{r} & n_{34}^{r}
\end{array}\right]\left[\begin{array}{l}
X \\
Y \\
Z \\
1
\end{array}\right]_{w}
$$

Rearrange eq. 3.5

$$
\left[\begin{array}{ccc}
u_{r} n_{31}^{r}-n_{11}^{r} & u_{r} n_{32}^{r}-n_{12}^{r} & u_{r} n_{33}^{r}-n_{13}^{r} \\
v_{r} n_{31}^{r}-n_{21}^{r} & v_{r} n_{32}^{r}-n_{22}^{r} & v_{r} n_{33}^{r}-n_{23}^{r}
\end{array}\right]\left[\begin{array}{l}
X \\
Y \\
Z
\end{array}\right]=\left[\begin{array}{l}
-u_{r} n_{34}^{r}+n_{14}^{r} \\
-v_{r} n_{34}^{r}+n_{24}^{r}
\end{array}\right]
$$

where $Z_{l}$ and $Z_{r}$ are depth of left and right camera with respect to target.

The world coordinate system in Equation's 3.3 and 3.6 can be calculated with the least square method. The corresponding pixel points of $\mathrm{F}$ in the left and right images are at the same height, depending on the epipolar constraint as shown in Fig. 3.3. The depth equation can be derived based on the geometry of similar triangles, 


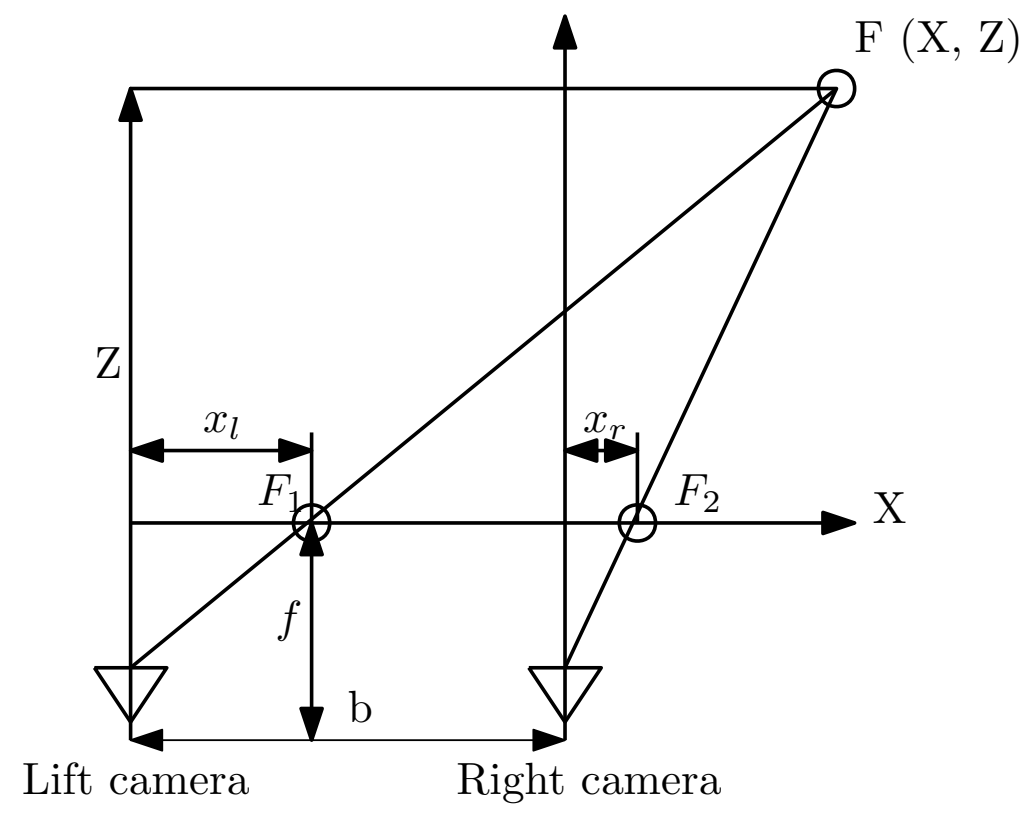

Figure 3.3: Mathematical model of a stereo vision

$$
Z=\frac{f b}{\left(x_{l}-x_{r}\right)}=\frac{f b}{d}
$$

where $\mathrm{b}$ is the baseline, $f$ is a focal length and $d$ is the disparity. As the baseline and focal length of the stereo camera are known, the distance $\mathrm{Z}$ in the scene can be calculated based on the disparity between the two images. The corresponding points $F_{l}$ and $F_{r}$ are related by the homography matrix [58], [59].

\subsection{Feature Detection and Tracking}

Feature point detection is the first step in machine vision. It finds interest feature points in each frame, whereas the tracking of feature points refers to the corresponding of features from frame to frame [60]. Therefore, it is important to extract features from an image, such as corners, blobs and edges. The Speeded Up Robust Features (SURF) detection method developed by Bay et al. [61] is based on a Hessian matrix, 
and since this matrix has robustness and computation speed method, it can be used to analyze local image structures. It is expressed in the form [62]:-

$$
H=\left[\begin{array}{cc}
I_{x x} & I_{x y} \\
I_{x y} & I_{y y}
\end{array}\right]
$$

where $I_{x x}, I_{x y}$, and $I_{y y}$ are the convolution of the second-order image derivatives computed using the Gaussian function. To detect interest features, it searches for a subset of points where the derivative responses are high in two orthogonal directions, that is, the detector finds points where the determinant of the Hessian matrix has a local maximum.

\subsection{RANdom SAmple Consensus (RANSAC)}

RANSAC is an iterative method to estimate parameters of a mathematical model from a set of observed data which has outliers. It was introduced by Fischler and Bolles [63] using a set of data with a large number of outliers. According to [64] RANSAC is the most commonly used robust estimation method for homography. The idea behind the algorithm is simple. For several iterations, a random sample of four correspondences is selected, and the homography $(\mathrm{H})$ is computed from them. All other correspondences are classified as inliers or outliers, depending on their concurrence with H. After the iterations are complete the iteration with the largest number of inliers is selected. $\mathrm{H}$ can then be recomputed from all the correspondence inliers in that iteration.

The RANSAC method can be summarized as:

1. Initialize the number of estimation, threshold and maximum inliers. 
2. Calculate the number of iterations using the following equation [63], [65].

$$
T=\frac{\log \epsilon}{\log (1-q)}
$$

where: $\epsilon$ is the probability of incorrect identification of the model, and $q$ is calculated based on the following equation:

$$
q=\left(\frac{N_{i}}{N}\right)^{k}
$$

where $N_{i}$ is the number of points that belong to the consensus set, $\mathrm{N}$ is the total number of points, and $\mathrm{k}$ is the minimal number of data necessary to clearly define the model.

3. Randomly select four feature pairs.

4. Check if these points are collinear, if so go to step 3.

5. Compute the homography $\mathrm{H}$.

6. Compute the Euclidean distance between two points d $\left(\mathrm{H} p_{i}, p_{i}^{*}\right)$

7. If $\mathrm{d} \leq$ the threshold, then select the pairs that agree with the homography and record all the inliers.

8. Repeat steps 3 to 7 until the number of iteration has been reached.

9. Re-compute the $\mathrm{H}$ estimate using all the inliers.

\subsection{Homography}

Assume the camera is moving in the $x_{i} y_{i}$ plane and $\mathrm{z}$ axis is orthogonal to $x_{i} y_{i}$. The camera views four or more planar and non-collinear feature points lying in a plane in 


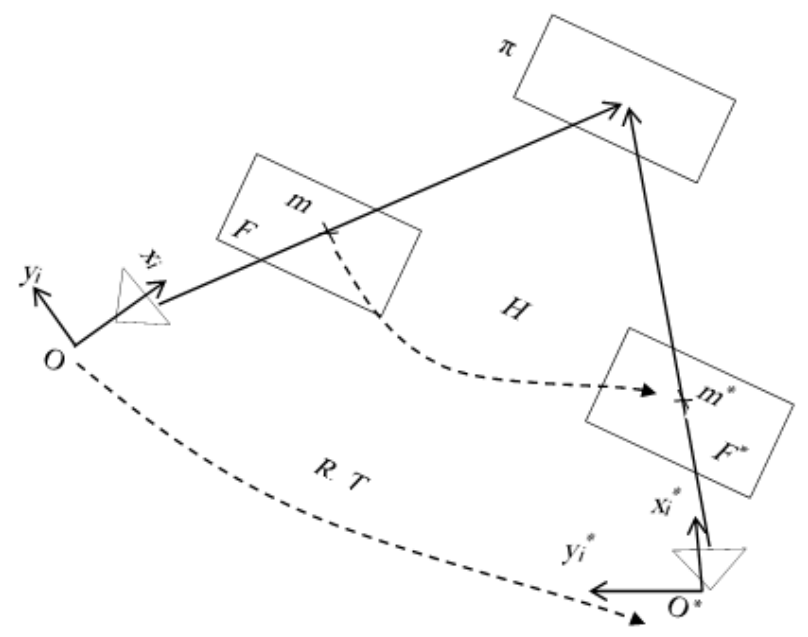

Figure 3.4: The geometry of a homography mapping

front of the camera. The points expressed in frames $\left(F\right.$ and $\left.F^{*}\right)$ are denoted by $\left(x_{i}\right.$ $\left.y_{i} z_{i}\right)$ and $\left(x_{i}^{*} y_{i}^{*} z_{i}^{*}\right) \in \mathrm{R}$ respectively [66], and the normalized Euclidean coordinate vector can be expressed by $m$ and $m^{*} \in R^{3}$.

$$
m=\left[\frac{x_{i}}{z_{i}}, \frac{y_{i}}{z_{i}}, 1\right]^{T} \text { and } \quad m^{*}=\left[\frac{x_{i}^{*}}{z_{i}^{*}}, \frac{y_{i}^{*}}{z_{i}^{*}}, 1\right]^{T}
$$

As shown in Fig 3.4,the feature points $m$ and $m^{*}$ in frames $\left(F\right.$ and $\left.F^{*}\right)$ respectively are related by the homography matrix $\mathrm{H} \in R^{3 x 3}$ [67] as:

$$
m=\alpha_{i} H m^{*}
$$

where $\alpha_{i}=\frac{z_{i}^{*}}{z_{i}}$, is the scalar depth ratio. From the projective geometry, the Euclidean coordinates for $\left(m\right.$ and $\left.m^{*}\right)$ can be expressed as image coordinates (pixel coordinates) as following:-

$$
p=k m \text { and } p^{*}=k m^{*}
$$


where $k \in R^{3 x 3}$ is intrinsic camera matrix that can be determined by camera calibration $[11]:-$

$$
k=\left[\begin{array}{lll}
f_{x} & \beta & u_{0} \\
0 & f_{y} & v_{0} \\
0 & 0 & 1
\end{array}\right]
$$

where $\left(f_{x}, f_{y}\right)$ : are the focal lengths in the $\mathrm{x}$ and $\mathrm{y}$ directions, respectively. $\left(u_{0}\right.$ and $v_{0}$ ): are the coordinates of the principal point in terms of pixel dimensions. $\beta$ is the skew coefficient between the cameras $\mathrm{x}$ and $\mathrm{y}$ axes, this is often zero. $\left(p_{i}\right.$ and $\left.p_{i}^{*}\right)$ : are pixel coordinates of two images denoted by:

$$
p=\left[u_{i}, v_{i}, 1\right]^{T} \quad \text { and } \quad p^{*}=\left[u_{i}^{*}, v_{i}^{*}, 1\right]^{T}
$$

Using (3.13) in relation (3.12), we get

$$
\begin{gathered}
p_{i}=\alpha_{i} k H k^{-1} p_{i}^{*}=\alpha_{i} G p_{i}^{*} \\
H=k^{-1} G k
\end{gathered}
$$




\subsection{Homography Estimation}

The pixel coordinates $\left(p_{i}\right.$ and $\left.p_{i}^{*}\right)$ for the two images denoted in Equation (3.15) and can be represented as [68], [69]:

$$
\begin{gathered}
{\left[\begin{array}{c}
u_{i}^{*} \\
v_{i}^{*} \\
1
\end{array}\right]=\left[\begin{array}{lll}
G_{11} & G_{12} & G_{13} \\
G_{21} & G_{22} & G_{23} \\
G_{31} & G_{32} & G_{33}
\end{array}\right]\left[\begin{array}{l}
u_{i} \\
v_{i} \\
1
\end{array}\right]} \\
u_{i}^{*}=\frac{G_{11} u_{i}+G_{12} v_{i}+G_{13}}{G_{31} u_{i}+G_{32} v_{i}+G_{33}} \\
v_{i}^{*}=\frac{G_{21} u_{i}+G_{22} v_{i}+G_{23}}{G_{31} u_{i}+G_{32} v_{i}+G_{33}}
\end{gathered}
$$

Rearranging the equation above gives:-

$$
A_{i} G=0
$$

where $\mathrm{G}=\left(G_{11}, G_{12}, G_{13}, G_{21}, G_{22}, G_{23}, G_{31}, G_{32}, G_{33}\right)^{T}$ and,

$$
A_{i}=\left[\begin{array}{lllllllll}
u_{i} & v_{i} & 1 & 0 & 0 & 0 & -u_{i} u_{i}^{*} & -v_{i} u_{i}^{*} & -u_{i}^{*} \\
0 & 0 & 0 & u_{i} & v_{i} & 1 & -u_{i} v_{i}^{*} & -v_{i} v_{i}^{*} & -v_{i}^{*}
\end{array}\right]
$$

Since the homography matrix has eight degrees of freedom, at least four points on a plane are required to determine the homography up to a scale factor by solving (3.21) using the linear least square method. 


\subsection{Decomposition of Homography Matrix}

The aim of this section is to extract information from the environment using the camera image, then determine the rotation and translation vectors from $\mathrm{O}$ to $O^{*}$ with the homography matrix. There are several methods to decompose a homography matrix, and three different algorithms were used to explore the direct method for the vision system: fast Homography decomposition technique, Iterative Closest Point (ICP) and Horns Absolute Orientation. The Faugeras decomposition method is one of the most common methods [11].

\subsubsection{Fast Homography Decomposition Method}

The homography matrix $\mathrm{H}$ can be decomposed as:

$$
H=R-\frac{t}{d} n^{T}
$$

where $\mathrm{H} \in R^{3 \times 3}$ is the homography matrix, and $\mathrm{R} \in R^{3 \times 3}$ and $\mathrm{t} \in R^{3}$ are the rotation matrix and the translation vector from $O$ to $O^{*}$ respectively. $\mathrm{d} \in R$ is the distance between the origin camera and the plane $\pi$, and $\mathrm{n} \in R^{3}$ is the surface normal vector to plane $\pi$ as in [11], [67]: According to the planner motion of the camera, the rotation $\mathrm{R}$ and translation $\mathrm{t}$ can be written as follows:

$$
R=\left[\begin{array}{ccc}
\cos (\theta) & -\sin (\theta) & 0 \\
\sin (\theta) & \cos (\theta) & 0 \\
0 & 0 & 1
\end{array}\right]
$$




$$
T=\frac{t}{d}=\frac{1}{d}\left[\begin{array}{lll}
t_{x} & t_{y} & 0
\end{array}\right]^{T}=\left[\begin{array}{lll}
T_{1} & T_{2} & 0
\end{array}\right]^{T}
$$

where $\theta \in R$ is the rotational angle around the $z_{i}$ axis from $O$ to $O^{*}, t_{x}$, and $t_{y}$ are the translate along $x_{i}$ and $y_{i}$ respectively, and $\mathrm{d}$ is the distance from the origin of $\mathrm{O}$ to the reference plane $\pi$. The $\mathrm{H}$ matrix can be calculated by substituting $(3.23$ and $3.24)$ into (3.22) [58]:

$$
H=\left[\begin{array}{ccc}
\cos (\theta)+T_{1} n_{1} & -\sin (\theta)+T_{1} n_{2} & T_{1} n_{3} \\
\sin (\theta)+T_{2} n_{1} & \cos (\theta)+T_{2} n_{2} & T_{2} n_{3} \\
0 & 0 & 1
\end{array}\right]=\left[\begin{array}{ccc}
h_{11} & h_{12} & h_{13} \\
h_{21} & h_{22} & h_{23} \\
0 & 0 & 1
\end{array}\right]
$$

It is clear from (3.25) that the algorithm for homography decomposition will be divided into three cases, depending on $h_{13}$ and $h_{23}$ [58].

1. In case $1 h_{13}^{2} \neq 0, h_{23}^{2} \neq 0$

$$
\begin{gathered}
T_{1}=\epsilon_{1} \sqrt{\frac{h_{13}^{2}\left[\left(h_{12}+h_{21}\right)^{2}+\left(h_{11}-h_{22}\right)^{2}\right]}{h_{13}^{2}+h_{23}^{2}}+h_{13}^{2}} \\
T_{2}=\epsilon_{1} \operatorname{sgn}\left(h_{23}, h_{13}\right) \sqrt{\frac{h_{23}^{2}\left[\left(h_{12}+h_{21}\right)^{2}+\left(h_{11}-h_{22}\right)^{2}\right]}{h_{13}^{2}+h_{23}^{2}}+h_{23}^{2}}
\end{gathered}
$$

where $\epsilon_{1}=\mp 1$, two solutions of $\mathrm{T}$ can be selected according to [55], and we can compute the $\mathrm{n}$ vector in (3.22) as follows:

$$
\begin{aligned}
& n_{1}=\frac{t_{1}\left(h_{11}-h_{22}\right)+t_{2}\left(h_{12}+h_{21}\right)}{t_{1}^{2}+t_{2}^{2}} \\
& n_{2}=\frac{t_{1}\left(h_{12}+h_{21}\right)-t_{2}\left(h_{11}-h_{22}\right)}{t_{1}^{2}+t_{2}^{2}}
\end{aligned}
$$




$$
n_{3}=\epsilon_{1} \operatorname{sgn}\left(h_{13}\right) \sqrt{\frac{h_{13}^{2}+h_{23}^{2}}{t_{1}^{2}+t_{2}^{2}}}
$$

It is quite easy to calculate the rotation matrix from (3.22).

2. In case $2 h_{13}^{2} \neq 0, h_{23}^{2}=0$ or $h_{13}^{2}=0, h_{23}^{2} \neq 0$

$$
\begin{gathered}
T_{1}=\epsilon_{1} \sqrt{\frac{h_{13}^{2}\left[\left(h_{12}+h_{21}\right)^{2}+\left(h_{11}-h_{22}\right)^{2}\right]}{h_{13}^{2}+h_{23}^{2}}+h_{13}^{2}} \\
T_{2}=0 \\
n_{1}=\frac{\left(h_{11}-h_{22}\right)}{t_{1}} \\
n_{2}=\frac{\left(h_{12}+h_{21}\right)}{t_{1}} \\
n_{3}=\frac{h_{13}}{t_{1}}
\end{gathered}
$$

we can also calculate the rotation matrix from (3.22).

3. In case $3 h_{13}^{2}=0$, and $h_{23}^{2}=0$

we have: if $h_{11}=h_{22}$, and $h_{21}=-h_{12}$ then $T_{1}=T_{2}=0$, and the homography degenerates to a rotation matrix. Otherwise, the decomposition degenerates to a simplified version of a 2 by 2 homography matrix. The Singular Value Decomposition (SVD) can be used to decomposite the $\mathrm{H}$ matrix using the techniques similar to the Faugeras algorithm [11].

\subsubsection{Iterative Closest Point Algorithm (ICP)}

An ICP algorithm attempts to match two sets of points, one of which might be a reference image and another a set of data points describing the ranges to certain 
points on an object. The ICP algorithm gives an estimate of camera translation and rotation between data taken from stereo images [13], [14]. The ICP algorithm is as follows:

A point set $\mathrm{p}$ of the source image point with $N_{p}$, and the destination points which become x with $N_{x}$ are given

1. Compute the centers of mass for $\mu_{p}$ and $\mu_{x}$ :

$$
\mu_{p}=\frac{1}{N_{p}} \sum_{i=1}^{N_{p}} p_{i}, \quad \mu_{x}=\frac{1}{N_{x}} \sum_{i=1}^{N_{x}} x_{i}
$$

2. The cross-covariance is:

$$
\left.\sum p x=\frac{1}{N_{p}} \sum_{i=1}^{N_{p}}\left[\left(p_{i}-\mu_{p}\right)\right]\left(x_{i}-\mu_{x}\right)^{T}\right]
$$

3. The rotation matrix $\mathrm{R}$ is given by Singular Value Decomposition (SVD) of the matrix $\sum p x$ :

$$
\sum p x=U S V^{T}, \text { and } \quad R=U V^{T}
$$

4. The translation $\mathrm{T}$ associated with the transformation is:

$$
T=\mu_{x}-R \mu_{p}
$$

5. Terminate the iteration when the change in mean-square error falls below the threshold:

$$
f(R, t)=\frac{1}{N_{p}} \sum_{i=1}^{N_{p}}\left\|x_{i}-R p_{i}-T\right\|^{2}
$$

6. Steps 1-5 are repeated until convergence is within the tolerance. 


\subsubsection{Horns Absolute Orientation Method}

The transformation between two Cartesian coordinate systems can be considered the result of a rigid-body motion, and can thus be decomposed into rotation and translation. If the coordinates of the four points in each frame are $P_{A, i}$ and $P_{B, i}$, the mathematical relationship between these sets of points is [15]:

$$
P_{A, i}=s R_{A / B} P_{B, i}+T_{A / B}
$$

where $\mathrm{s}$ is a scale vector and $R_{A / B}$ and $T_{A / B}$ are rotation and translation matrices between frames A and frame B. The absolute orientation algorithm is as follow:

- Subtract the centroid of the point sets for both frames.

$$
P_{A, i}^{*}=P_{A, i}-\sum_{j} P_{A, j}, \quad P_{B, i}^{*}=P_{B, i}-\sum_{j} P_{B, j}
$$

- The intermediate matrices:

$$
M=\left[\begin{array}{ccc}
S_{x x} & S_{x y} & S_{x z} \\
S_{y x} & S_{y y} & S_{y z} \\
S_{z x} & S_{z y} & S_{z z}
\end{array}\right]=\sum_{i} P_{A, i}^{*}\left(P_{B, i}^{*}\right)^{T}
$$


- and

$$
N=\left[\begin{array}{cccc}
S_{x x}+S_{y y}+S_{z z} & S_{y z}-S_{z y} & S_{z x}-S_{x z} & S_{x y}-S_{y x} \\
S_{y z}-S_{z y} & S_{x x}-S_{y y}-S_{z z} & S_{x y}+S_{y x} & S_{z x}+S_{x z} \\
S_{z x}-S_{x z} & S_{x y}+S_{y x} & -S_{x x}+S_{y y}-S_{z z} & S_{y z}+S_{z y} \\
S_{x y}-S_{y x} & S_{z x}+S_{x z} & S_{y z}+S_{z y} & -S_{x x}-S_{y y}+S_{z z}
\end{array}\right]
$$

- The rotation between frames $\mathrm{A}$ and $\mathrm{B}$ is the quaternion matrix $\mathrm{N}$ which is the eigenvector corresponding to the maximum eigenvalue of $\mathrm{N}$.

- The quaternion minimizes by the mean squared error function:

$$
f=\sum_{i}\left\|P_{A, i}-s R_{A / B} P_{B, i}-T_{A / B}\right\|^{2}
$$

- The scale can be found as:

$$
s=\frac{\sum_{i}\left\|P_{A, i}^{*}\right\|^{2}}{\sum_{i}\left\|P_{B, i}^{*}\right\|^{2}}
$$

- The translation vector can be found by solving the last remaining variable in Equation (3.41). 


\section{Chapter 4}

\section{Equations of motion}

\subsection{Introduction}

This chapter considers the mathematical models of a power hang glider (UAV). The model shown in Fig. 4.1 is a flexible multibody system that is subjected to gravity, aerodynamics, propulsion and control forces, and the bodies are broadly identified as the wing and the fuselage. There are two types of equations: kinematics and kinetics. Kinematics has to do with motion subjected to a general force, and kinetics examines the forces involved to produce motion. The equations of motion are linearized using perturbation theory, and the final results are state-space models of longitudinal and lateral motions.

\subsection{Mathematical Modeling}

The longitudinal geometry is shown in Fig. 4.1. an orthogonal fixed body axis system oxyz is defined to the vehicle $c g$. It lies on the line that connects the wing's center of gravity $\left(c g_{w}\right)$ that defined by the coordinates $\left(X_{w}, Y_{w}, Z_{w}\right)$ and the center of gravity of the carriage $\left(c g_{c}\right)$ is defined by coordinates $\left(x_{c}, y_{c}, z_{c}\right)$. In steady straight flight with 
velocity $\mathrm{V}$, the pitch attitude is $\theta$, angle of attack is $\alpha$ and flight path angle $\gamma$. The longitudinal control angle $\delta_{\text {long }}$ is defined as the angle between the hang strap and a line parallel to the $c g$. The axial force $\mathrm{X}$, normal force $\mathrm{Z}$, thrust force $\mathrm{T}$, and pitching moment $\mathrm{M}$ are shown in Fig. 4.1. The lateral geometry is shown in Fig. 4.2. However, the lateral control is $\delta_{\text {lat }}$. Also the lateral side force $\mathrm{Y}$, normal force $\mathrm{Z}$ and rolling moment L are shown in Fig. 4.2. The center of gravity changes when positioned on the line that connects the wing center of gravity and the fuselage center of gravity.

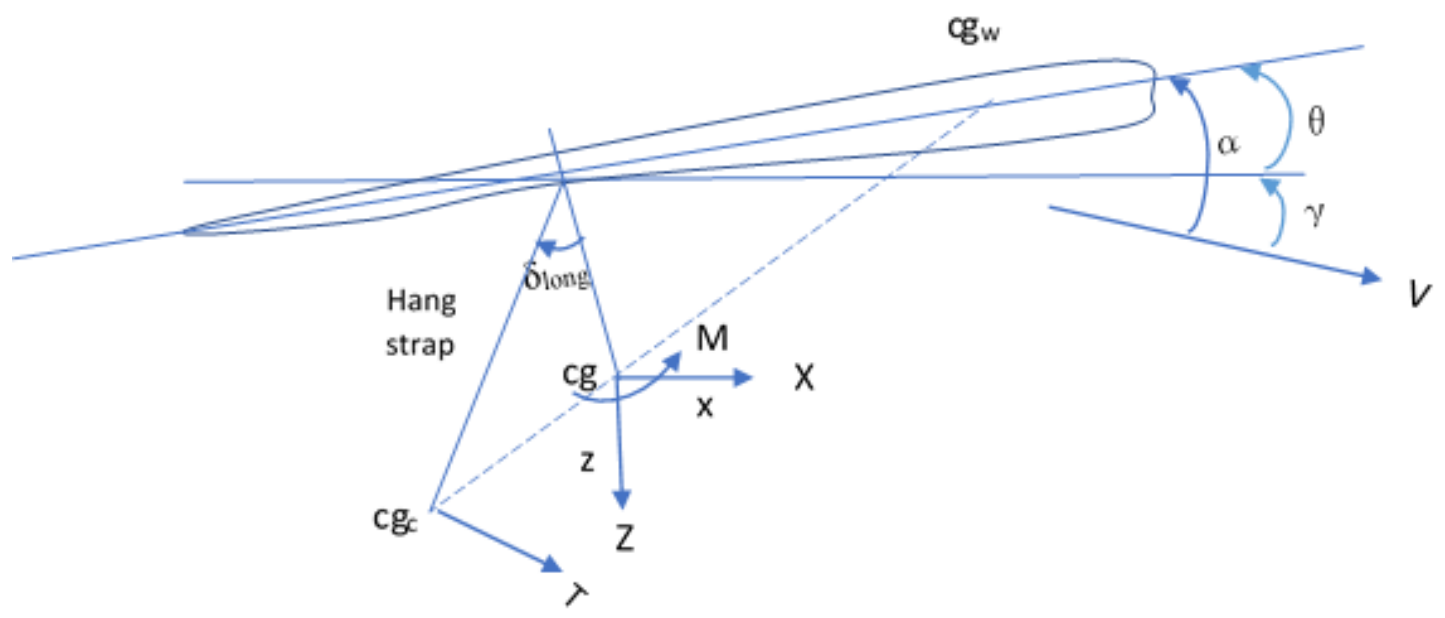

Figure 4.1: Longitudinal geometry

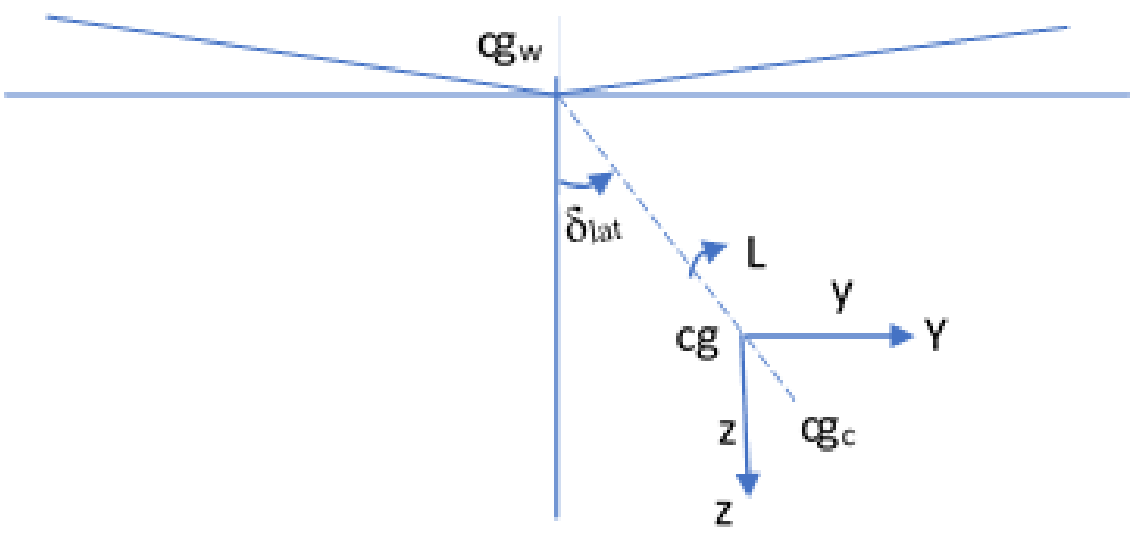

Figure 4.2: Lateral geometry 
Calculation of the behavior of the system is based on all the forces exerted on different sections of the vehicle. The first step is to apply the inertia law for different frames of reference to determine a steady control phase. The equilibrium equations derived from [4] and modified for an ultralight trike are as follows: Linear and angular momentum rate changes are equal to the total external force and moment around the mass center of the ultralight trike, and can be defined as follows:

$$
\begin{aligned}
& m(\dot{u}+q w)=X_{a}+X_{g} \\
& m(\dot{v}-p w+r u)=Y_{a}+Y_{g} \\
& m(\dot{w}+q u)=Z_{a}+Z_{g} \\
& I_{x} \dot{p}-I_{x z} \dot{r}=L_{a}+L_{g} \\
& I_{y} \dot{q}=M_{a}+M_{g} \\
& I_{z} \dot{r}-I_{x z} \dot{p}=N_{a}+N_{g}
\end{aligned}
$$

where $\mathrm{m}$ is the UAV mass, $\left(I_{x}, I_{y}, I_{z}\right)$ are moments of inertia about the $\mathrm{x}, \mathrm{y}, \mathrm{z}$ axis, $(\mathrm{L}, \mathrm{M}, \mathrm{N})$ are moments and $(\mathrm{u}, \mathrm{v}, \mathrm{w})$ linear velocity and $(\mathrm{p}, \mathrm{q}, \mathrm{r})$ are angular velocity.

The force and moment components can be further divided into two components gravitational and aerodynamic.

\subsubsection{The Forces and Moments of The Gravity}

$$
\begin{aligned}
& X_{g}=-m g(\sin \theta+\theta \cos \theta) \\
& Y_{g}=m g(\psi \sin \theta+\phi \cos \theta) \\
& Z_{g}=-m g(\theta \sin \theta-\cos \theta) \\
& L_{g}=M_{g}=N_{g}=0
\end{aligned}
$$


$\theta, \phi$, and $\psi$ are aircraft pitch, roll, and yaw angles, respectively.

\subsubsection{The Aerodynamic Forces and Moments}

The components of aerodynamic force and moments of a hang glider at the center of gravity (cg) consist of steady trim and perturbation contributions as follows [72]

$$
\begin{aligned}
X_{a}= & \left(X_{w}+X_{c}\right)_{e}+\left(X_{w}+X_{c}\right) \\
Y_{a}= & \left(Y_{w}+Y_{c}\right)_{e}+\left(Y_{w}+Y_{c}\right) \\
Z_{a}= & \left(Z_{w}+Z_{c}\right)_{e}+\left(Z_{w}+Z_{c}\right) \\
L_{a}= & \left(L_{w}+y_{w} Z_{w}-z_{w} Y_{w}\right)_{e}+\left(L_{c}+y_{c} Z_{c}-z_{c} Y_{c}\right)_{e} \\
& +\left(L_{w}+y_{w} Z_{w}-z_{w} Y_{w}\right)+\left(L_{c}+y_{c} Z_{c}-z_{c} Y_{c}\right) \\
M_{a}= & \left(M_{w}-x_{w} Z_{w}+z_{w} X_{w}\right)_{e}+\left(M_{c}-x_{c} Z_{c}+z_{c} X_{c}\right)_{e} \\
& +\left(M_{w}-x_{w} Z_{w}+z_{w} X_{w}\right)+\left(M_{c}-x_{c} Z_{c}+z_{c} X_{c}\right) \\
N_{a}= & \left(N_{w}+x_{w} Y_{w}-y_{w} X_{w}\right)_{e}+\left(N_{c}+x_{c} Y_{c}-y_{c} X_{c}\right)_{e} \\
& +\left(N_{w}+x_{w} Y_{w}-y_{w} X_{w}\right)+\left(N_{c}+x_{c} Y_{c}-y_{c} X_{c}\right)
\end{aligned}
$$

the subscripts w,c,and e refer to wing, trike, and steady equilibrium respectively. The flight can be considered as a steady trimmed symmetric equilibrium, therefore:

$$
\begin{aligned}
& \left(Y_{w}=L_{w}=N_{w}\right)_{e}=0 \\
& \left(Y_{c}=L_{c}=N_{c}\right)_{e}=0
\end{aligned}
$$

Equation (4.3) is composed of two types of forces and moments that can be included: steady trim and perturbation contributions. 


\subsubsection{Steady equilibrium}

By substituting Equation (4.4) in Equation (4.3), the equilibrium forces and moments can be determined as:

$$
\begin{aligned}
& \left(X_{w}+X_{c}\right)_{e}=L_{w} \sin \alpha-\left(D_{w}+D_{c}\right) \cos \alpha \\
& \left(Y_{w}+Y_{c}\right)_{e}=0 \\
& \left(Z_{w}+Z_{c}\right)_{e}=-L_{w} \cos \alpha-\left(D_{w}+D_{c}\right) \sin \alpha \\
& \left(y_{w} Z_{w}+z_{c} Z_{c}\right)_{e}=-y_{w}\left(L_{w} \cos \alpha+D_{w} \sin \alpha\right)-y_{c} D_{c} \sin \alpha \\
& \left(M_{w}-x_{w} Z_{w}+z_{w} X_{w}\right)_{e}+\left(M_{c}-x_{c} Z_{c}+z_{c} X_{c}\right)_{e}=M_{w} \\
& \quad+\left(x_{w} D_{w}+z_{w} L_{w}+x_{c} D_{c}\right) \sin \alpha+\left(x_{w} L_{w}-z_{w} D_{w}+z_{c} D_{c}\right) \cos \alpha \\
& \left(-y_{w} X_{w}+y_{c} X_{c}\right)_{e}=-y_{w}\left(L_{w} \sin \alpha-D_{w} \cos \alpha\right)+y_{c} D_{c} \cos \alpha
\end{aligned}
$$

\subsubsection{Perturbation forces and moments}

Small perturbations of the trim will generate unsteady aerodynamic forces and mo-

ments, which can be regarded as stability derivatives [4], [72]. Thus, perturbations of the wing are as follows:

$$
\begin{aligned}
& X_{w}=\dot{X}_{u} u_{w}+\dot{X}_{w} w_{w}+\dot{X}_{q} q_{w} \\
& Y_{w}=\dot{Y}_{v} v_{w}+\dot{Y}_{p} p_{w}+\dot{Y}_{r} r_{w} \\
& Z_{w}=\dot{Z}_{u} u_{w}+\dot{Z}_{w} w_{w}+\dot{Z}_{q} q_{w} \\
& L_{w}=\dot{L}_{v} v_{w}+\dot{L}_{p} p_{w}+\dot{L}_{r} r_{w} \\
& M_{w}=\dot{M}_{u} u_{w}+\dot{M}_{w} w_{w}+\dot{M}_{q} q_{w} \\
& N_{w}=\dot{N}_{v} v_{w}+\dot{N}_{p} p_{w}+\dot{N}_{r} r_{w}
\end{aligned}
$$

The perturbation velocities described in (4.6) with respect to the wing's reference 
system are orthogonal at its center of gravity.

Accordingly, (4.6) can be expressed with respect to the trick (cg) as follows:

$$
\begin{aligned}
& u_{w}=u+q z_{w} \\
& v_{w}=v-p z_{w}+r x_{w} \\
& w_{w}=w-q x_{w} \\
& p_{w}=p \\
& q_{w}=q \\
& r_{w}=r
\end{aligned}
$$

Substituting Equation(4.7) in Equation (4.6), will result in

$$
\begin{aligned}
& X_{w}=\dot{X}_{u} u+\dot{X}_{w} w+\left(z_{w} \dot{X}_{u}-x_{w} \dot{X}_{w}\right) q \\
& Y_{w}=\dot{Y}_{v} v-z_{w} \dot{Y}_{v} p+x_{w} \dot{Y}_{v} r \\
& Z_{w}=\dot{Z}_{u} u+\dot{Z}_{w} w+\left(\dot{Z}_{q}+z_{w} \dot{Z}_{u}-x_{w} \dot{Z}_{w}\right) q \\
& L_{w}=\dot{L}_{v} v+\left(\dot{L}_{p}-z_{w} \dot{L}_{v}\right) p+\left(\dot{L}_{r}+x_{w} \dot{L}_{v}\right) r \\
& M_{w}=\dot{M}_{u} u+\dot{M}_{w} w+\left(\dot{M}_{q}+z_{w} \dot{M}_{u}-x_{w} \dot{M}_{w}\right) q \\
& N_{w}=\dot{N}_{v} v+\left(\dot{N}_{p}-z_{w} \dot{N}_{v}\right) p+\left(\dot{N}_{r}+x_{w} \dot{N}_{v}\right) r
\end{aligned}
$$

As with the derivatives for the wing in (4.8), the presence of the trike will affect the motion equation equilibrium. The derivatives are expressed as follows: 


$$
\begin{aligned}
& \dot{X}_{q}=\dot{Z}_{q}=\dot{Y}_{p}=\dot{Y}_{r}=0 \\
& \dot{M}_{u}=\dot{M}_{w}=\dot{L}_{v}=\dot{L}_{r}=\dot{L}_{p}=\dot{N}_{v}=\dot{L}_{p}=0
\end{aligned}
$$

so,

$$
\begin{aligned}
& X_{c}=\dot{X}_{u} u+\dot{X}_{w} w+\left(z_{c} \dot{X}_{u}-x_{c} \dot{X}_{w}\right) q \\
& Y_{c}=\dot{Y}_{v} v-z_{c} \dot{Y}_{v} p+x_{c} \dot{Y}_{v} r \\
& Z_{c}=\dot{Z}_{u} u+\dot{Z}_{w} w+\left(z_{c} \dot{Z}_{u}-x_{c} \dot{Z}_{w}\right) q \\
& L_{c}=0 \\
& M_{w}=\dot{M}_{q} q \\
& N_{w}=\dot{N}_{r} r
\end{aligned}
$$

Mathematically, it is more effective to express the equations in terms of longitudinal and lateral control angles $(\zeta, \xi)[4]$.

\subsubsection{Longitudinal Control}

The Longitudinal geometry of the wing is shown in Fig. 4.3.

$$
\begin{aligned}
& x_{w}=\frac{m_{c}}{m}\left(l_{1}+l_{2} \sin \delta_{\text {long }}\right) \\
& x_{c}=-\frac{m_{w}}{m}\left(l_{1}+l_{2} \sin \delta_{\text {long }}\right) \\
& z_{w}=-\frac{m_{c}}{m} l_{2} \cos \delta_{\text {long }} \\
& z_{c}=\frac{m_{w}}{m} l_{2} \cos \delta_{\text {long }}
\end{aligned}
$$




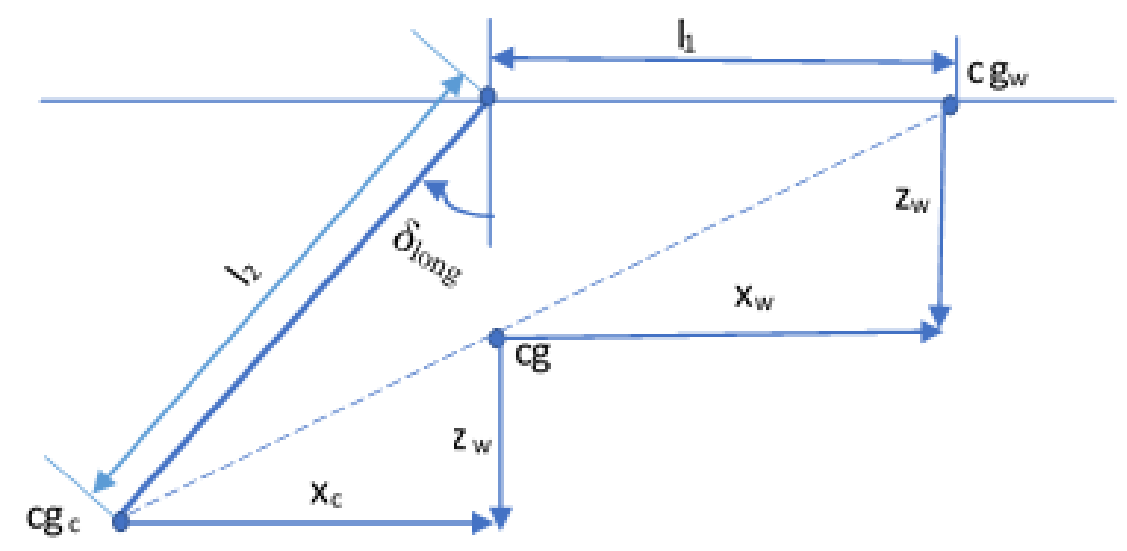

Figure 4.3: Longitudinal geometry

\subsubsection{Lateral Control}

The lateral geometry of wing is shown in Fig. 4.4.

$$
\begin{aligned}
& y_{w}=-\frac{m_{c}}{m} l_{2} \cos \delta_{\text {long }} \sin \delta_{\text {lat }} \\
& y_{c}=\frac{m_{w}}{m} l_{2} \cos \delta_{\text {long }} \sin \delta_{\text {lat }} \\
& z_{w}=-\frac{m_{c}}{m} l_{2} \cos \delta_{\text {long }} \cos \delta_{\text {lat }} \\
& z_{c}=\frac{m_{w}}{m} l_{2} \cos \delta_{\text {long }} \cos \delta_{\text {lat }}
\end{aligned}
$$

If the control angles of the wing can be divided into a steady equilibrium term and perturbation, then it may be expressed as

$$
\begin{gathered}
\delta_{\text {long }}=\left(\delta_{\text {long }}\right)_{e}+\delta_{\text {long }} \\
\delta_{\text {lat }}=\left(\delta_{\text {lat }}\right)_{e}+\delta_{\text {lat }}
\end{gathered}
$$

If the flight condition is a symmetric trim (i.e. $\left(\delta_{l a t}\right)_{e}=y_{w}=y_{p}=0$ ), position of the wing and the tricycle can be expressed as 


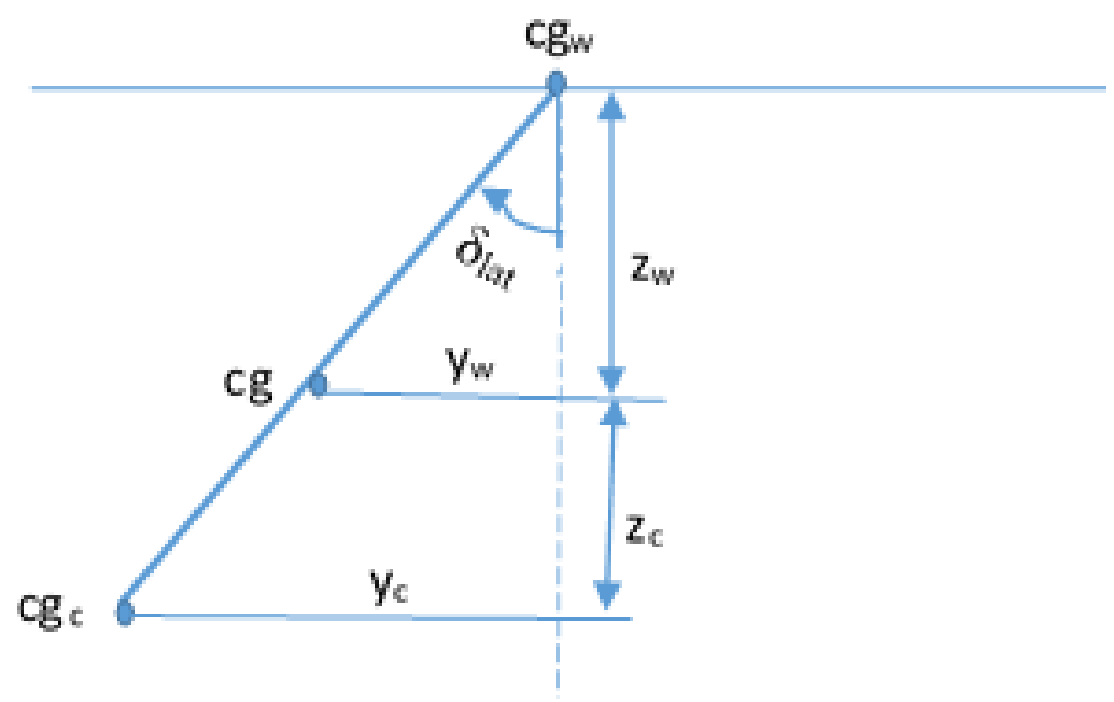

Figure 4.4: Lateral geometry

$$
\begin{aligned}
& x_{w e}+x_{w}=\frac{m_{c}}{m}\left(l_{1}+l_{2} \sin \left(\delta_{\text {long }}\right)_{e}+\left(l_{2} \cos \left(\delta_{\text {long }}\right)_{e}\right) \delta_{\text {long }}\right) \\
& x_{c e}+x_{c}=-\frac{m_{w}}{m}\left(l_{1}+l_{2} \sin \left(\delta_{\text {long }}\right)_{e}-\left(l_{2} \cos \left(\delta_{\text {long }}\right)_{e}\right) \delta_{\text {long }}\right) \\
& y_{w e}+y_{w}=-\frac{m_{c}}{m}\left(l_{2} \cos \left(\delta_{\text {long }}\right)_{e}\right) \delta_{\text {lat }} \\
& y_{c e}+y_{c}=\frac{m_{w}}{m}\left(l_{2} \cos \left(\delta_{\text {long }}\right)_{e}\right) \delta_{\text {lat }} \\
& z_{w e}+z_{w}=\frac{m_{c}}{m}\left(-l_{2} \cos \left(\delta_{\text {long }}\right)_{e}+\left(l_{2} \sin \left(\delta_{\text {long }}\right)_{e}\right) \delta_{\text {long }}\right) \\
& z_{c e}+z_{c}=-\frac{m_{w}}{m}\left(l_{2} \cos \left(\delta_{\text {long }}\right)_{e}-\left(l_{2} \sin \left(\delta_{\text {long }}\right)_{e}\right) \delta_{\text {long }}\right)
\end{aligned}
$$

\subsubsection{Total Aerodynamics With Thrust Force}

The thrust force normally reacts to the trike center of gravity $c g_{c}$ as illustrated in fig.

4.1. The total of the aerodynamic forces can be written as By substituting (4.10), (4.11), and (4.12) into (4.5), (4.8), and (4.9), and using Equation (4.3), we get: 


$$
\begin{aligned}
X_{a}= & L_{w} \sin \alpha-\left(D_{w}+D_{c}\right) \cos \alpha+\dot{X}_{u} u+\dot{X}_{w} w+\dot{X}_{q} q+\dot{X}_{\delta t h} \delta_{t h} \\
Y_{a}= & \dot{Y}_{v} v+\dot{Y}_{p} p+\dot{Y}_{r} r \\
Z_{a}= & -L_{w} \cos \alpha-\left(D_{w}+D_{c}\right) \sin \alpha+\dot{Z}_{u} u+\dot{Z}_{w} w+\dot{Z}_{q} q+\dot{Z}_{\delta t h} \delta_{t h} \\
L_{a}= & \dot{L}_{v} v+\dot{L}_{p} p+\dot{L}_{r} r+\dot{L}_{\xi} \xi+\dot{L}_{\delta t h} \delta_{t h} \\
M_{a}= & M_{w}+\left(x_{w} D_{w}+z_{w} L_{w}+x_{c} D_{c}\right) \sin \alpha+\left(x_{w} L_{w}-z_{w} D_{w}+z_{c} D_{c}\right) \cos \alpha \\
& +\dot{M}_{u} u+\dot{M}_{w} w+\dot{M}_{q} q+\dot{M}_{\delta} \delta+\dot{M}_{\delta t h} \delta t h \\
N_{w}= & \dot{N}_{v} v+\dot{N}_{p} p+\dot{N}_{r} r+\dot{N}_{\xi} \xi+\dot{N}_{\delta t h} \delta_{t h}
\end{aligned}
$$

where,

$$
\begin{aligned}
& \dot{L}_{\xi}=\frac{l_{2}}{m}\left(m_{w} Z_{c}-m_{c} Z w\right) \cos \delta \\
& \dot{M}_{\delta}=\xi=\frac{l_{2}}{m}\left(m_{w} Z_{c}-m_{c} Z w\right) \cos \delta+\xi+\left(m_{w} X_{c}-m_{c} X_{w}\right) \sin \delta \\
& \dot{N}_{\xi}=\frac{l_{2}}{m}\left(-m_{w} X_{c}+m_{c} X_{w}\right) \cos \delta \\
& \dot{X}_{\delta t h}=X_{\delta t h} \\
& \dot{Z}_{\delta t h}=Z_{\delta t h} \\
& \dot{M}_{\delta t h}=-\left(z_{p} X_{\delta t h}-x_{p} Z_{\delta t h}\right)
\end{aligned}
$$

\subsection{Equation of Motion Equilibrium}

By substituting (4.2) and (4.13) into (4.1), the equation of motion for the wing and the trike can be written in the form of a matrix, as follows:

1. For the longitudinal motion 


$$
\left[\begin{array}{cccc}
m & 0 & 0 & 0 \\
0 & m & 0 & 0 \\
0 & 0 & I_{y} & 0 \\
0 & 0 & 0 & 1
\end{array}\right]\left[\begin{array}{c}
\dot{u} \\
\dot{w} \\
\dot{q} \\
\dot{\theta}
\end{array}\right]=A_{\text {long }}\left[\begin{array}{l}
u \\
w \\
q \\
\theta
\end{array}\right]+B_{\text {long }}\left[\begin{array}{l}
\delta_{\text {long }} \\
\delta_{t h}
\end{array}\right]
$$

where,

$$
A_{\text {long }}=\left[\begin{array}{cccc}
\dot{X}_{u} & \dot{X}_{w} & \left(\dot{X}_{q}-m w_{e}\right) & -m g \cos \theta_{e} \\
\dot{Z}_{u} & \dot{Z}_{w} & \left(\dot{Z}_{q}+m u_{e}\right) & -m g \sin \theta_{e} \\
\dot{M}_{u} & \dot{M}_{w} & \dot{M}_{q} & 0 \\
0 & 0 & 1 & 0
\end{array}\right]
$$

and

$$
B_{l o n g}=\left[\begin{array}{cc}
0 & \dot{X}_{\delta t h} \\
0 & \dot{Z}_{\delta t h} \\
\dot{M}_{\delta} & \dot{M}_{\delta t h} \\
0 & 0
\end{array}\right]
$$

2. For lateral motion are 


$$
\left[\begin{array}{ccccc}
m & 0 & 0 & 0 & 0 \\
0 & I_{x} & -I_{x z} & 0 & 0 \\
0 & -I_{x z} & I_{z} & 0 & 0 \\
0 & 0 & 0 & 1 & 0 \\
0 & 0 & 0 & 0 & 1
\end{array}\right]\left[\begin{array}{c}
\dot{v} \\
\dot{p} \\
\dot{r} \\
\dot{\phi} \\
\dot{\psi}
\end{array}\right]=A_{\text {lat }}\left[\begin{array}{c}
v \\
p \\
\phi \\
\phi \\
\dot{L}_{\zeta} \\
\dot{N}_{\zeta} \\
0 \\
0
\end{array}\right] \delta_{\text {lat }}
$$

where,

$A_{\text {lat }}=\left[\begin{array}{ccccc}\dot{Y}_{v} & \left(\dot{Y}_{p}+m w_{e}\right) & \left(\dot{Y}_{r}-m u_{e}\right) & m g \cos \theta_{e} & m g \sin \theta_{e} \\ \dot{L}_{v} & \dot{L}_{p} & \dot{L}_{r} & 0 & 0 \\ \dot{N}_{v} & \dot{N}_{p} & \dot{N}_{r} & 0 & 0 \\ 0 & 1 & 0 & 0 & 0 \\ 0 & 0 & 1 & 0 & 0\end{array}\right]$

The coefficients for these equations and notions are shown in Appendix A. 


\section{Chapter 5}

\section{Control System Design}

\section{$5.1 \quad$ Introduction}

One challenge when designing the UAV flight control system is development of a robust nonlinear control technique that can nullify the effect of uncertainties and external disturbances, particularly the wind. The autopilot is the most important function of the in the autonomous system, as it guides the UAV to complete missions. It controls all flight phases of the aircraft: take-off, climbing, level flight, descent, landing, and loitering. This chapter presents the design of the complete UAV autopilot system, both longitudinal and lateral. It includes the longitudinal dynamics (forward speed, pitching and altitude) and the lateral dynamics (rolling and heading) [70], [71] and [42]. The autopilot design applied to the hang glider model depends on state space linear models (longitudinal and lateral) in [4], and the ANFIS controller design and comparison with conventional PID controller. The first role of the longitudinal motion controller is the design of most inner loops (pitch rate feedback) of the longitudinal system, then the pitch tracker design with ANFIS controller. The guidance and

control systems are related to the altitude hold controller of the ANFIS outer loop controller. The second task of the autopilot design is to develop the lateral motion 
controller, which has two stages: the inner loop (roll tracker) and the heading control which is related to guidance and control (outer loop).

\subsection{Longitudinal Control}

The longitudinal controller is responsible for controlling the velocity, pitch angle and altitude. This is done with two loops: the pitch tracker and the outer loop altitude (h) and the speed $\left(v_{a}\right)$ control. The controller produces a proper input value $\left(\delta_{\text {long }}\right)$ for a UAV based on an ANFIS controller, to track the reference values of altitude and airspeed. The control bar and the throttle are the inputs for the longitudinal motion controller. The control bar controls the inner loops of pitch and pitch rate and the outer loop height $(\mathrm{h})$, while the throttle $\left(\delta_{t}\right)$ is the outer loop control speed [71]. The response of the altitude loop is tested by climbing to a desired altitude (h) and executing straight and level flight. The velocity response loop functions in the same way.

\subsubsection{Inner Loop Pitch Attitude Tracker Design}

The longitudinal inner loop is the pitch attitude controller and the pitch damper (stability augmentation system (SAS)) [42]. The primary job of a pitch tracker or a pitch attitude hold is to maintain the value of the pitch attitude $(\theta)$ that matches the reference commanded pitch. Fig. 5.1 shows a block diagram of a pitch attitude holds control system. The design can be performed using the short-period period approximation for aircraft dynamics, and adding an integrator to obtain approximate pitch from the pitch rate. 


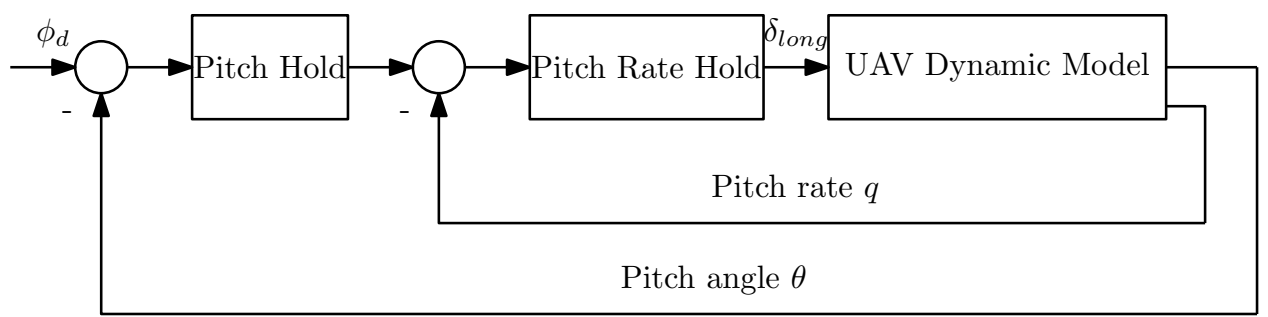

Figure 5.1: 1 Longitudinal Guidance and Control System for pitch hold

\subsubsection{Outer Loop Altitude Hold Controller}

The outer-loops were designed to meet the tracking command requirements for altitude and cruise speed, and the autopilot is used to track command requirements between the actual and the desired altitude. The flight altitude $\mathrm{h}(\mathrm{t})$ is not part of the state in the mathematical model, so it should modeled. In straight and level flight conditions, a change of altitude can be written as a function of the flight path angle and the velocity [72], so that

$$
\dot{h}=v_{a} \gamma=v_{a}(\theta-\alpha)
$$

The role of the longitudinal motion controller is to regulate straight, level flight, and climbing flight. Fig. 5.2 shows a block diagram of an altitude hold control system.

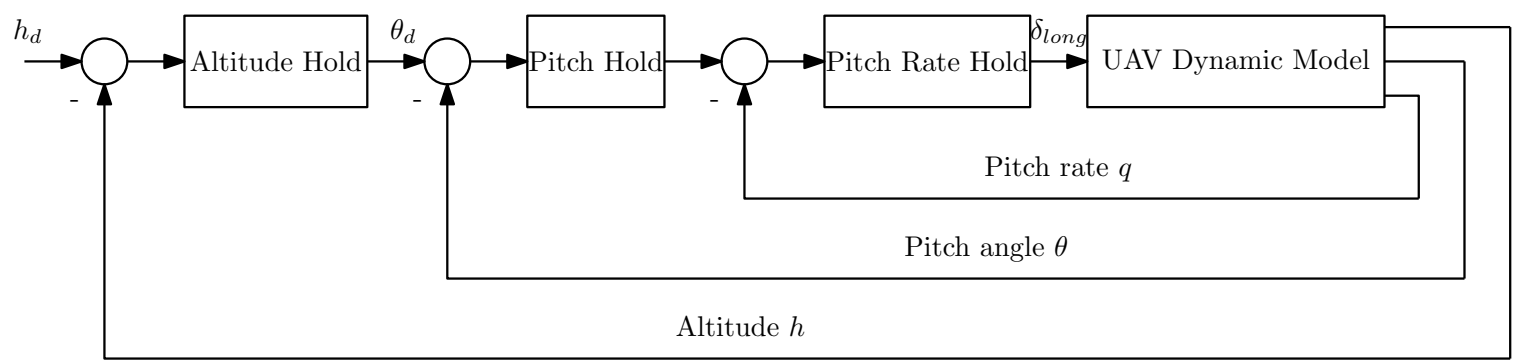

Figure 5.2: Longitudinal Guidance and Control System for altitude hold 


\subsubsection{Airspeed Hold Controller}

The airspeed hold control loop maintains constant speed along a flight path. It is designed based on the cruise mode requirement shown in Fig. 5.3, using the sensor measurement as input, and controlling the throttle. For speed changes, the throttle deflection is based on current speed error. The command goes directly to the aircraft model without modification by the inner loop, and ranges from of 0 and 1 .

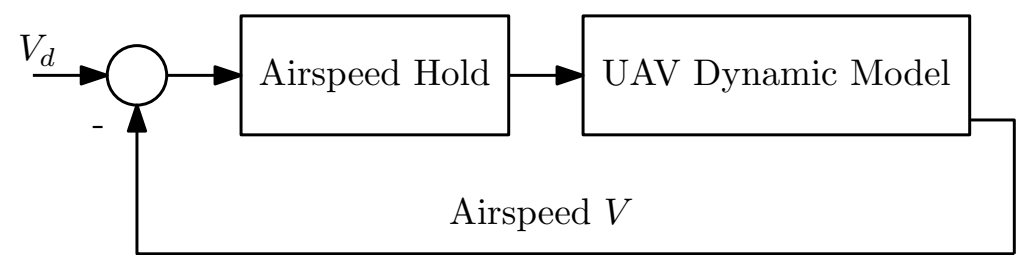

Figure 5.3: Longitudinal Guidance and Control System for speed hold

\subsection{Lateral Control}

This subsection focuses on the lateral control design of the direction controller, including both inner and outer loops. The outer loop uses heading signal (yaw angle) error to produce the reference command signal for the inner loop [73], and the inner loop uses command signal errors to create the deflection commands that are sent to the control components. The lateral controller produces a proper input value $\left(\delta_{l a t}\right)$.

\subsubsection{Inner Loops Roll Angle Hold Controller}

The roll attitude autopilot is designed to maintain the aircraft at wing level for long flights. The roll-angle-hold (bank angle hold) controller is controlled by the autopilot system as shown by the block diagram Fig. 5.4 [73]. Roll rate is designed to provide the inner loop rate damping for a good performance roll angle and to track the roll 
angle commands. The control bar controls the roll angle $\left(\delta_{l a t}\right)$.

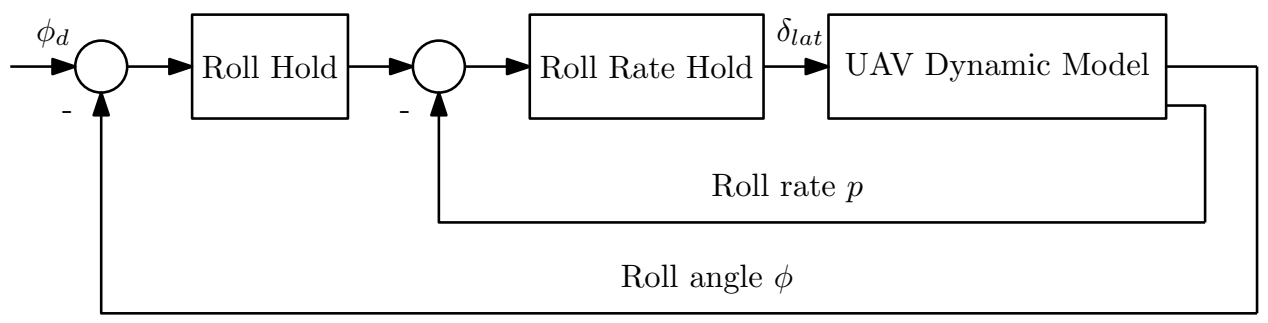

Figure 5.4: Lateral Guidance and Control System for roll hold

\subsubsection{Heading Hold Controller}

The heading-hold autopilot is largely designed to hold the aircraft on a specific heading, as shown in Fig. 5.5. The input command to the lateral autopilot is the desired heading, and the control bar command $\left(\delta_{\text {lat }}\right)$ is the output. The heading hold converts heading error to the roll attitude command, and the roll attitude hold transforms this error into a roll rate command. The roll rate hold turns roll rate errors into control bar commands $\left(\delta_{\text {lat }}\right)[73]$.

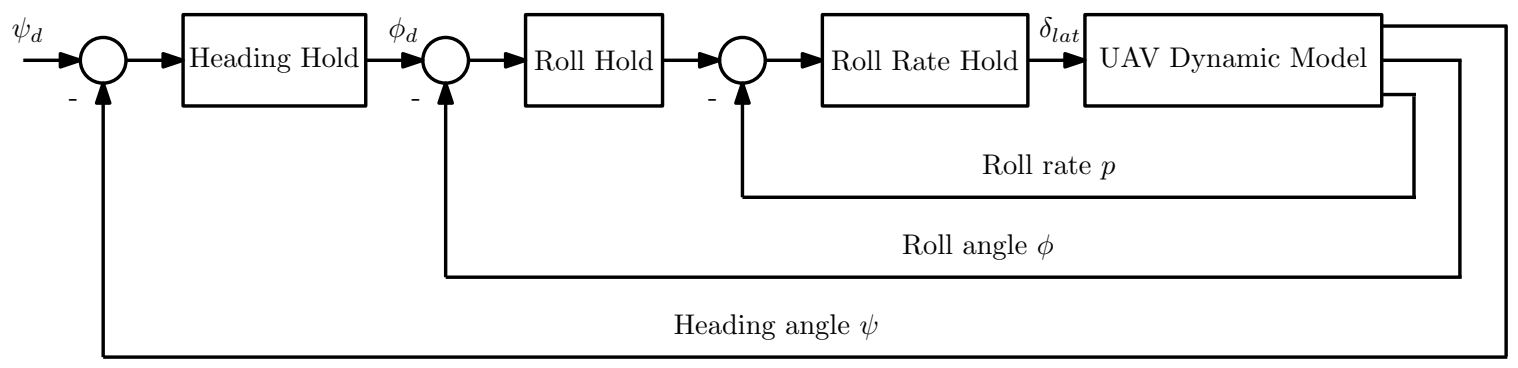

Figure 5.5: Lateral Guidance and Control System for heading hold 


\subsection{Controllability and Observability}

The concepts of controllability and observability were introduced by R. Kalman in 1960. They are two of the important properties of control systems.

The concepts of controllability and observability for a linear time-invariant dynamical system of flexible wing UAV can be related to suitable linear systems of algebraic equations. It is well known that a solvable system of linear algebraic equations has a solution if and only if the rank of the system matrix is full. Observability and controllability tests will be connected to the rank tests of certain matrices: the controllability and observability matrices. For the purpose of studying its observability, we consider the linear system:

$$
\begin{aligned}
& \dot{x}(t)=A x(t)+B u(t) \\
& y(t)=C x(t)+D u(t)
\end{aligned}
$$

Controllability determines whether it is possible to make system states evolve in time the way we want, using input system. To check whether particular system is controllable, matrix 5.3 could be used. If this matrix is full rank, then the system is said to be controllable [74].

$$
C_{n t r}=\left[\begin{array}{lllll}
B & A B & A^{2} B & \ldots & A^{n-1} B
\end{array}\right]
$$

Observability on the other hand, is to see if it is possible to determine all the states from output of the system and input signal. The linear continuous-time system and measurements in eq. 5.2 is observable if and only if the observability matrix has full rank. 


$$
O_{b s e}=\left[\begin{array}{c}
C \\
C A \\
C A^{2} \\
\vdots \\
C A^{n-1}
\end{array}\right]
$$

\subsection{PID Controller}

The proportional-integral-derivative (PID) control is one of the classic controller design techniques of UAV, based on a frequency domain analysis of system dynamics. A closed-loop controller uses feedback to control states or outputs of a dynamical system.

The feedback control is used to reduce the effect of uncertainty include disturbances and noise on the system [75]. There are many advantages of uses feedback controller then the open loop controllers:

1. Improve the response characteristics of the system.

2. Reject the effect of disturbance and noise.

3. Make the system stable.

Mathematically, a PID controller can be described as: [76]

$$
g=K_{p} e(t)+K_{i} \int_{0}^{t} e(t) d t+k_{d} \frac{d}{d t} e(t)
$$

where, $K_{p}, K_{i}$ and $K_{d}$ represent proportional, integral and derivative gains respectively. $\mathrm{T}$, is the control signal, which will be sent to the process. 
The PID controller commonly used in many industrial applications as easy to design and implement. The controller attempts to minimize the difference between desired and measurement signals as shown in Fig. 5.6. The controller is relatively easier to design and implement, compared to another type of controlle [77].

The Proportional gain reduces the rise time, steady-state error and noise at high frequencies. However, increasing the controller gain makes the system unstable.

The integral gain deals with a steady-state error by accelerating the movement of the process towards set-point. It can contribute to overshoot because it responds to accumulated error from the past that solved by adding the derivative term. The derivative gain slows down the rate change of the control signal and makes the overshoot smaller; it is no effect on the steady-state error. The combined controllerprocess stability is improved by the derivative term, but it makes the process unstable, and sensitive to noise in the error signal [78]. The procedure of the controller gains derived by using Ziegler-Nichols rule estimate interval change of PID parameters [79], [80]. The PID controller provides robust and reliable performance when the parameters are tuned well. The PID controller is a simply mathematical tuning system that lacks direct knowledge of the control process. It might cause a long settling time and constant overshoot until the error tends to zero

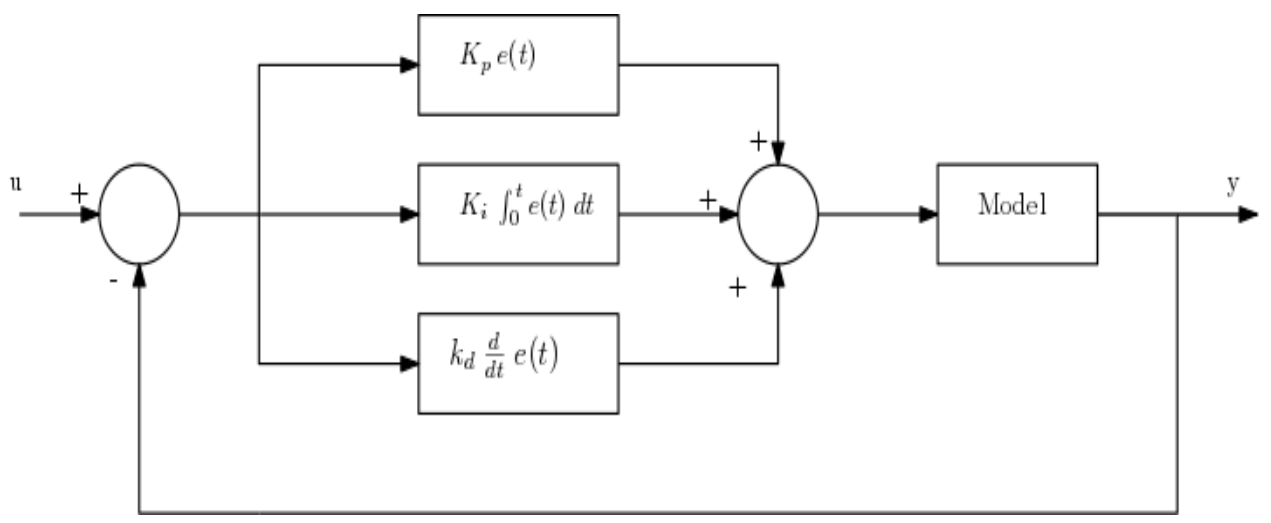

Figure 5.6: Block Diagram of PID Controller [76] 


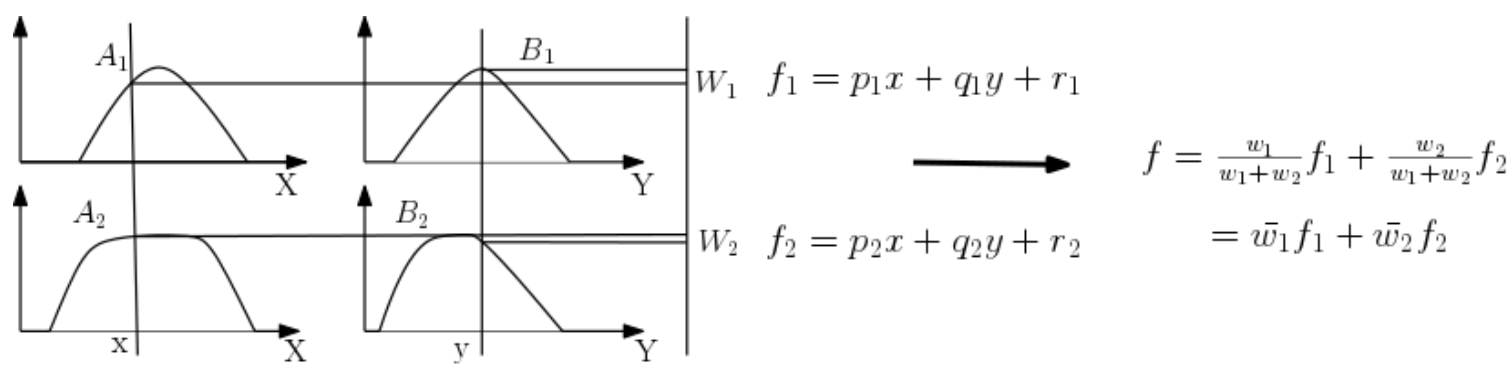

Figure 5.7: TSK fuzzy model [83]

\subsection{ANFIS Controller}

A neuro-fuzzy system was proposed by Jang [81]. An Adaptive Neuro-Fuzzy Inference Systems (ANFIS) [82] uses fuzzy logic and an artificial neural network. Fuzzy logic control deals with the uncertainty in complex problems, and it is an excellent tool for making decisions, based on their knowledge and database, but it can not learn. AN artificial neural network minimizes errors and optimizes the controller parameters but can not make decisions. Furthermore, the hybrid learning algorithm trains the membership functions, and fuzzy rules.

The ANFIS system and the Takagi-Sugeno fuzzy system are applied to automate fuzzy modeling by using measurement data. This consists of two input parameters, $\mathrm{x}$ and $\mathrm{y}$, and one output with two rules, as shown in Fig. 5.7 [83]. The total output is a weighted average of each rule's output:

Rule 1: if $\mathrm{x}$ is $A_{1}$ and $\mathrm{y}$ is $B_{1}$ then $f_{1}=p_{1} x+q_{1} y+r_{1}$

Rule 2: if $\mathrm{x}$ is $A_{2}$ and $\mathrm{y}$ is $B_{2}$ then $f_{2}=p_{2} x+q_{2} y+r_{2}$

where $\mathrm{x}$ and $\mathrm{y}$ are two inputs, $\mathrm{p}, \mathrm{q}$ and $\mathrm{r}$ are consequence parameters of the model resulting from the training procedure, and A and B are the fuzzy sets.

The ANFIS structure has five layers [81], as shown in Fig. 5.8.

Layer 1: The output for this layer $\left(L_{i}^{1}\right)$ is a membership function $\left(\mu_{A i}\right)$ of each input variable $(x$, and $y)$. 


$$
L_{A i}{ }^{1}=\mu_{A i}(x) \quad L_{B i}{ }^{1}=\mu_{B i}(y) \quad i=1, \ldots, n
$$

where $\mathrm{n}$ is the number of membership functions.

$$
\mu_{A i}(x)=\frac{1}{1+\left[\left(\frac{x-c_{i}}{a_{i}}\right)^{2}\right]^{b_{i}}}
$$

where $\left(a_{i}, b_{i}, c_{i}\right)$ is the parameter set that changes the shapes of the membership function. Parameters in this layer are referred to as the premise parameters.

Layer 2: This is the product of the two inputs.

$$
L_{i}^{2}=w_{i}=\mu_{A i}(x) \cdot \mu_{B i}(y) \quad i=1, \ldots, n
$$

Layer 3: Each node in this layer determines the normalized firing strength as follows:

$$
L_{i}^{3}=\bar{w}_{i}=\frac{w_{i}}{w_{1}+w_{2}} \quad i=1, \ldots, n
$$

Layer 4: The output is a summation of the previous outputs, such as:

$$
L_{i}^{4}=\bar{w}_{i}\left(p_{i} x+q_{i} y+r_{i}\right) \quad i=1, \ldots, n
$$

where $p_{i}, q_{i}$ and $r_{i}$ are the parameters from the training process.

Layer 5: This is the overall output and the final total of all the layers.

$$
L_{i}^{5}=\sum_{i} \bar{w}_{i}\left(p_{i} x+q_{i} y+r_{i}\right) \quad i=1, \ldots, n
$$




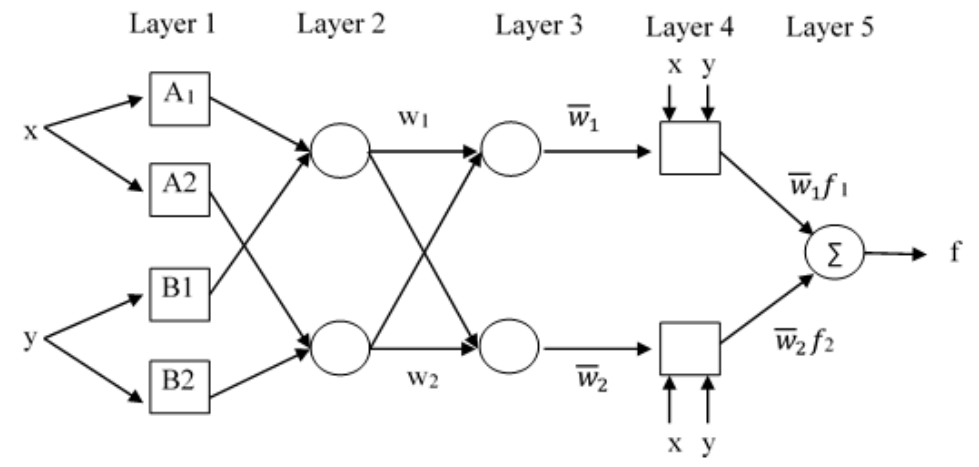

Figure 5.8: Adaptive neuro fuzzy interference system structure

\subsection{Hybrid Learning Algorithm}

The hybrid learning algorithm consist of two passes: forward pass and backward pass. During the forward pass of the hybrid learning algorithm, the parameters of the input membership functions are kept constant and since the output side functions are linear in parameters, the output parameters go forward until layer four and the consequent parameters are adjusted by using a least squares method [84].

When the premise parameters are fixed, the totally output can be expressed as a linear combination of the consequent parameters as:

$$
\begin{aligned}
f & =\frac{w_{1}}{w_{1}+w_{2}} f_{1}+\frac{w_{2}}{w_{1}+w_{2}} f_{2} \\
& =\bar{w}_{1} f_{1}+\bar{w}_{2} f_{2} \\
& =\left(\bar{w}_{1} x\right) p_{1}+\left(\bar{w}_{1} y\right) q_{1}+\left(\bar{w}_{1}\right) r_{1}+\left(\bar{w}_{2} x\right) p_{2}+\left(\bar{w}_{2} y\right) q_{2}+\left(\bar{w}_{2}\right) r_{2}
\end{aligned}
$$

During the backward pass, the output parameters are fixed, and the error propagate backward. The parameters of the input membership functions (premise parameters) are updated by gradient descent method. 


\section{Chapter 6}

\section{Sensor Fusion}

\subsection{Introduction}

This chapter presents the knowledge of sensor fusion to improve the attitude estimation of a flexible wing UAV equipped with DDVS and an accelerometer sensor. We first describe the attitude determination to estimate the Euler angles and the sensors. Then a new filter known as the Adaptive Unscented Kalman Filter (AUKF) is applied to integrate two DDVS/Accelerometer measurement sensors for attitude estimation.

\subsection{Attitude Determination}

Computation of UAV attitude involves the determination of UAV pitch, roll, and heading angles. Pitch angle and roll angle can be determined through inertial measurements such as accelerometers and rate gyros. The accelerometers measure the acceleration of the UAV. The rate gyros measure the rotational velocity of the UAV. Both sensors include sensor biases and zero mean Gaussian random noise. The rotation between the UAV body frame and the local Cartesian frame is determined from the attitude angles. 


$$
\left[\begin{array}{l}
\dot{\theta} \\
\dot{\phi} \\
\dot{\psi}
\end{array}\right]=\left[\begin{array}{ccc}
1 & \sin (\phi) \tan (\theta) & \cos (\phi) \tan (\theta) \\
0 & \cos (\phi) & -\sin (\phi) \\
0 & \sin (\phi) \sec (\theta) & \cos (\phi) \sec (\theta)
\end{array}\right]\left[\begin{array}{l}
p \\
q \\
r
\end{array}\right]
$$

where $\theta, \phi$, and $\psi$ are pitch, roll and heading angles, the $\mathrm{p}, \mathrm{q}$, and $\mathrm{r}$ are angular velocities.

The pitch and roll angles can be determined either by computing the gravitational acceleration components on the body axes, or by using the Euler quaternion method [85]. The gravitational acceleration components give long-term accuracy and accompanied by high noise contents. While the quaternion method gives low noise contents and fast response, but tends to drift with time due to gyro bias errors. A common method for computing the attitude angle is the Euler quaternion method [86], which uses the Euler four symmetrical parameters $\left(q_{0}, q_{1}, q_{2}, q_{3}\right)$ to define the UAV attitude. The relationship between the attitude angle and the four parameters are [87] [88]:

$$
\left[\begin{array}{l}
\phi \\
\theta \\
\psi
\end{array}\right]=\left[\begin{array}{c}
\operatorname{atan}\left(\frac{2\left(q_{0} q_{2}-q_{1} q_{3}\right.}{q_{0}^{2}+q_{1}^{2}-q_{2}^{2}-q_{3}^{2}}\right) \\
\operatorname{asin}\left(2 q_{0} q_{3}+2 q_{1} q_{2}\right) \\
\operatorname{atan}\left(\frac{2\left(q_{0} q_{1}-q_{2} q_{3}\right.}{q_{0}^{2}-q_{1}^{2}+q_{2}^{2}-q_{3}^{2}}\right)
\end{array}\right]
$$


The kinematics equation (6.1) in quaternion components is:

$$
\left[\begin{array}{c}
\dot{q}_{0} \\
\dot{q}_{1} \\
\dot{q}_{2} \\
\dot{q_{3}}
\end{array}\right]=\frac{1}{2}\left[\begin{array}{cccc}
0 & -p & -q & -r \\
p & 0 & r & -q \\
q & -r & 0 & p \\
r & q & -p & 0
\end{array}\right]\left[\begin{array}{l}
q_{0} \\
q_{1} \\
q_{2} \\
q_{3}
\end{array}\right]
$$

where the quaternion components driven from Euler angles are following:

$$
\begin{aligned}
& q_{0}=\cos \frac{\phi}{2} \cos \frac{\theta}{2} \cos \frac{\psi}{2}+\sin \frac{\phi}{2} \sin \frac{\theta}{2} \sin \frac{\psi}{2} \\
& q_{1}=\sin \frac{\phi}{2} \cos \frac{\theta}{2} \cos \frac{\psi}{2}-\cos \frac{\phi}{2} \sin \frac{\theta}{2} \sin \frac{\psi}{2} \\
& q_{2}=\cos \frac{\phi}{2} \sin \frac{\theta}{2} \cos \frac{\psi}{2}+\sin \frac{\phi}{2} \cos \frac{\theta}{2} \sin \frac{\psi}{2} \\
& q_{3}=\cos \frac{\phi}{2} \cos \frac{\theta}{2} \sin \frac{\psi}{2}+\sin \frac{\phi}{2} \sin \frac{\theta}{2} \cos \frac{\psi}{2}
\end{aligned}
$$

And the quaternion is normal i.e $\left(q_{0}^{2}+q_{1}^{2}+q_{2}^{2}+q_{3}^{2}=1\right)$

\subsection{Accelerometer}

Accelerometer sensors are used in a variety of automation applications. They measure linear acceleration during static or quasi-static conditions and calculate the angles relative to the horizontal plane; this information determines the pitch and roll angles of the rigid body [89]. The orientation of the flexible wing is measured by a 3-axis ADXL 330 accelerometer with full-scale range of $3 \mathrm{~g}$, as shown in Fig. 6.1, and based on 
MEMS technology from Analog Devices. The onboard sensors can only communicate if there is a microcontroller reading the sensor values and processing the data signals.

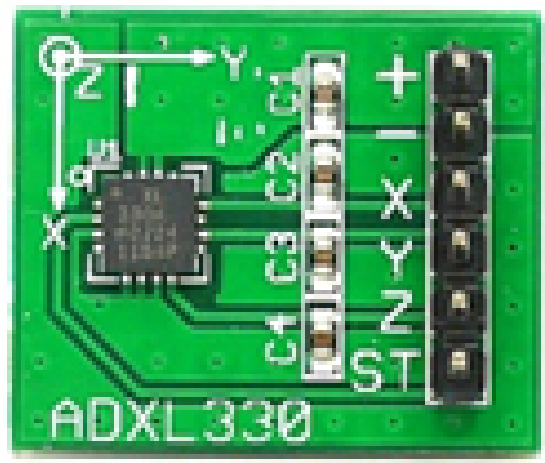

Figure 6.1: ADXL 330 accelerometer sensor

The Arduino Uno R3 in Fig. 6.2 can produce the proper signals without extra configuration. Accelerometers provide roll and pitch angles using gravity, and their performance regarding noise(noise density expressed in $\mathrm{g} / \mathrm{Hz}$ ) is supplied by the manufacturer, as presented in Table 6.1. The 3-axis ADXL 330 has good performance with respect to the noise density.

The roll and pitch angles are estimated by the following equations:

$$
\begin{gathered}
\theta=\operatorname{atan} \frac{a_{x}}{\sqrt{a_{y}^{2}+a_{z}^{2}}} \\
\phi=\operatorname{atan} \frac{-a_{y}}{a_{z}}
\end{gathered}
$$

where $\theta$ and $\phi$ are the pitch and the roll angles estimated from the accelerometer outputs, respectively, and $a_{x}, a_{y}$ and $a_{z}$ are the components of the acceleration measured by the accelerometer in the body coordinate frame. 
Table 6.1: Accelerometer noise density from manufacture

\begin{tabular}{|c|l|}
\hline & Noise Density $[\mu g / \sqrt{H z}]$ \\
\hline x-axis & 280 \\
\hline y-axis & 280 \\
\hline z-axis & 350 \\
\hline
\end{tabular}

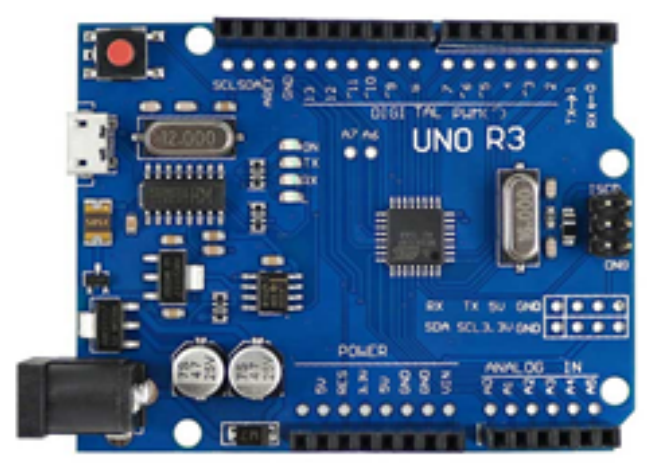

Figure 6.2: Arduino Uno R3 board

\subsection{Sensor Fusion}

This section examines the proposed sensor fusion. The methodology is used to calculate attitude estimation by integrating the reference data that is gathered from DDVS and measurement data from the accelerometer. The algorithm is designed to optimize and robust the DDVS for pitch and roll angles. The sensor fusion algorithm is based on the Adaptive Unscented Kalman Filter (AUKF), and is applied to estimate the state variables (attitude estimate). The input measurements to predicte state variables are inertial accelerations according to the accelerometer and DDVS results. The block diagram of the sensor fusion algorithm using AUKF is shown in Fig. 6.3.

Errors associated with the accelerometer are affected by the results, and diverge with time [91]. The DDVS measurements are used in the AUKF to estimate the errors and obtain accurate and corrected attitudes. 


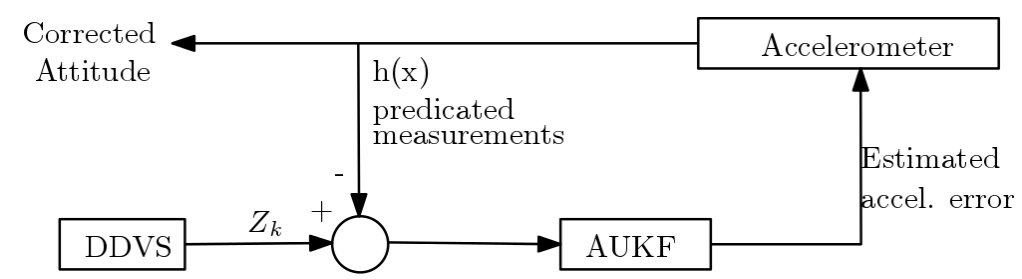

Figure 6.3: Block diagram of AUKF

\subsection{Adaptive Kalman Filter (AUKF)}

It is difficult to design a UAV autopilot controller without considering the effect of signal noise and disturbance on the measurement state values; AUKF is designed to remove these impediments and estimate the actual state values. Fig. 6.4, illustrated a system based on a robust ANFIS controller and the propose DDVS, as well as a new AUKF to control the longitudinal and lateral autopilot of a flexible wing UAV.

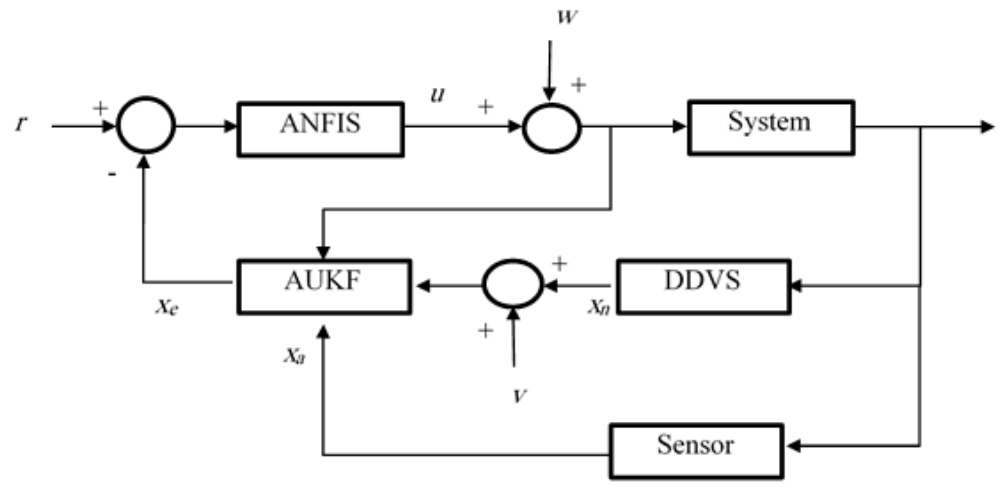

Figure 6.4: The feedback control system for the flexible wing UAV

This system ensures system stability and improves the performance of a system under the noise conditions. The AUKF uses the Fuzzy Logic Adaptive System (FLAS) to prevent the estimate results from divergence [91]. It evaluate the UKF performance and tunes the factors in the weighted covariance to improve UKF accuracy [92]. The state estimate of DDVS and the sensor are $x_{n}$ and $x_{a}$ respectively, and the estimation output is $x_{e}$, as shown in Fig. 6.4. Thus the state form equation is: 


$$
\begin{gathered}
x(k+1)=A(k) x(k)+B(k) u(k)+w(k) \\
z(k)=H(k) x(k)+v(k)
\end{gathered}
$$

where $\mathrm{A}(\mathrm{k})$ is the transition matrix, $\mathrm{x}(\mathrm{k})$ the state vector, $\mathrm{B}(\mathrm{k})$ the control matrix, $\mathrm{u}(\mathrm{k})$ the input vector, $\mathrm{w}(\mathrm{k})$ the process noise, $\mathrm{z}(\mathrm{k})$ the measurement vector, $\mathrm{H}(\mathrm{k})$ the measurement matrix and $\mathrm{v}(\mathrm{k})$ the measurement noise. No correlation between $\mathrm{w}(\mathrm{k})$ and $\mathrm{v}(\mathrm{k})$ is assumed [91]:

$$
\begin{gathered}
E\left[w(k) w(i)^{T}\right]= \begin{cases}Q & \text { if } i=k \\
0 & \text { if } i \neq k\end{cases} \\
E\left[v(k) v(i)^{T}\right]= \begin{cases}R & \text { if } i=k \\
0 & \text { if } i \neq k\end{cases} \\
E\left[w(k) v(i)^{T}\right]=0
\end{gathered}
$$

\subsubsection{Unscented Kalman Filter}

The UKF algorithm produces several sampling points (sigma points) around the mean, based on the covariance. These points are propagated through nonlinear transformation (Unscented transformation (UT)) to improve the accuracy of the results [90], [92]. The UT is an alternative method to the analytical linearization approach. As random variable $\mathrm{x}$ of $\mathrm{n}$-dimensions with mean $\widehat{x}$ and covariance $p_{x}$ can be increased using a nonlinear function.

The UKF algorithm is summarized as follows [90]: 
1. The sigma points $\mathrm{X}$ and weighted points $\mathrm{W}$ can be determined as follows:

$$
\begin{aligned}
& X=\left[\widehat{x} \quad \widehat{x} \pm\left(\sqrt{(n+\lambda) p_{x}}\right)_{i}\right] ; i=1,2, \ldots .2 n \\
& W_{0}^{(m)}=\frac{\lambda}{n+\lambda} \\
& W_{0}^{(c)}=W_{0}^{(m)}+\left(1-\alpha^{2}+\beta\right) \\
& W_{i}^{(c)}=W_{i}^{(m)}=\frac{1}{2(n+\lambda)} i=1,2, \ldots 2 n
\end{aligned}
$$

The scaling parameter is computed as $\lambda=\alpha^{2}(n+k)-n, \alpha$ computes the sigma points spread around $\widehat{x}$ and it is a small positive value (e.g. $0.0001 \leq \alpha \leq 1)$; $\mathrm{k}$ is scaling parameter (typically 0), and the optimal choice for Gaussians is $\beta=2$. The weight of the mean and covariance are represented by Equations $6.12, \quad 6.13, \quad 6.14$ and 6.15 [92].

2. Each sigma point is transformed through the nonlinear state prediction function, $f$.

$$
\left(\widehat{X}_{k, k-1}\right)_{i}=f\left((X)_{i}\right) i=0,1, \ldots 2 n
$$

3. Predicted mean value using weighted averages of the transformed sigma-points is calculated by:

$$
\left(\widehat{x}_{k, k-1}\right)_{i}=\sum_{i=0}^{2 n} W_{i}^{(m)}\left(\widehat{X}_{k, k-1}\right)_{i}
$$


4. Predicted covariance is calculated by:

$$
\begin{aligned}
P_{k, k-1}= & \sum_{i=0}^{2 n} W_{i}^{(c)}\left[\widehat{X}_{k, k-1}-\widehat{x}_{k, k-1}\right]\left[\widehat{X}_{k, k-1}-\widehat{x}_{k, k-1}\right]^{T} \\
& +Q
\end{aligned}
$$

5. Prediction points are calculated through observation model:

$$
\left(\widehat{Z}_{k, k-1}\right)_{i}=h\left(\widehat{X}_{k, k-1}\right)_{i}
$$

6. Predicted observation:

$$
\widehat{z}_{k, k-1}=\sum_{i=0}^{2 n} W_{i}^{(m)}\left(\widehat{Z}_{k, k-1}\right)_{i}
$$

7. Innovation covariance:

$$
\begin{aligned}
P_{v v}= & \sum_{i=0}^{2 n} W_{i}^{(c)}\left[\widehat{Z}_{k, k-1}-\widehat{z}_{k, k-1}\right]\left[\widehat{Z}_{k, k-1}-\widehat{z}_{k, k-1}\right]^{T} \\
& +R
\end{aligned}
$$

8. Cross covariance:

$$
P_{x z}=\sum_{i=0}^{2 n} W_{i}^{(c)}\left[\widehat{X}_{k, k-1}-\widehat{x}_{k, k-1}\right]\left[\widehat{Z}_{k, k-1}-\widehat{z}_{k, k-1}\right]^{T}
$$

9. Update:

$$
\begin{gathered}
K_{k}=P_{x z} P_{v v}^{-1} \\
\widehat{x}_{k}=\widehat{x}_{k, k-1}+K_{k}\left(z_{k}-\widehat{z}_{k, k-1}\right)
\end{gathered}
$$




$$
P_{k}=P_{k, k-1}-K_{k} P_{v v} K_{k}^{T}
$$

\subsubsection{Fuzzy Logic Adaptive System (FLAS)}

Fuzzy logic is used with the mathematical model to prevent UKF from divergence [91] [92]. In general, a fuzzy system consists of three components: fuzzification, fuzzy reasoning (fuzzy inference), and defuzzification. The fuzzification stage converts a crisp input value to a linguistic value, the fuzzy inference is responsible for fuzzy rules and fuzzy de-fuzzification converts the fuzzy output to a crisp value.

As the input of the fuzzy inference engine, the Degree of Divergence (DOD) has two parameters. The first is $\mu$ which is derived from the mean of residuals $v$ [93], [94] as follows:

$$
\mu=\frac{1}{n} \sum_{1-n}^{n} v_{i}
$$

The second DOD parameter $\xi$ is the covariance of residuals as follows:

$$
\xi=\frac{v_{k}^{T} v_{k}}{n}
$$

where $\mathrm{n}$ is the measurement number and, $v_{k}$ is an innovation covariance.

$$
v_{k}=z_{k}-\hat{z}_{k}
$$

By monitoring the DOD parameters with fuzzy logic, the softening factor $\alpha$ (the output of FLAS) is tuned to the fuzzy rules and estimation accuracy, as shown in Fig. 6.5. As there are no specific criteria to find the softening factor, it must be determined empirically.

The models of weighted noise covariance matrices for EKF are presented as obtained by Sasiadek [93]. The set of covariance matrices are as follows: 


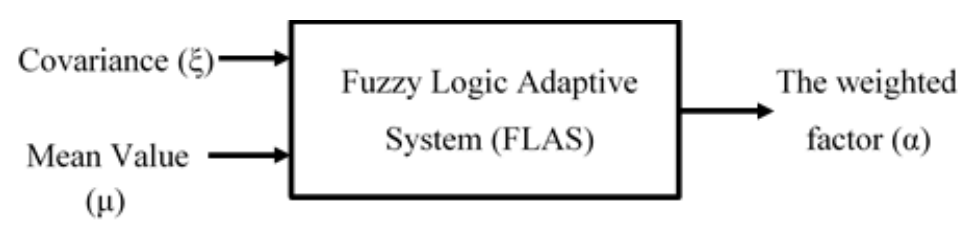

Figure 6.5: The input and output of FLAS

$$
\begin{aligned}
& R_{k}=R \alpha^{-2(k+1)} \\
& Q_{k}=Q \alpha^{-2(k+1)}
\end{aligned}
$$

where $\alpha \geq 1$ and matrices $\mathrm{Q}$ and $\mathrm{R}$ are constants. When $\mathrm{k}$ increases, the $\mathrm{R}$ and Q matrices decrease, and the most higher measurement gets the most weighting, Therefore, the weighted covariance is:

$$
P_{k, k-1}^{\alpha}=P_{k, k-1} \alpha^{2 k}
$$

Substituting Equation(6.31) into Equations(6.21 ,6.22, and 6.25) we obtain,

$$
\begin{aligned}
P_{k, k-1}= & \alpha^{2} \sum_{i=0}^{2 n} W_{i}^{(c)}\left[\widehat{X}_{k, k-1}-\widehat{x}_{k, k-1}\right]\left[\widehat{X}_{k, k-1}-\widehat{x}_{k, k-1}\right]^{T} \\
& +Q \\
P_{v v}= & \alpha^{2 k} \sum_{i=0}^{2 n} W_{i}^{(c)}\left[\widehat{Z}_{k, k-1}-\widehat{z}_{k, k-1}\right]\left[\widehat{Z}_{k, k-1}-\widehat{z}_{k, k-1}\right]^{T} \\
& +\alpha^{-2} R \\
P_{x z}= & \alpha^{2 k} \sum_{i=0}^{2 n} W_{i}^{(c)}\left[\widehat{X}_{k, k-1}-\widehat{x}_{k, k-1}\right]\left[\widehat{Z}_{k, k-1}-\widehat{z}_{k, k-1}\right]^{T}
\end{aligned}
$$




\section{Chapter 7}

\section{Experimental Setup and Results}

\subsection{Introduction}

This chapter discusses the identification, classification and control of flexible wing UAV by machine vision that has been implemented and tested. Initially, the distinguishing features of the wing are extracted and 3D points are computing for each point using machine vision as described in Chapter three. Fuzzy logic classify the shape of the wing and ANFIS and PID controllers are used to design the autopilot. All simulation results were verified and validated.

\subsection{Machine Vision}

The most important methods of image processing, feature extraction, pose estimation, and tracking algorithms applied in this work were tested and discussed. To verify the performance of all algorithms for pose estimation and determine the translation and rotation between the two cameras, we should calibrated the camera and then calculated the intrinsic matrix K. The camera (Microsoft LifeCam HD-3000 are shown in Table 7.1. 
Table 7.1: Specification of Microsoft LifeCam HD-3000

\begin{tabular}{|l|l|}
\hline \multicolumn{2}{|l|}{ Specifications of Camera } \\
\hline Product Name & Microsoft LifeCam HD-3000 \\
\hline & \\
\hline Webcam Length & 1.55 inches (39.3 millimeters) \\
\hline Webcam Width & 1.75 inches (44.5 millimeters) \\
\hline Webcam Depth/Height & 4.28 inches (109 millimeters) \\
\hline Webcam Weight & 3.17 ounces (89.9 grams) \\
\hline Interface & $\begin{array}{l}\text { High-speed USB compatible } \\
\text { with the USB } 2.0\end{array}$ \\
\hline Imaging Rate & Up to 30 frames per second \\
\hline Field of View & 68.5 diagonal field of view \\
\hline Fixed focus & from 0.3m to 1.5m \\
\hline True Color & $\begin{array}{l}\text { Automatic image adjustment } \\
\text { with manual override }\end{array}$ \\
\hline Imaging Features & $\begin{array}{l}16: 9 \text { widescreen } \\
24 \text {-bit } \\
\text { color depth }\end{array}$ \\
\hline
\end{tabular}




\subsubsection{Camera Calibration}

The first step of camera calibration is to find a relationship between the object that appears on images and where it is in the world coordinate system. This involves calculating intrinsic parameters such as focal length, principal point and lens distortion, and extrinsic parameters such as the 3D position and orientation of the camera with respect to the world coordinate system. The inputs for this are several images of a model chessboard plane with calibration points, using corners of the plane as calibration points. Fig. 7.1 and 7.2 show calibration images.

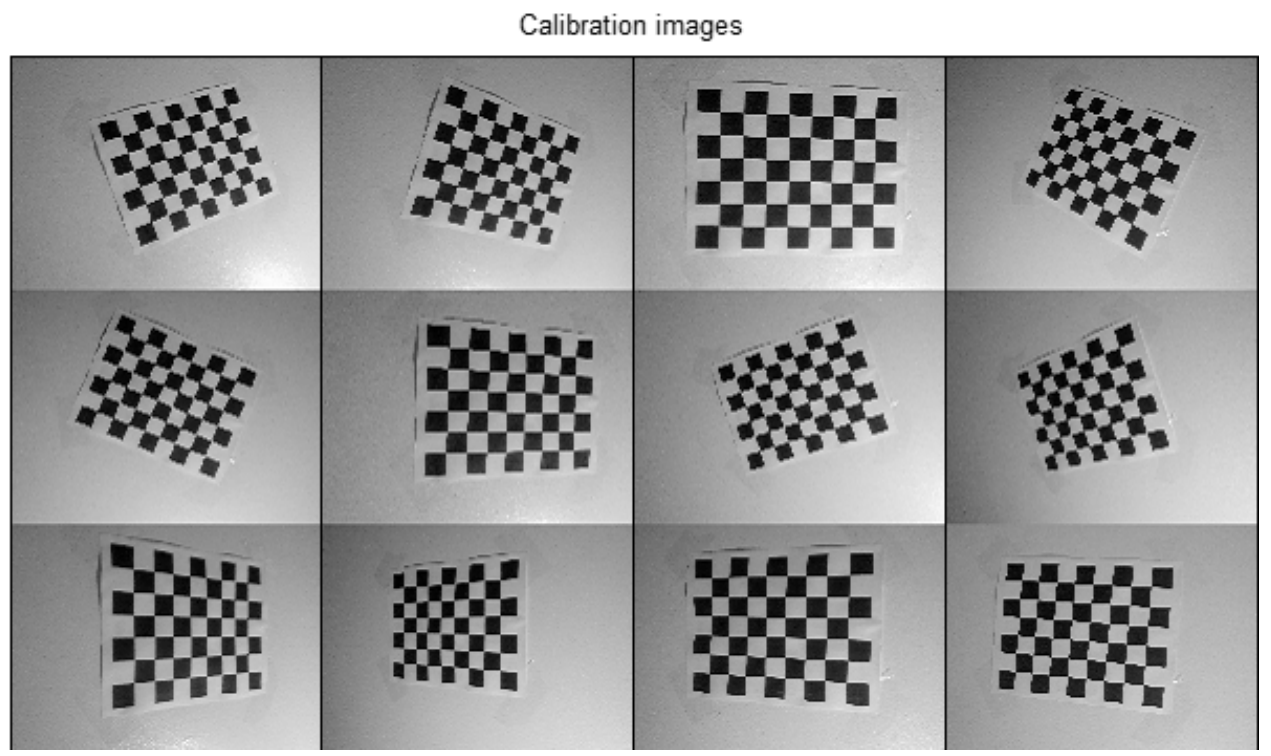

Figure 7.1: Camera calibration toolbox for MATLAB with a model chessboard Plane 


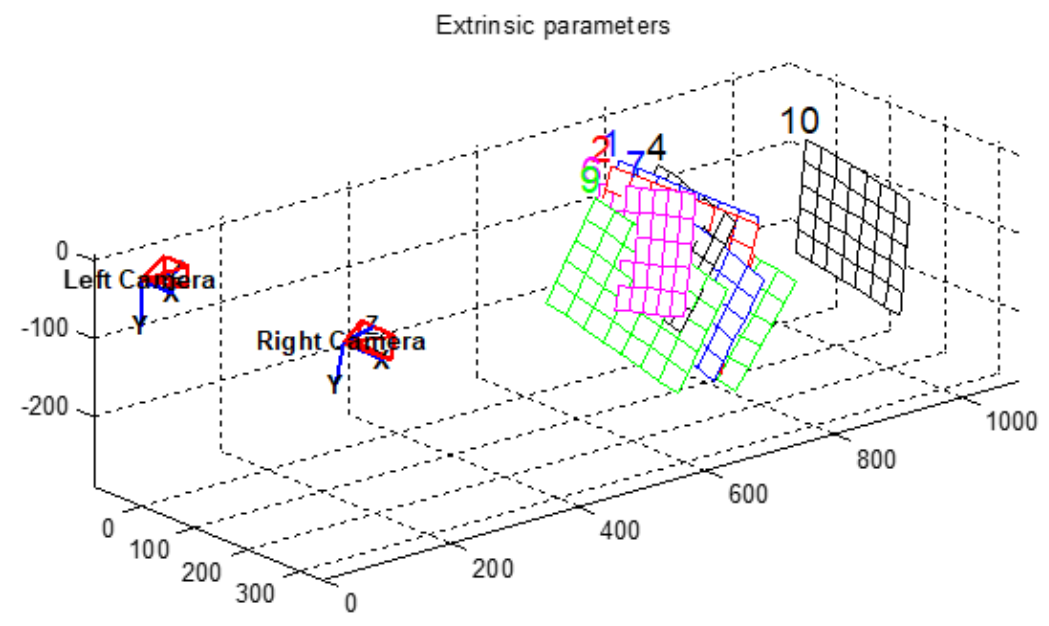

Figure 7.2: Camera extrinsic parameters

The $\mathrm{k}$ matrix for Microsoft LifeCam is:

$$
k=\left[\begin{array}{ccc}
2380.8 & 0 & 1277 \\
0 & 2371.6 & 935.4 \\
0 & 0 & 1
\end{array}\right]
$$

While the $\mathrm{k}$ matrix for ZED stereo camera is:

$$
k_{\text {left }}=\left[\begin{array}{ccc}
700.819 & 0 & 665.465 \\
0 & 700.819 & 371.953 \\
0 & 0 & 1
\end{array}\right]
$$




$$
k_{\text {right }}=\left[\begin{array}{ccc}
698.76 & 0 & 640.082 \\
0 & 698.76 & 375.129 \\
0 & 0 & 1
\end{array}\right]
$$

\subsubsection{The Error Functions}

We use the error in the second image to evaluate the algorithms after transferring points from the first image using the computed homography matrix. All the methods were programmed and tested under the same image noise conditions. A Euclidean image is the distance in the second image between the measured point $x_{i}^{*}$ and the point $\mathrm{H} x_{i}$. If $\mathrm{d}$ is the Euclidean distance between inhomogeneous points, the transfer error in the set of correspondences is

$$
E=\sum_{i} d\left(x_{i}^{*}-H x_{i}\right)^{2}
$$

The performance of three algorithms (DLT, RANSAC and Pseudo-inverse) in fourlevel Gaussian noise conditions was compared in Fig. 7.3. All performances degraded as the noise level increased, through the RANSAC algorithm had the better results than the others. To verify the decomposition homography algorithm performance, we added the same noise error to the second image and calculated the relative translation error as shown in Fig. 7.4. It is a clear that all errors increased proportionately with the level of noise added to the image feature points. 


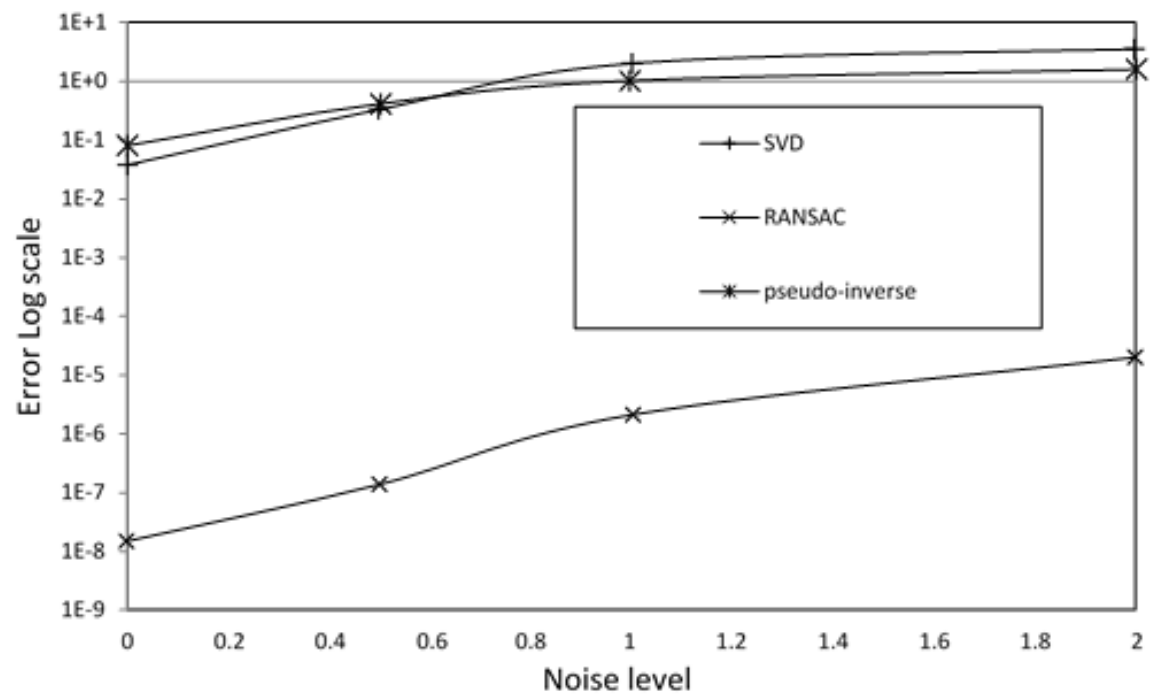

Figure 7.3: Plot of the log error against the noise levels

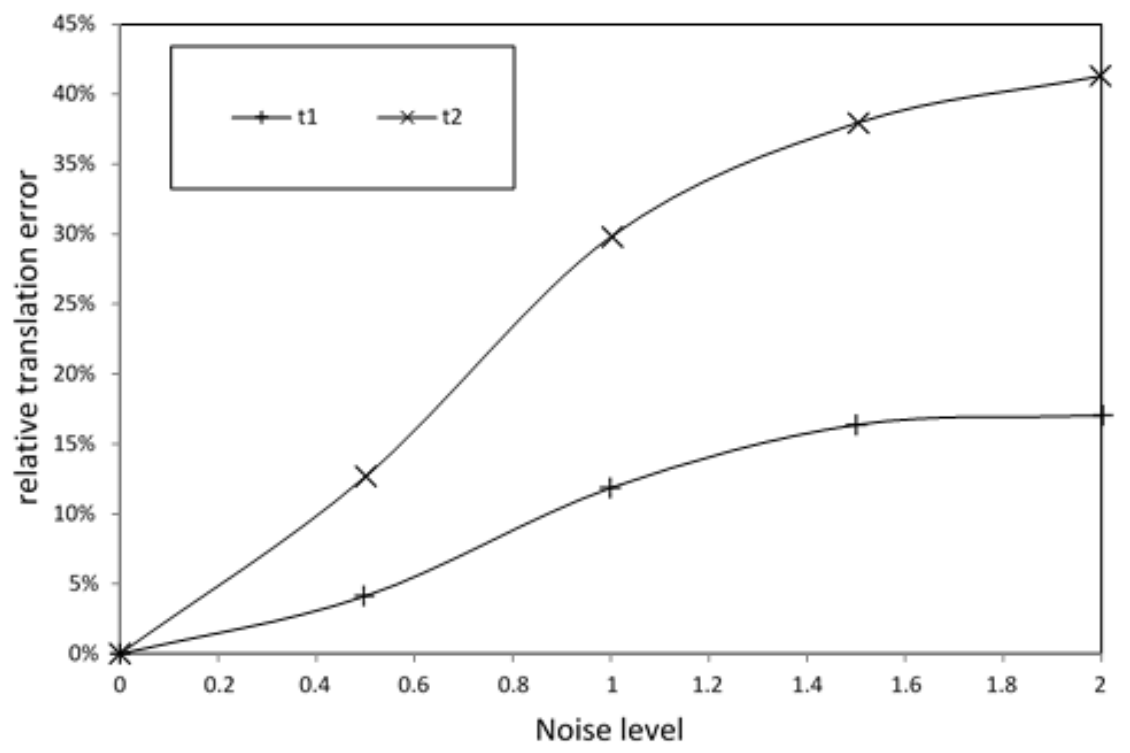

Figure 7.4: Relative translation error via noise level

\subsubsection{The Pose Measurements}

In this work, the accuracy and robustness of pose estimates is found using homography, the ICP algorithm and Horns absolute orientation method. All data are from two image views (front and top), as shown in Fig. 7.5 and 7.6, and compared with 
respect to the relative motion parameters. These algorithms are studied in Table 7.2, 7.3 and 7.4, with varying distances and angles between the cameras. The data gathered by the mobile robot is shown in Tables 7.2, and 7.3, while that collected by the flying robot is presented in Table 7.4. The distortion increases as the distance and angle increase. When Tables 7.3 and 7.4 are at the real distance $\left(\begin{array}{lll}10 & 0 & 0\end{array}\right)$ t1 is not affected, while t2 increases as the object distance increases. When the real distance becomes $(2000)$ the $t 1$ is not affected, while t2 decreases as object distance increases. When the baseline or distance increase, both t1 and t2 are affected.

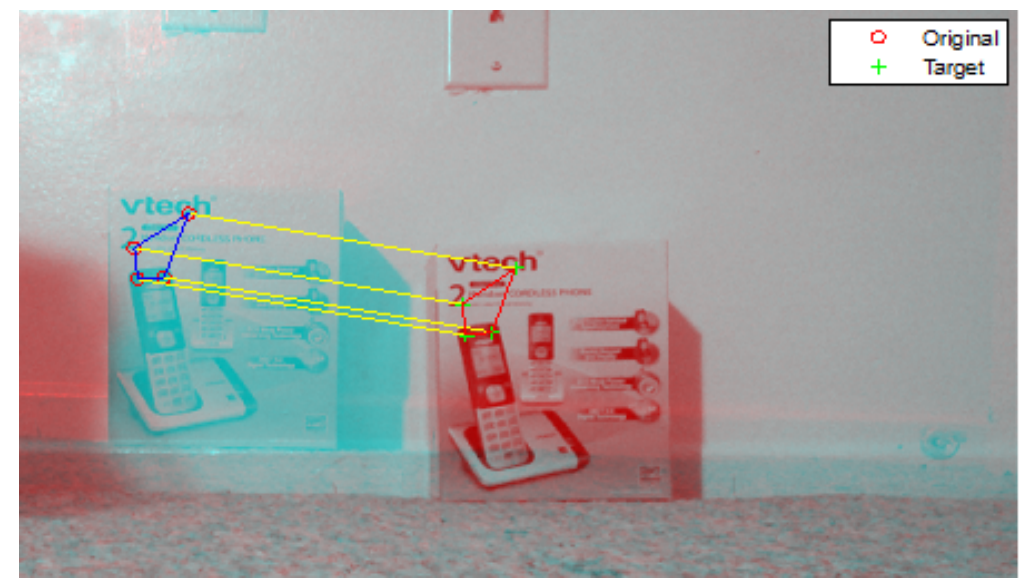

Figure 7.5: Initial and desired targets from front view

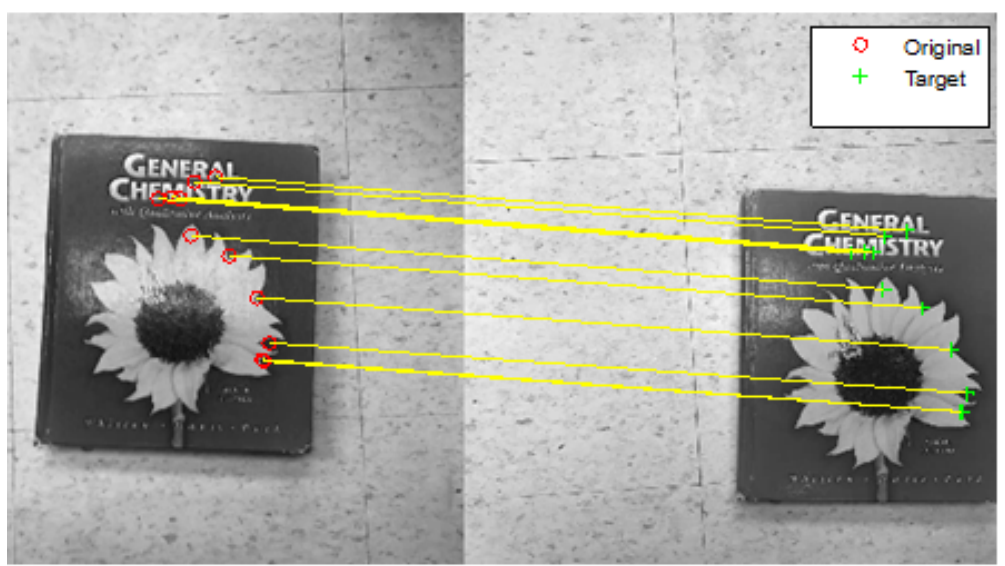

Figure 7.6: Initial and desired targets from top view 
Table 7.2: Estimation of mobile robot position for all algorithms

\begin{tabular}{|c|c|c|c|c|}
\hline \multirow[b]{2}{*}{$\begin{array}{l}\text { Object } \\
\text { distance } \\
\text { for } \mathrm{z}(\mathrm{cm})\end{array}$} & \multicolumn{4}{|c|}{ Translational,Motion $(\mathrm{t} 1, \mathrm{t} 2, \mathrm{t} 3) \mathrm{cm}$} \\
\hline & $\begin{array}{l}\text { 2D } \\
\text { Homography }\end{array}$ & ICP & $\begin{array}{l}\text { Horns } \\
\text { absolute } \\
\text { orientation method }\end{array}$ & Real \\
\hline 70 & $\left(\begin{array}{lll}10.2 & 0.88 & 0\end{array}\right)$ & $\left(\begin{array}{lll}9.66 & 0.62 & 0\end{array}\right)$ & $\left(\begin{array}{llllll}9 & 9.99 & 0.26 & 0\end{array}\right)$ & $\left(\begin{array}{lll}10 & 0 & 0\end{array}\right)$ \\
\hline 80 & $\left(\begin{array}{l}10.22 .39 \\
0\end{array}\right)$ & $\left(\begin{array}{llll}10.1 & 2.27 & 0\end{array}\right)$ & $\left(\begin{array}{lllll}10.0 & 2.50 & 0\end{array}\right)$ & 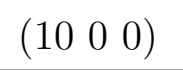 \\
\hline 90 & $\left(\begin{array}{lll}10.1 & 2.98 & 0\end{array}\right)$ & $\left(\begin{array}{llll}10.0 & 3.26 & 0\end{array}\right)$ & $\left(\begin{array}{llllll}10.1 & 3.07 & 0\end{array}\right)$ & 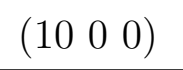 \\
\hline 150 & $(9.636 .760)$ & $(10.16 .770)$ & $\left(\begin{array}{lllllll} & 10.0 & 6.82 & 0\end{array}\right)$ & $\left(\begin{array}{lll}10 & 0 & 0\end{array}\right)$ \\
\hline 70 & $(19.83 .390)$ & $(19.85 .120)$ & $(19.83 .970)$ & 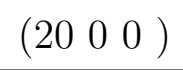 \\
\hline 80 & $(20.33 .470)$ & $\left(\begin{array}{lll}20.1 & 4.05 & 0\end{array}\right)$ & $(20.33 .430)$ & 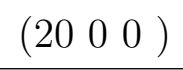 \\
\hline 90 & $(20.21 .400)$ & $\left(\begin{array}{lll}20.2 & 1.22 & 0\end{array}\right)$ & $\left(\begin{array}{lllllll}20.1 & 1.54 & 0\end{array}\right)$ & 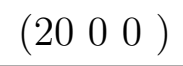 \\
\hline 150 & $\left(\begin{array}{llll}19.2 & 1.18 & 0\end{array}\right)$ & $(20.41 .160)$ & $\left(\begin{array}{lllllll}19.5 & 1.06 & 0\end{array}\right)$ & 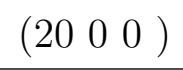 \\
\hline 150 & $(0.175 .220)$ & $\left(\begin{array}{lll}0.76 & 4.23 & 0\end{array}\right)$ & $(0.125 .400)$ & $\left(\begin{array}{lll}0 & 5 & 0\end{array}\right)$ \\
\hline 150 & $(22.66 .000)$ & $(23.05 .500)$ & $(22.86 .200)$ & $\left(\begin{array}{lll}20 & 10 & 0\end{array}\right)$ \\
\hline 150 & $(19.73 .580)$ & $\left(\begin{array}{lll}19.7 & 2.65 & 0\end{array}\right)$ & (19.7 3.05 0) & $(2050)$ \\
\hline 300 & $(28.42 .100)$ & $\left(\begin{array}{lll}28.6 & 1.50 & 0\end{array}\right)$ & $(27.43 .050)$ & 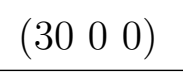 \\
\hline 500 & $\left(\begin{array}{llll}37.8 & 3.30 & 0\end{array}\right)$ & $\left(\begin{array}{lll}38.1 & 3.10 & 0\end{array}\right)$ & $(36.64 .200)$ & $\left(\begin{array}{lll}4 & 0 & 0\end{array}\right)$ \\
\hline 1000 & $\left(\begin{array}{lll}45.5 & 4.30 & 0\end{array}\right)$ & $\left(\begin{array}{lll}46.3 & 4.01 & 0\end{array}\right)$ & $(44.74 .500)$ & 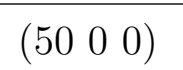 \\
\hline
\end{tabular}


Table 7.3: Estimation of mobile robot position for two baselines

\begin{tabular}{|c|c|c|c|}
\hline & \multicolumn{3}{|c|}{ Baseline $=10 \mathrm{~cm}$} \\
\hline $\begin{array}{l}\text { Object } \\
\text { distance } \\
\text { for } \mathrm{z}(\mathrm{cm})\end{array}$ & $\begin{array}{l}\text { 2D } \\
\text { Homography }\end{array}$ & ICP & $\begin{array}{l}\text { Horns } \\
\text { absolute } \\
\text { orientation method }\end{array}$ \\
\hline 70 & $\left(\begin{array}{lll}10.2 & 0.88 & 0\end{array}\right)$ & $\left(\begin{array}{lll}9.66 & 0.62 & 0\end{array}\right)$ & $\left(\begin{array}{lll}9.99 & 0.26 & 0\end{array}\right)$ \\
\hline 80 & $\left(\begin{array}{l}10.2 \\
2.39\end{array}\right)$ & (10.1 2.27 0) & $\left(\begin{array}{llll}10.0 & 2.50 & 0\end{array}\right)$ \\
\hline 90 & $\left(\begin{array}{lll}10.1 & 2.98 & 0\end{array}\right)$ & $\left(\begin{array}{llll}10.0 & 3.26 & 0\end{array}\right)$ & $\left(\begin{array}{llll}10.1 & 3.07 & 0\end{array}\right)$ \\
\hline \multirow[t]{2}{*}{150} & $\left(\begin{array}{llll}9.63 & 6.76 & 0\end{array}\right)$ & (10.1 6.77 0) & (10.0 6.820$)$ \\
\hline & \multicolumn{3}{|c|}{ Baseline $=20 \mathrm{~cm}$} \\
\hline 70 & (19.8 3.390$)$ & (19.8 5.12 0) & (19.8 3.970$)$ \\
\hline 80 & $(20.33 .470)$ & $(20.14 .050)$ & $(20.33 .430)$ \\
\hline 90 & $\left(\begin{array}{lll}20.2 & 1.40 & 0\end{array}\right)$ & $\left(\begin{array}{lll}20.2 & 1.22 & 0\end{array}\right)$ & $\left(\begin{array}{llll}20.1 & 1.54 & 0\end{array}\right)$ \\
\hline 150 & $\left(\begin{array}{lll}19.2 & 1.18 & 0\end{array}\right)$ & $\left(\begin{array}{lll}20.4 & 1.16 & 0\end{array}\right)$ & $\left(\begin{array}{lll}19.5 & 1.06 & 0\end{array}\right)$ \\
\hline
\end{tabular}

Table 7.4: Estimation of flying robot position for two baselines

\begin{tabular}{|c|c|c|c|}
\hline & \multicolumn{3}{|c|}{ Baseline $=10 \mathrm{~cm}$} \\
\hline $\begin{array}{l}\text { Object } \\
\text { distance } \\
\text { for } \mathrm{z}(\mathrm{cm})\end{array}$ & $\begin{array}{l}\text { 2D } \\
\text { Homography }\end{array}$ & ICP & $\begin{array}{l}\text { Horns } \\
\text { absolute } \\
\text { orientation method }\end{array}$ \\
\hline 60 & $\left(\begin{array}{lll}10.2 & 0.33 & 0\end{array}\right)$ & $\left(\begin{array}{lll}9.80 & 0.32 & 0\end{array}\right)$ & $\left(\begin{array}{lll}10.1 & 0.56 & 0\end{array}\right)$ \\
\hline 80 & (10.2 3.130$)$ & (10.1 2.150$)$ & (10.1 3.100$)$ \\
\hline 120 & (10.3 3.190$)$ & (10.2 3.700$)$ & (10.1 3.26 0) \\
\hline \multirow[t]{2}{*}{150} & 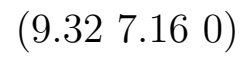 & (10.2 5.870$)$ & (10.1 7.14 0) \\
\hline & \multicolumn{3}{|c|}{ Baseline $=20 \mathrm{~cm}$} \\
\hline 60 & (19.6 2.110$)$ & (19.5 4.320$)$ & (19.98 3.20$)$ \\
\hline 80 & $\left(\begin{array}{llll}20.4 & 4.13 & 0\end{array}\right)$ & 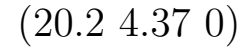 & (20.4 4.300$)$ \\
\hline 120 & $\left(\begin{array}{llll}20.4 & 3.50 & 0\end{array}\right)$ & $\left(\begin{array}{lll}20.3 & 2.41 & 0\end{array}\right)$ & (20.2 2.660$)$ \\
\hline 150 & (19.4 2.80 0) & $\left(\begin{array}{lll}20.5 & 2.70 & 0\end{array}\right)$ & (19.5 2.600$)$ \\
\hline
\end{tabular}




\subsection{Results of the Flexible Wing}

The proposed DDVS for flexible wing model in this thesis is shown in Fig. 7.7. The process begins by capturing images using the ZED stereo camera, then extracting the features with the SURF method. After the 3D point is computed for each point with machine vision, the shape of the wing is identified as described in Chapter 3.

The shape is classified by fuzzy logic to determine the state of the system such as pitch angle, speed, and roll angle. Sensor fusion is used to estimate the state of the flexible wing by fusing the measurement states from DDVS with other sensor measurements. After the speed, pitch and roll angles are estimated, the errors from the desired and estimated state are input for the longitudinal and lateral autopilot controllers. These controllers are designed to control the flexible wing UAV based on ANFIS and PID controllers consider the disturbance in all states of the system.

In this thesis, experimental testing is performed to clarify the proposed approach to deflection measurement of a flexible wing using a stereo camera. The UAV platform used in wind tunnel testing has flexible $100 \mathrm{~cm}$ long wings with a $120 \mathrm{~cm}$ wingspan, and carries no internal components such as a fuselage or engine. The wing is composed of a plastic fiber and the body structure is made of wood. One advantages of flexible wings is they have greater resistance at high AOA, as well as increased roll stability. Six square markers attached to the end of the wing are used to gather the measurement data from the stereo camera as shown in Fig. 7.8, 7.9 and Fig. 7.10. 


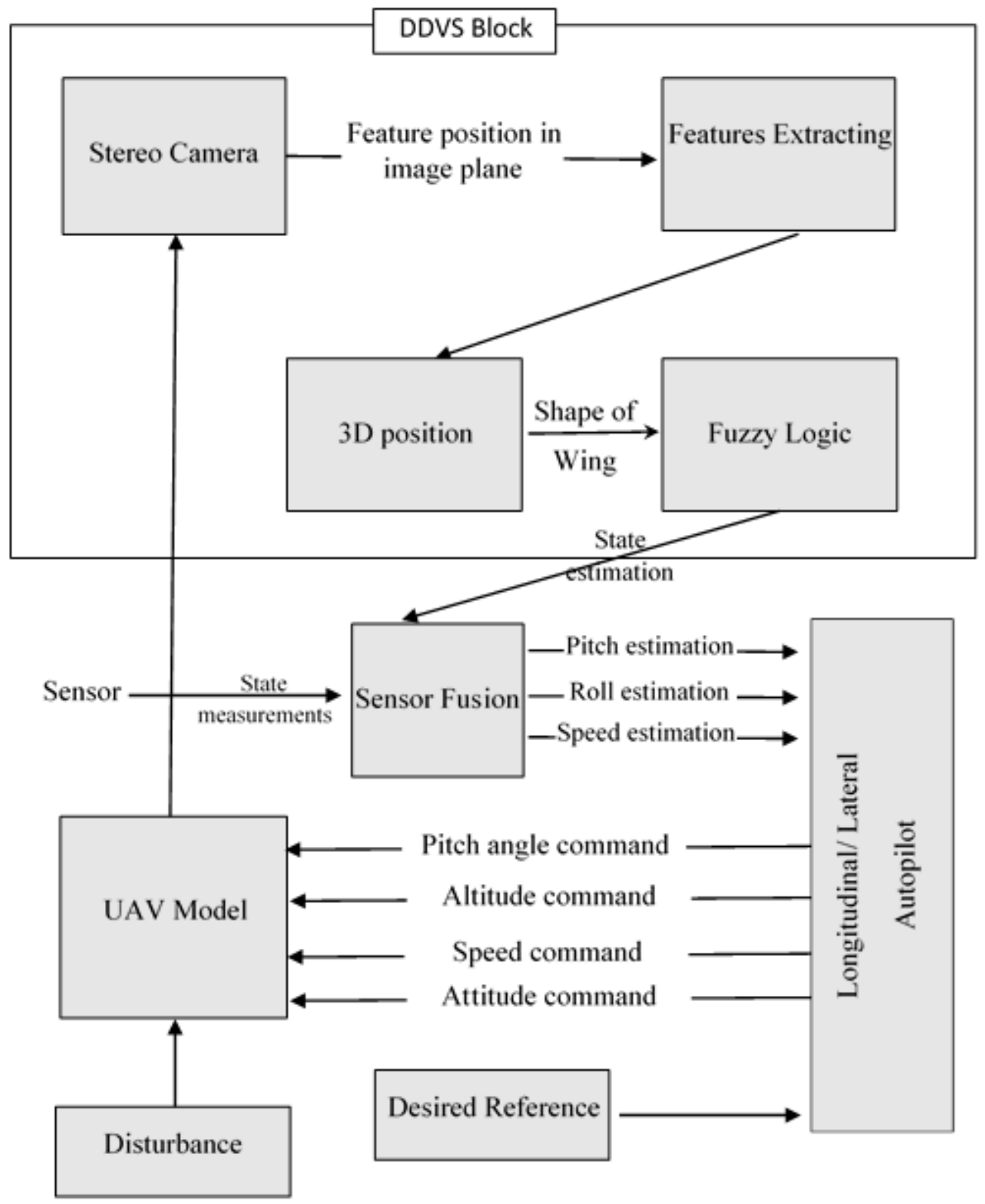

Figure 7.7: The Detection-Deflection Vision System(DDVS) for the flexible wing model

\subsubsection{Wing Shape}

The ZED stereo camera is attached behind the wing on the keel tube to ensure the wing is in the view and can provide vision data of the square markers. The testing 


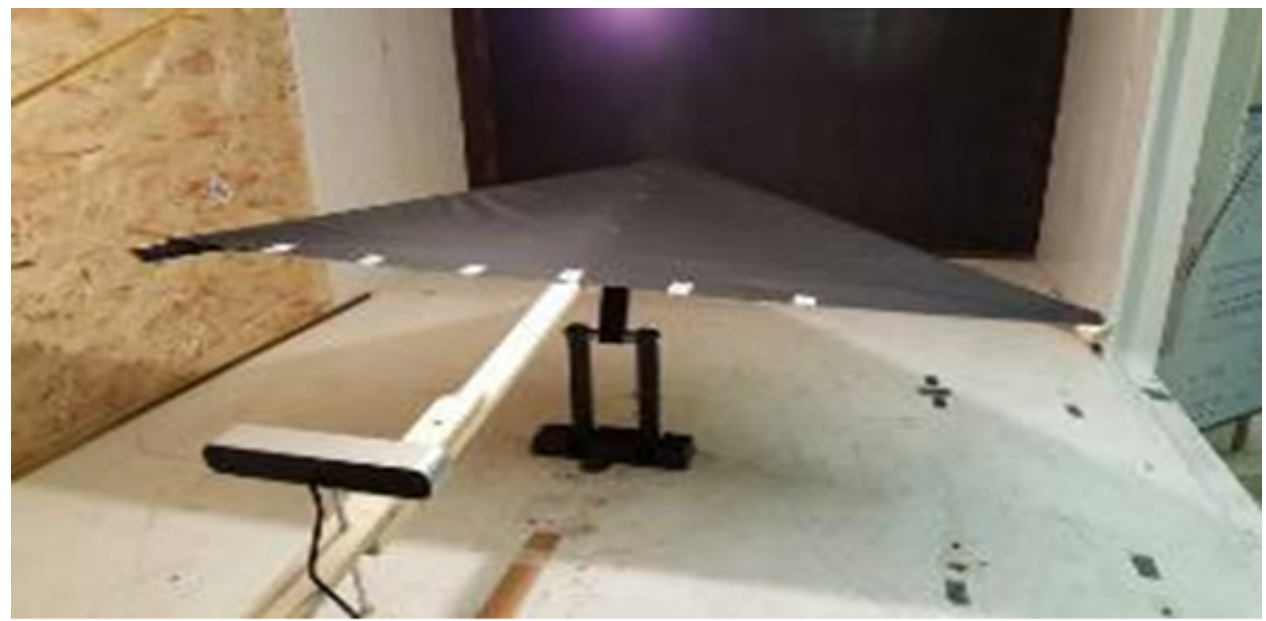

Figure 7.8: Back view of the flexible wing in wind tunnel

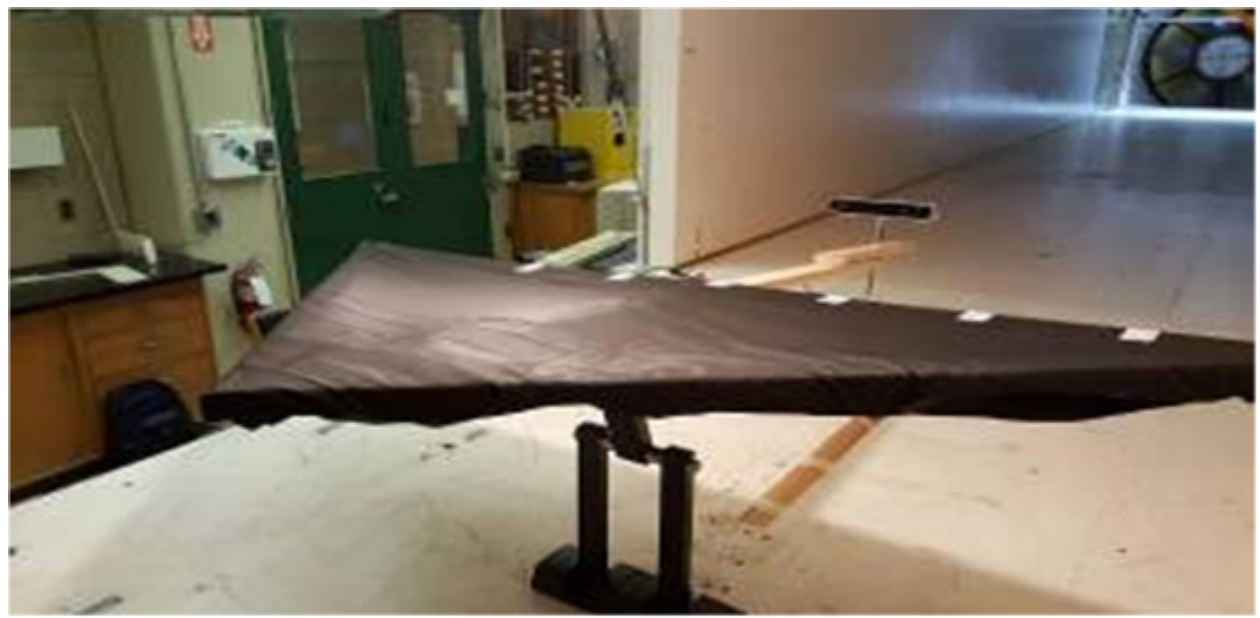

Figure 7.9: Front view of the flexible wing in wind tunnel

begins by measuring the deflection of the wing using the two left and right images. Pictures of the wing are taken at $\mathrm{AOA}=0^{0}$, and roll angle $=0^{0}$, with specific wind velocities $(11$ to 31$) \mathrm{m} / \mathrm{s}$ as shown in Fig. 7.11. The AOA value of the wing is then increased to $+20^{0}$, the roll angle is unchanged and pictures are taken at the same wind velocity values, as shows in Fig. 7.12, 7.13, and 7.14. The testing is repeated with the same conditions when the AOA reaches $-20^{\circ}$, as shown in Fig. 7.15 , then the roll angle is changed from zero to $10^{\circ}$ and AOA to $20^{\circ}$ with the same wind velocities, as shown in Fig. 7.16. Finally, the roll angle is set to $-10^{\circ}$ and AOA to $-20^{\circ}$ and 


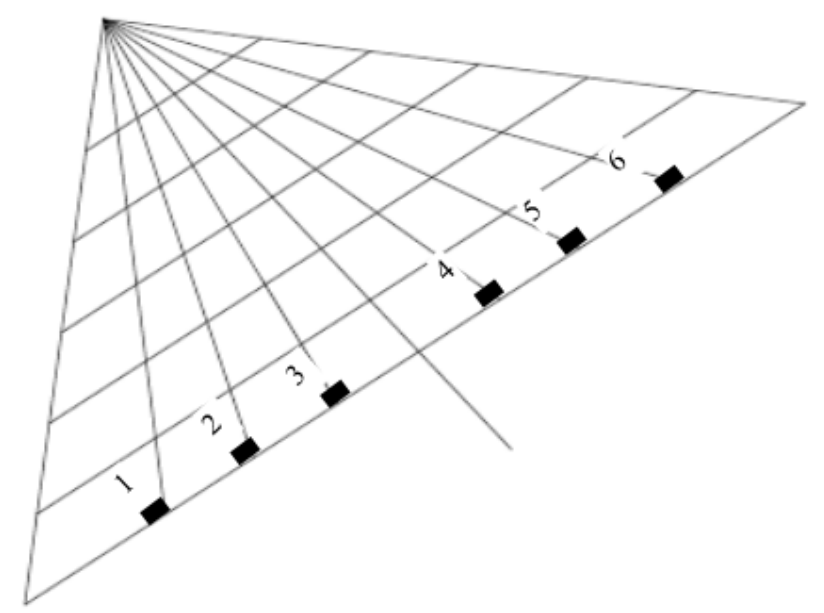

Figure 7.10: Features position

pictures are taken for different wind values, as shown in Fig. 7.17.

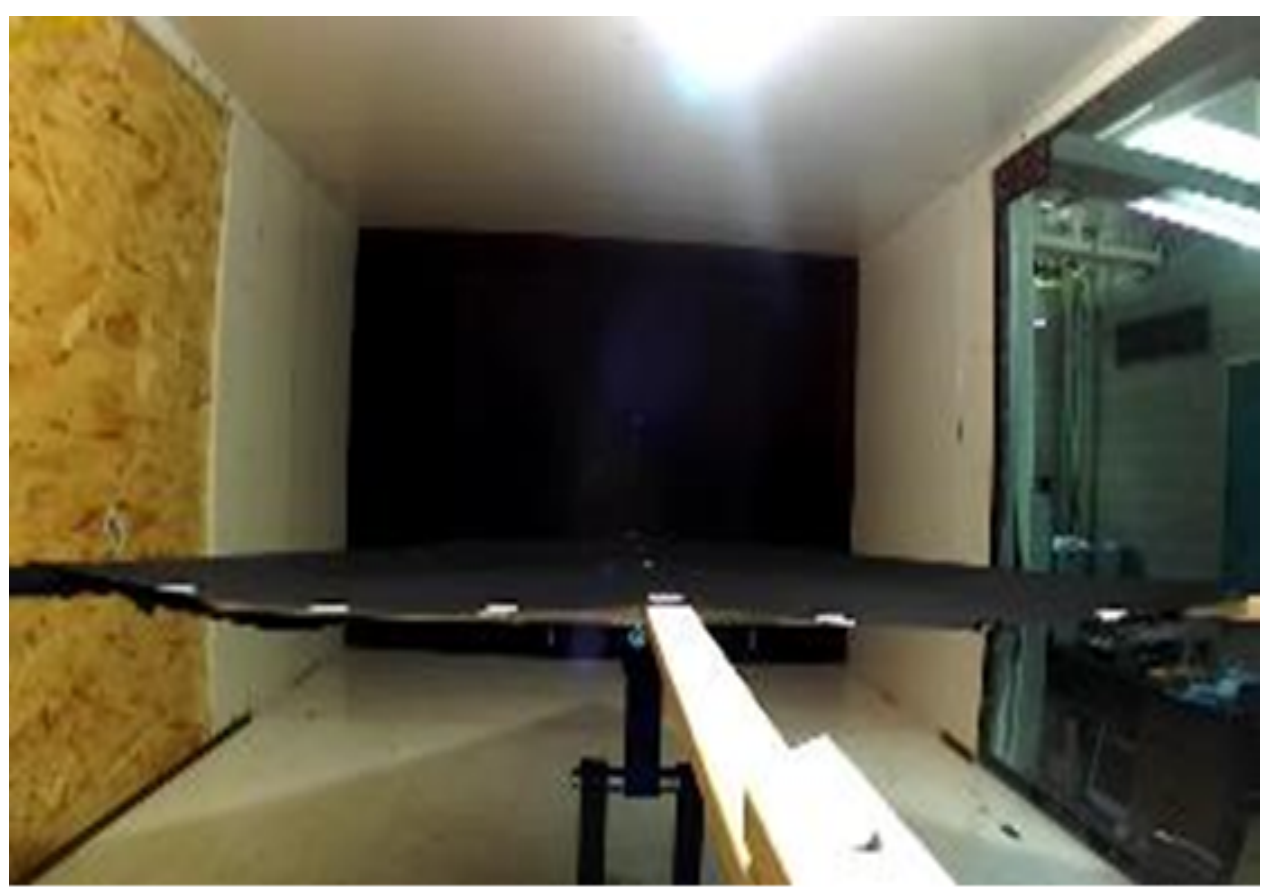

Figure 7.11: Shape of wing at $V=31 \mathrm{~m} / \mathrm{s}$ 


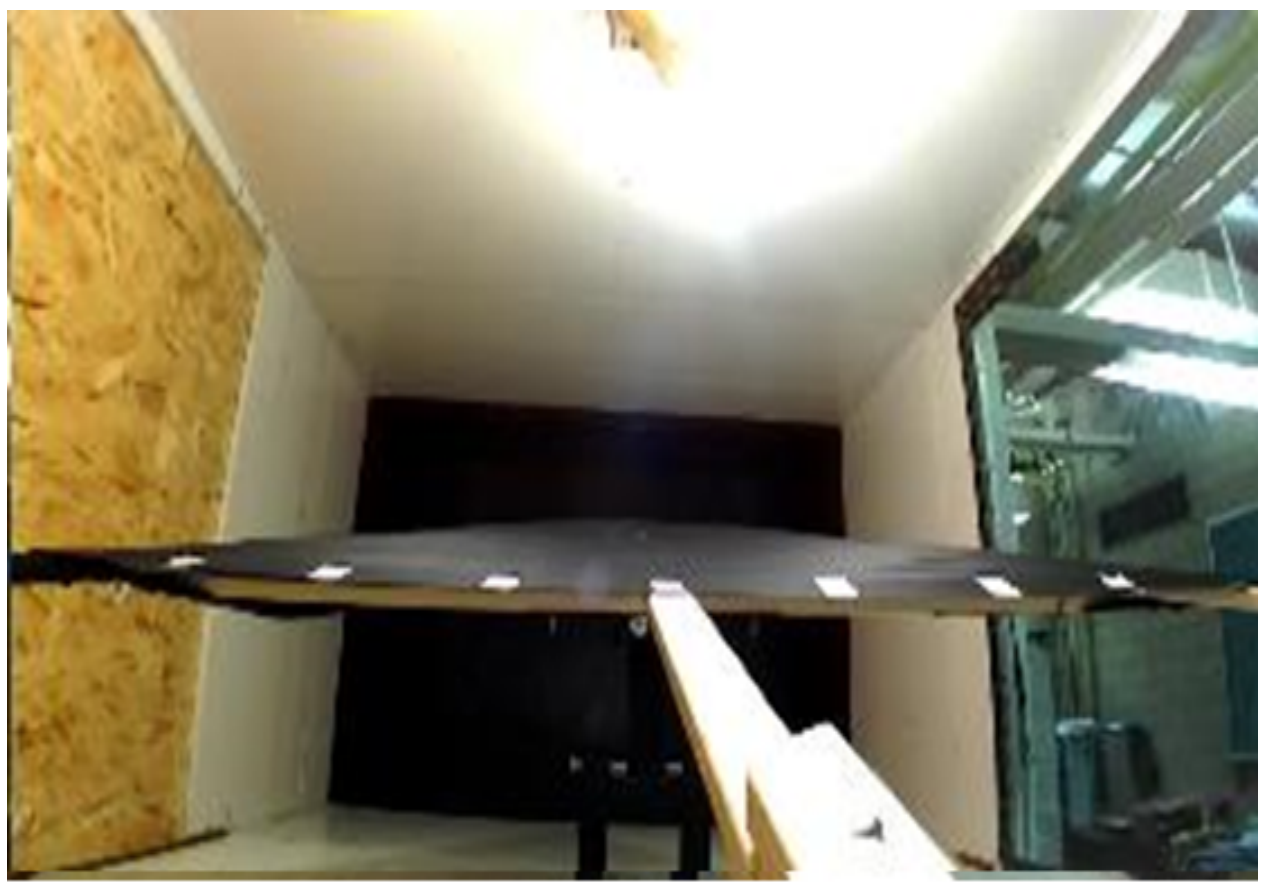

Figure 7.12: Shape of wing at $\mathrm{V}=11 \mathrm{~m} / \mathrm{s}$ and $\mathrm{AOA}=+20^{0}$

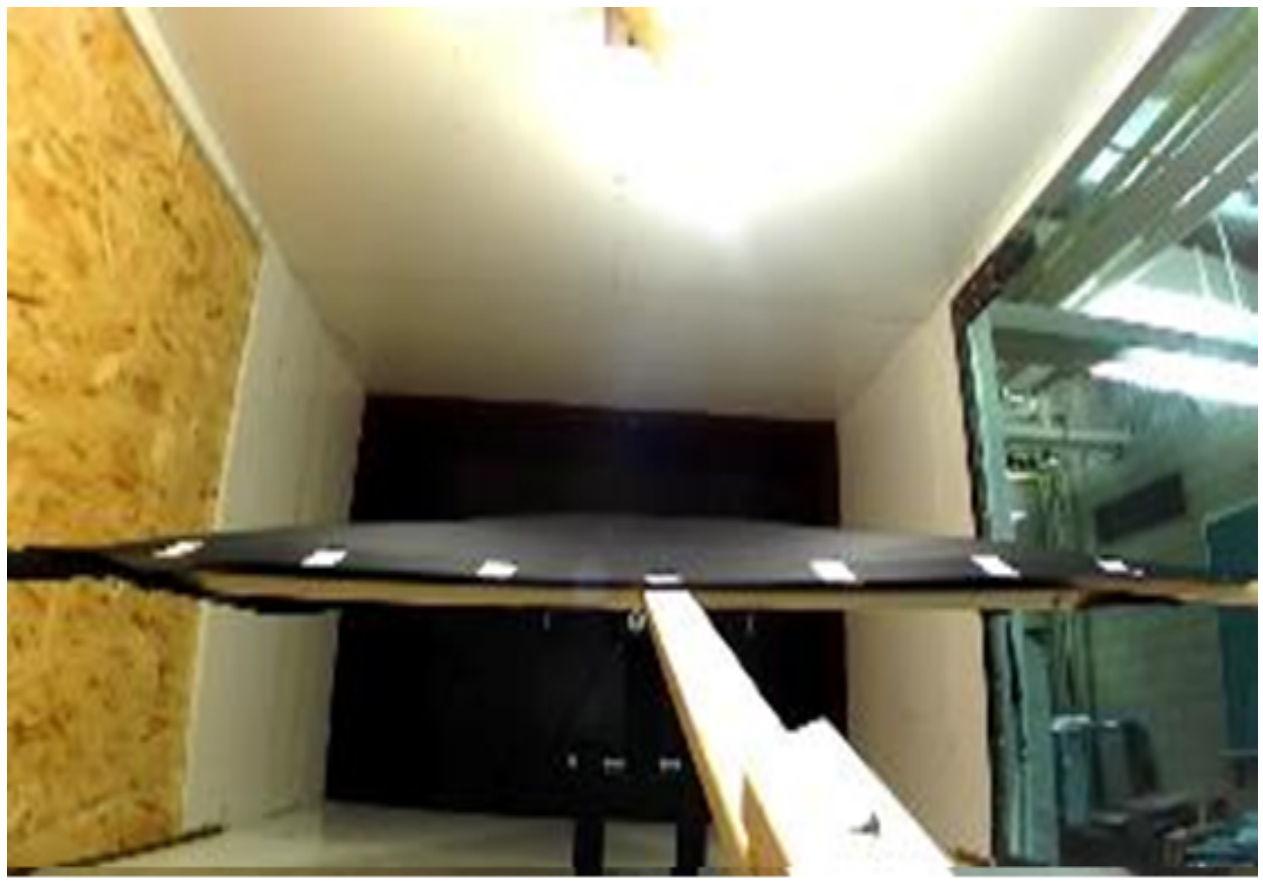

Figure 7.13: Shape of wing at $\mathrm{V}=21 \mathrm{~m} / \mathrm{s}$ and $\mathrm{AOA}=+20^{0}$ 


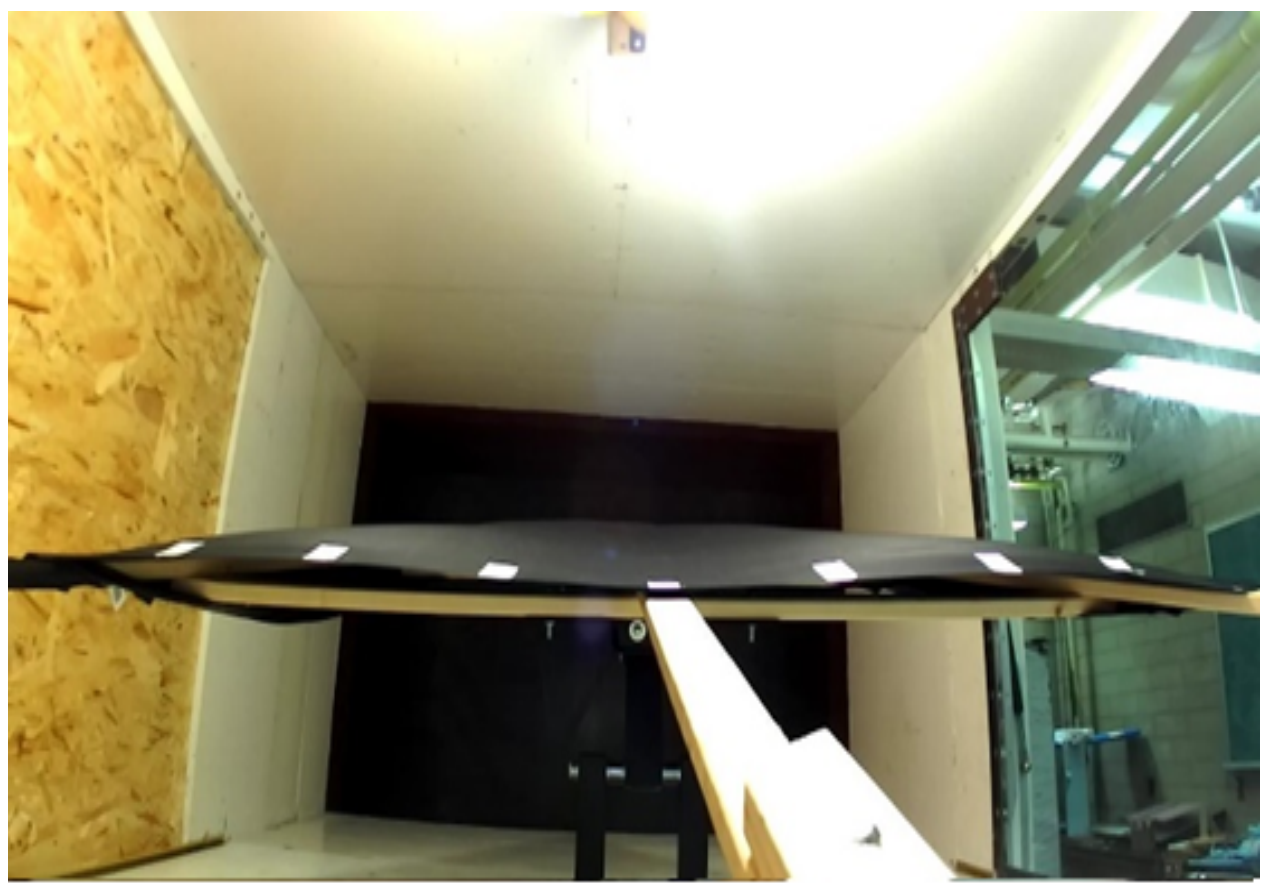

Figure 7.14: Shape of wing at $\mathrm{V}=31 \mathrm{~m} / \mathrm{s}$ and $\mathrm{AOA}=+20^{\circ}$

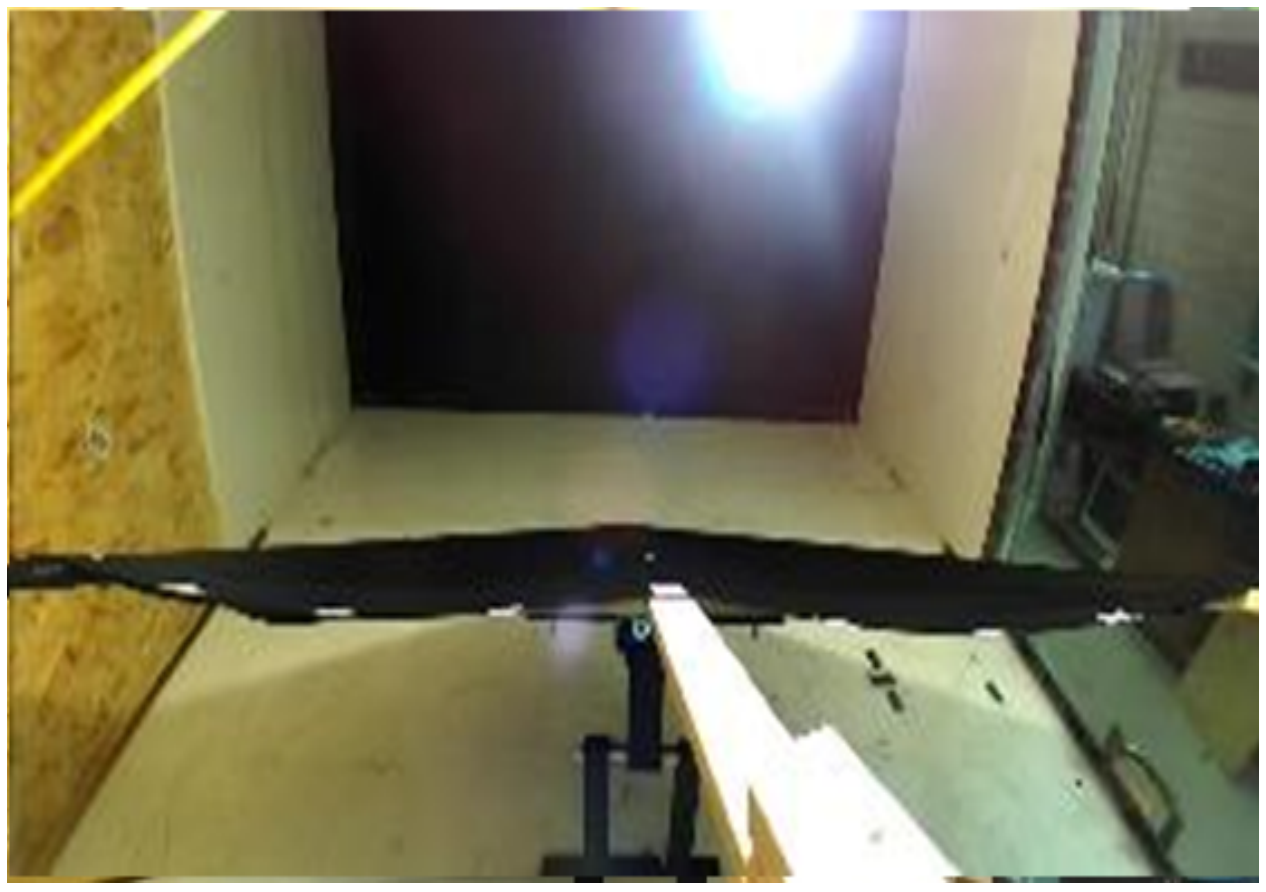

Figure 7.15: Shape of wing at $\mathrm{V}=31 \mathrm{~m} / \mathrm{s}$ and $\mathrm{AOA}=-20^{\circ}$ 


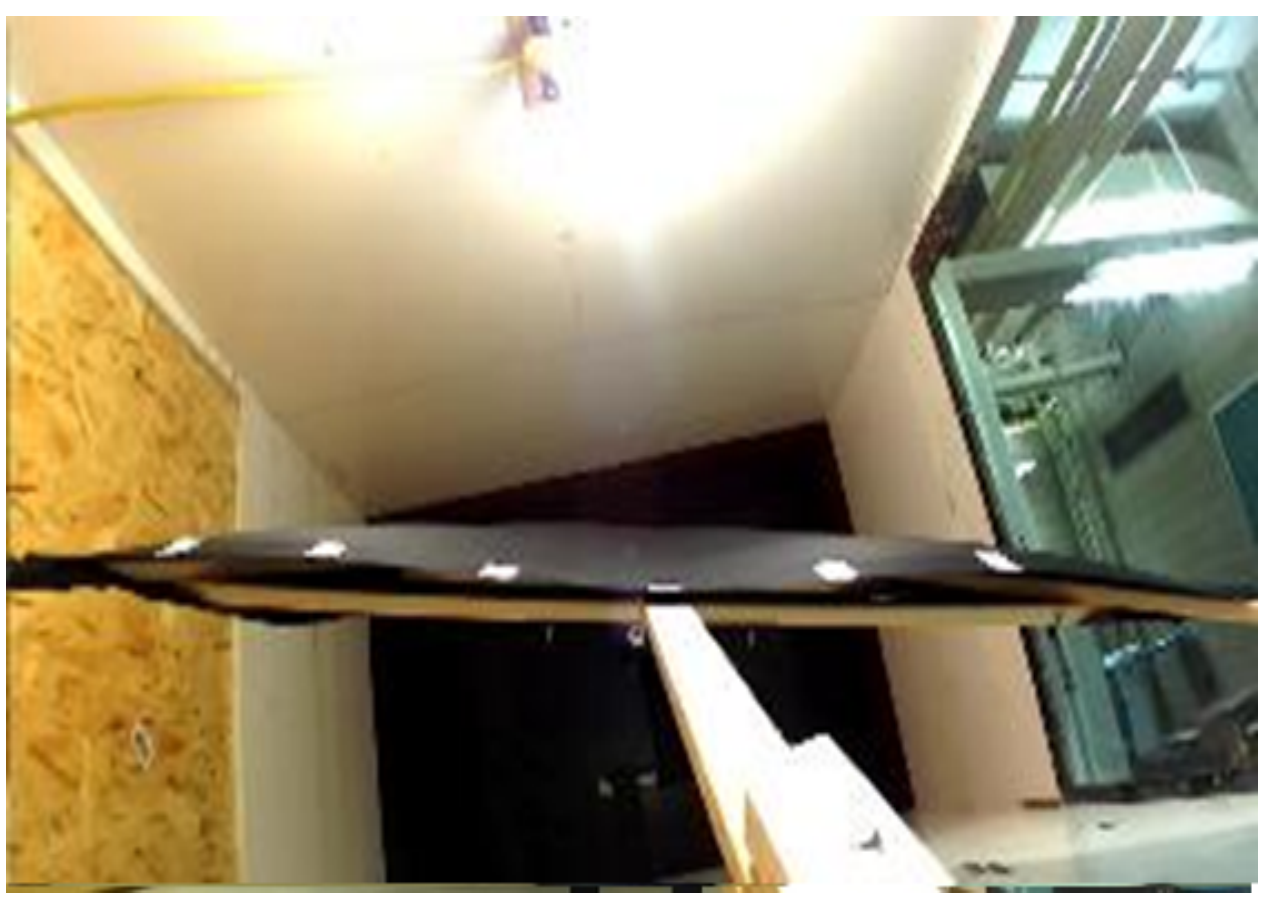

Figure 7.16: Shape of wing at $\mathrm{V}=31 \mathrm{~m} / \mathrm{s}$, Roll angle $=10^{\circ}$ and $\mathrm{AOA}=+20^{0}$

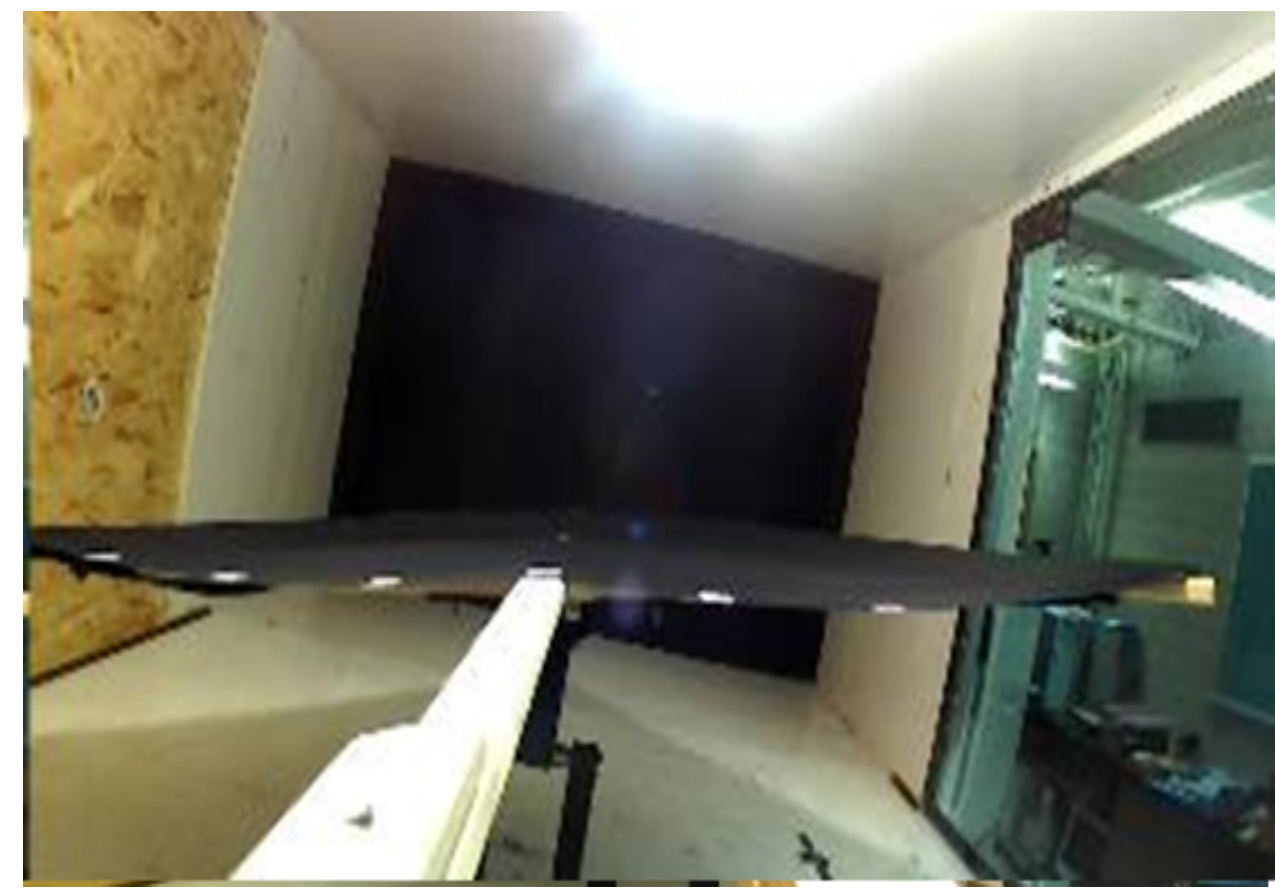

Figure 7.17: Shape of wing at $V=11 \mathrm{~m} / \mathrm{s}$, Roll angle $=-10^{\circ}$ and $\mathrm{AOA}=-20^{\circ}$ 


\subsubsection{Feature Extraction}

In this subsection, a stereo camera is used to generate multiple images and extract the most relevant features. The process starts by selecting a set of interest points and constructing their local feature descriptors using SURF. Then, representative points from the interest points are founded by using statistical analysis. The representative points of an object are interest points that distinguish information about an object, as shown in Fig. 7.18.

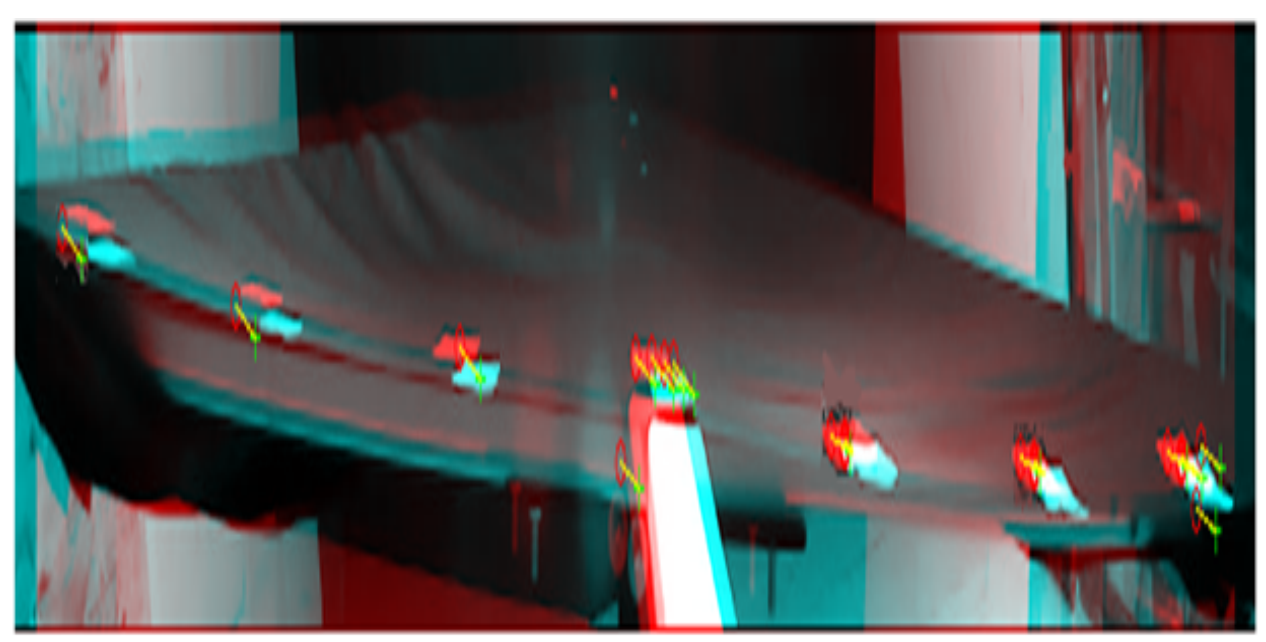

Figure 7.18: The matching of the feature points

\subsubsection{Extract Deflection using the Stereo Camera}

Several shapes of the flexible wing are observed in the wind tunnel. Once the wing is loaded, its deflected profile (shape) is computed using the six markers shown in Fig. 7.10. At $\mathrm{AOA}=0$ and roll angle $=0$, five shapes of the wing across variety speed are shown in Fig. 7.19. The deflection is changed depending on the speed, the maximum deflection is $-0.14 \mathrm{~cm}$ when the wind speed reached $31 \mathrm{~m} / \mathrm{s}$. The shape of the wing was symmetrical, and the peak deflection occurred at mid-distance of each side of the 
wing as shwon in Fig. 7.19.

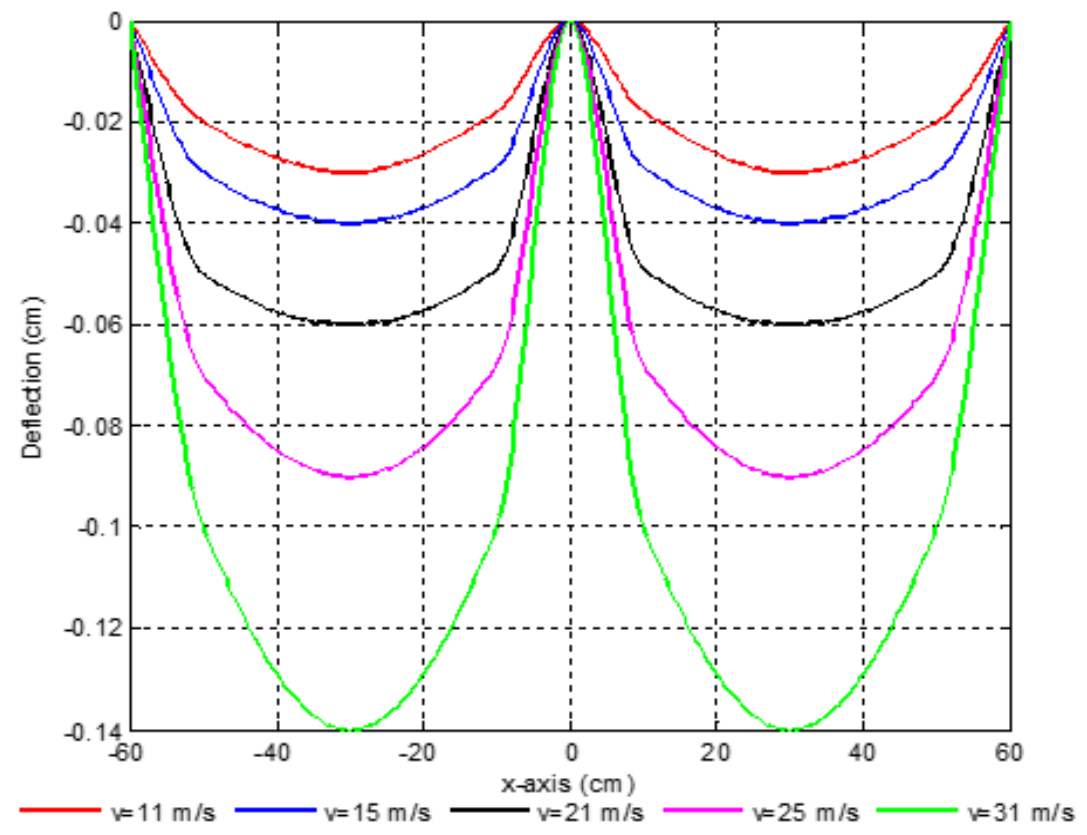

Figure 7.19: Shape of wing at $\mathrm{AOA}=0^{0}$ and roll angle $=0^{0}$

When the AOA is $-20^{\circ}$, the maximum deflection increased to $-0.5 \mathrm{~cm}$, and the shape of the wing is also symmetrical on both sides as shown in Fig. 7.20. In Fig. 7.21 and 7.22 , the roll angle become $-10^{\circ}$ for both the $\mathrm{AOA}=0$ or the $\mathrm{AOA}=-20^{\circ}$. The change in roll angle makes the wing shape not symmetrical on both sides. The same result form roll angle change when the $\mathrm{AOA}=20^{\circ}$, as shown in Fig. 7.23 except the maximum deflection in the positive value. From observation, the greatest deflection occurred at the AOA is $20^{\circ}$, as shown in Fig. 7.24. 


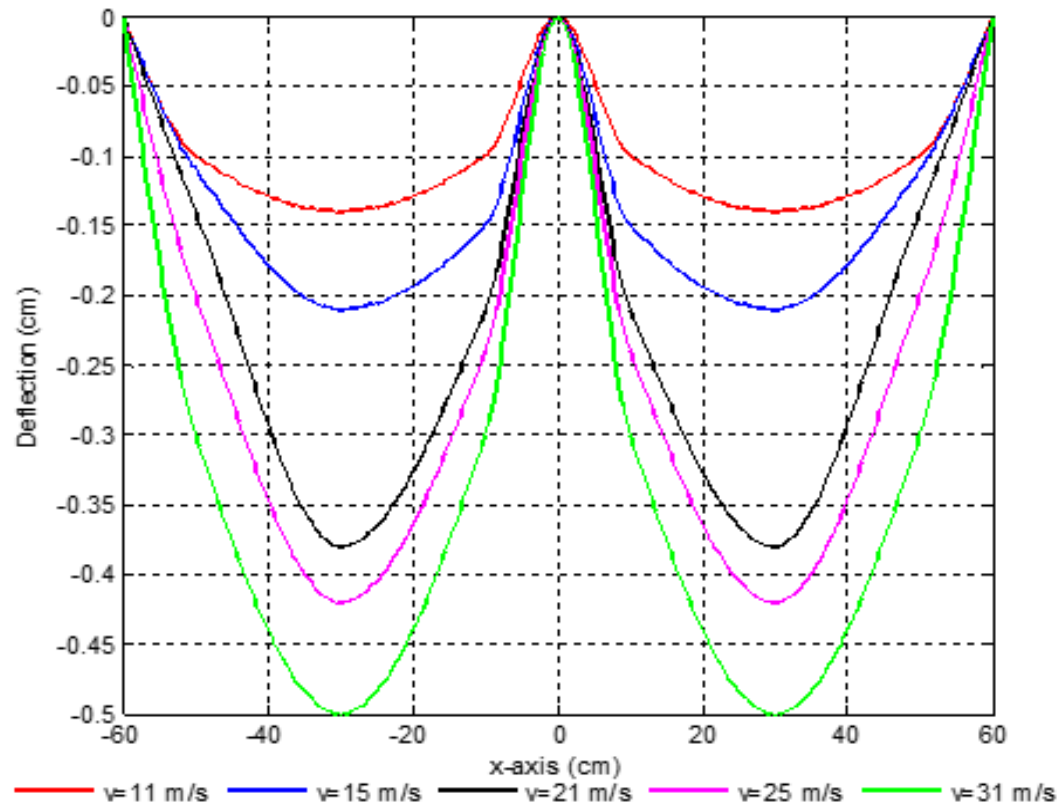

Figure 7.20: Shape of wing at $\mathrm{AOA}=-20^{0}$ and roll angle $=0^{0}$

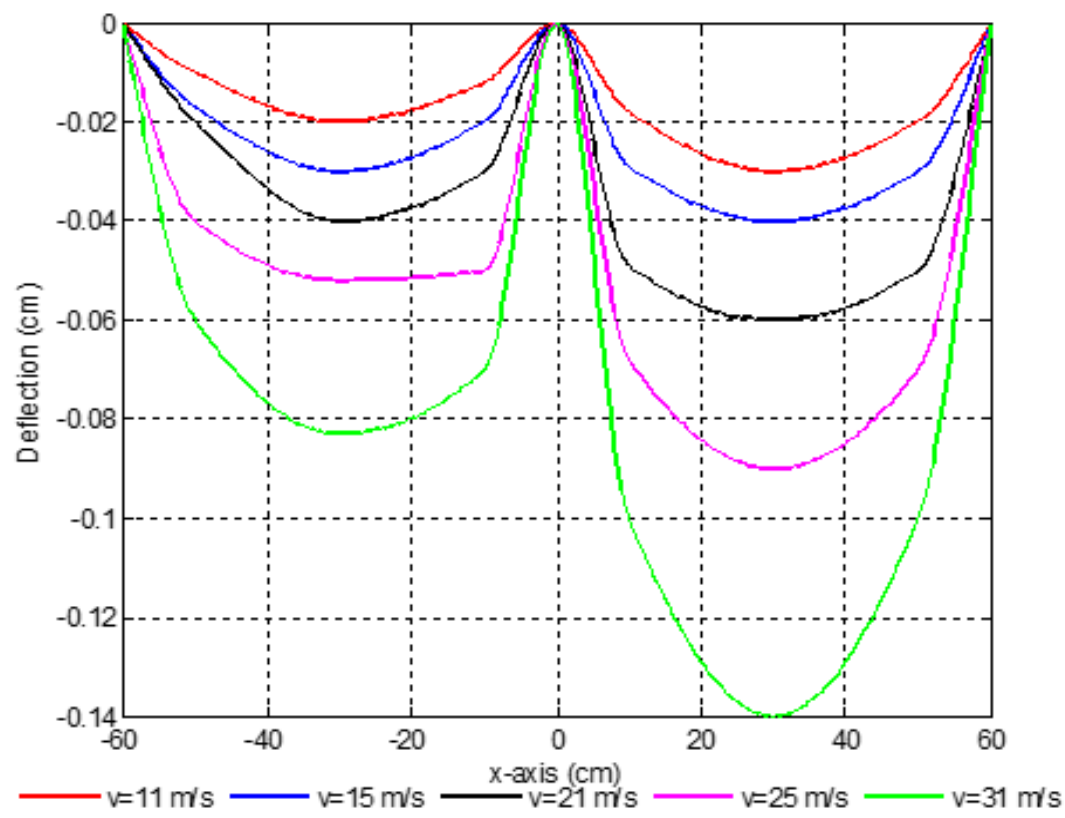

Figure 7.21: Shape of wing at $\mathrm{AOA}=0^{0}$ and roll angle $=-10^{0}$ 


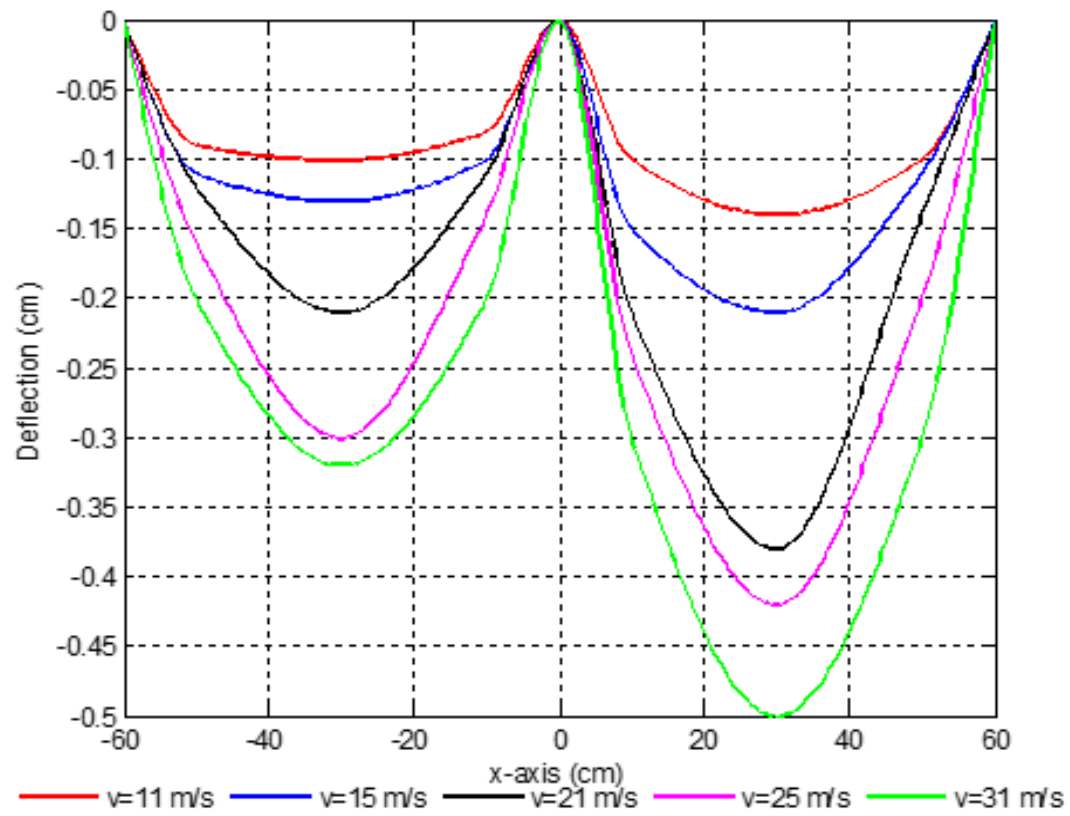

Figure 7.22: Shape of wing at $\mathrm{AOA}=-20^{\circ}$ and roll angle $=-10^{0}$

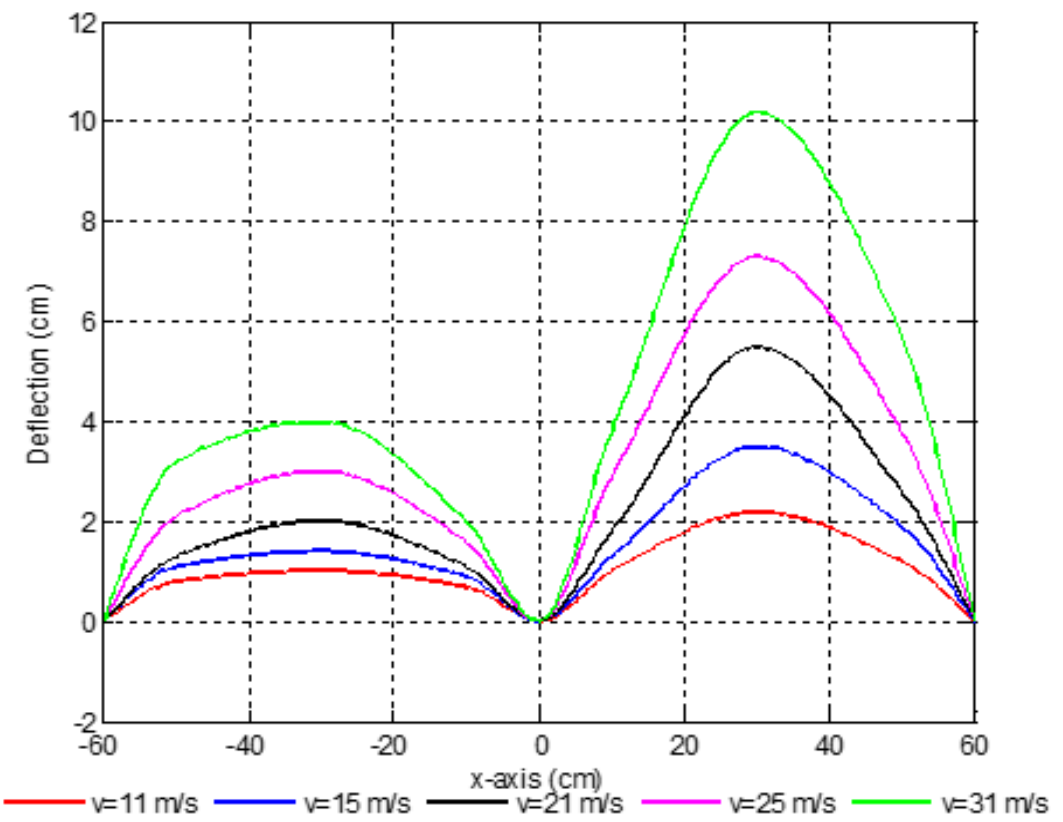

Figure 7.23: Shape of wing at $\mathrm{AOA}=20^{\circ}$ and roll angle $=-10^{0}$ 


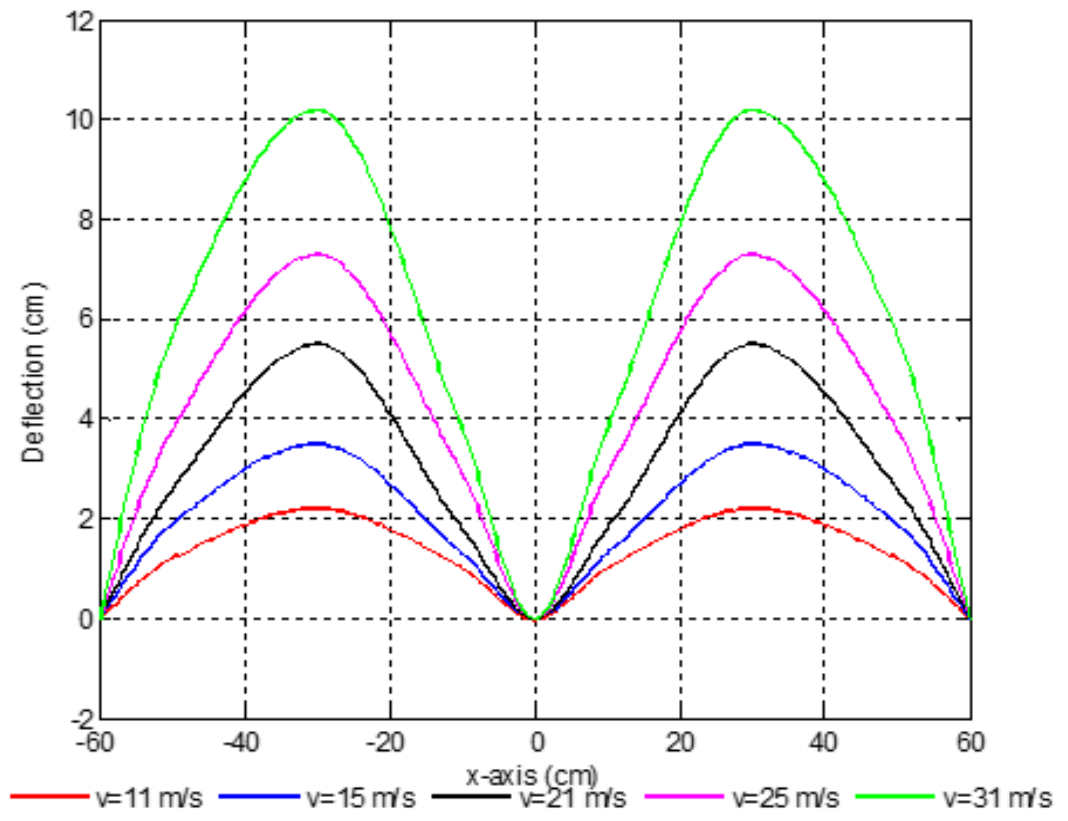

Figure 7.24: Shape of wing at $\mathrm{AOA}=20^{\circ}$ and roll angle $=0^{0}$

Table 7.5 and 7.6 show the maximum deflection of the flexible wing for each test cases. This table helps to clarify the accuracy of DDVS algorithm.

The dynamic effect on each point is shown in Fig. 7.25, and the deflection is negative values at the start experimental until to $50 \mathrm{sec}$, then tends to zero until 100 sec, finally, the deflection increases to positive values after $100 \mathrm{sec}$. The reason is at the start the AOA is -20 and after 50 sec close to zero, and after $100 \mathrm{sec}$ the AOA is $+20^{0}$. 
Table 7.5: Test Results for AOA

\begin{tabular}{|c|c|c|}
\hline $\mathrm{AOA}(\mathrm{deg})$ & Speed $(\mathrm{m} / \mathrm{s})$ & Max. deflection $(\mathrm{cm})$ \\
\hline \multirow{5}{*}{-20} & 11 & -0.14 \\
\hline & 15 & -0.21 \\
\hline & 21 & -0.38 \\
\hline & 25 & -0.42 \\
\hline & 31 & -0.50 \\
\hline \multirow{5}{*}{-10} & 11 & -0.07 \\
\hline & 15 & -0.12 \\
\hline & 21 & -0.18 \\
\hline & 25 & -0.36 \\
\hline & 31 & -0.31 \\
\hline \multirow{5}{*}{0} & 11 & -0.03 \\
\hline & 15 & -0.04 \\
\hline & 21 & -0.06 \\
\hline & 25 & -0.09 \\
\hline & 31 & -0.14 \\
\hline \multirow{5}{*}{10} & 11 & 1.42 \\
\hline & 15 & 1.91 \\
\hline & 21 & 3.16 \\
\hline & 25 & 4.76 \\
\hline & 31 & 6.33 \\
\hline \multirow{5}{*}{20} & 11 & 2.28 \\
\hline & 15 & 3.53 \\
\hline & 21 & 5.51 \\
\hline & 25 & 7.33 \\
\hline & 31 & 10.3 \\
\hline
\end{tabular}


Table 7.6: Test Results for Roll angle

\begin{tabular}{|l|l|l|}
\hline Roll angle $(\mathrm{deg})$ & Speed $(\mathrm{m} / \mathrm{s})$ & Max. deflection $(\mathrm{cm})$ \\
\hline \multirow{4}{*}{-10} & 11 & 2.28 \\
\cline { 2 - 3 } & 15 & 3.53 \\
\cline { 2 - 3 } & 21 & 5.51 \\
\cline { 2 - 3 } & 25 & 7.33 \\
\cline { 2 - 3 } & 31 & 10.3 \\
\hline \multirow{5}{*}{0} & 11 & -0.03 \\
\cline { 2 - 3 } & 15 & -0.04 \\
\hline \multirow{5}{*}{10} & 21 & -0.06 \\
\cline { 2 - 3 } & 25 & -0.09 \\
\cline { 2 - 3 } & 31 & -0.14 \\
\hline \multirow{5}{*}{} & 11 & 2.28 \\
\cline { 2 - 3 } & 15 & 3.53 \\
\cline { 2 - 3 } & 21 & 5.51 \\
\cline { 2 - 3 } & 25 & 7.33 \\
\cline { 2 - 3 } & 31 & 10.3 \\
\hline
\end{tabular}



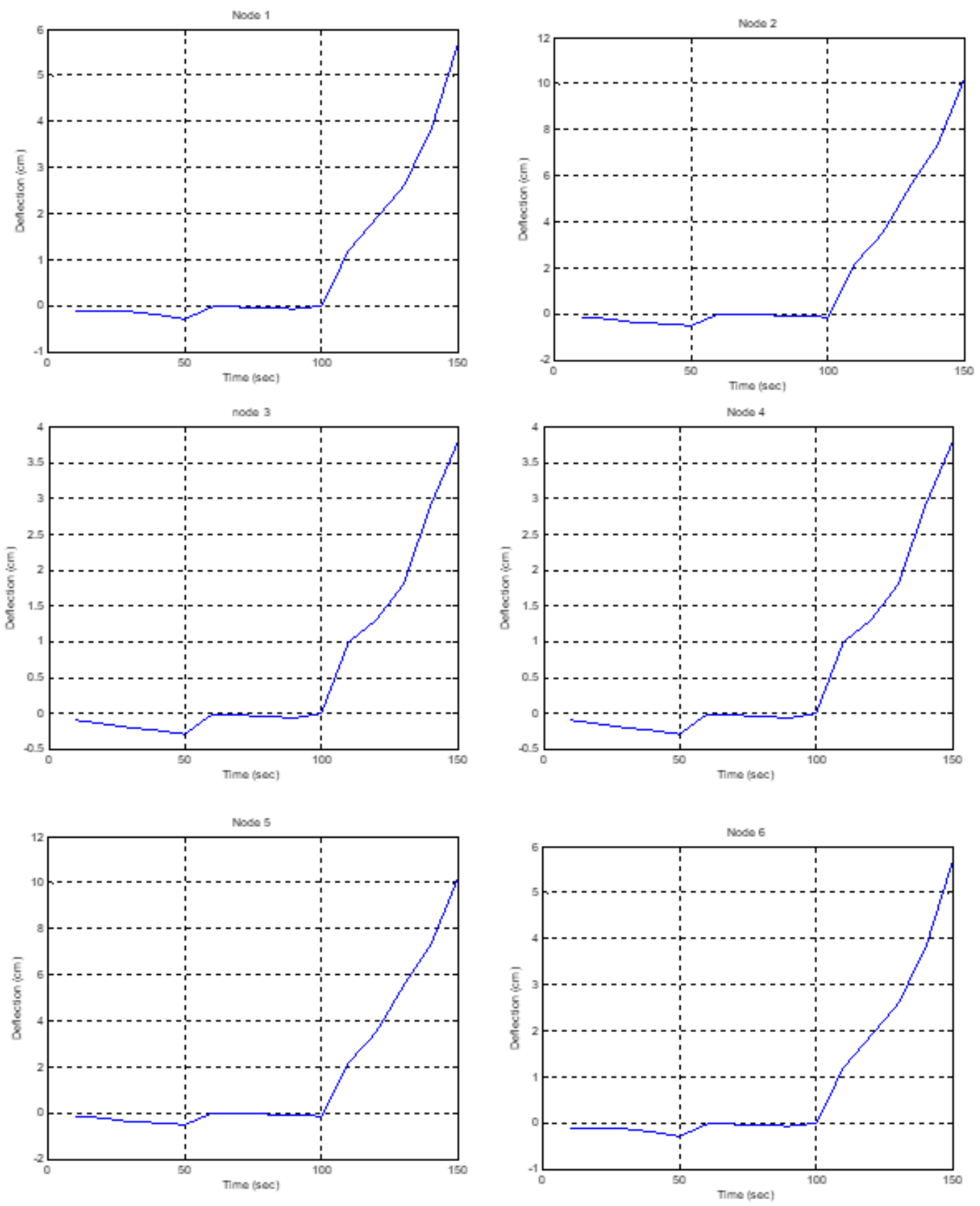

Figure 7.25: Tracking points along the experimental tests 
Fig. 7.26 shows the maximum wing deflection for five value of wind speed, when the AOA greater than zero, a slight increase in deflection is visible when the speed increased, and significant maximum deflection records at the $31 \mathrm{~m} / \mathrm{s}$.

The deflection decreased when the speed increased at $\mathrm{AOA}=-20^{0}$, and $0^{0}$, as shown in Fig. 7.27, and 7.28, while the positive deflection value at $\mathrm{AOA}=+20^{0}$ is increased when the speed increased as shown in Fig. 7.29.

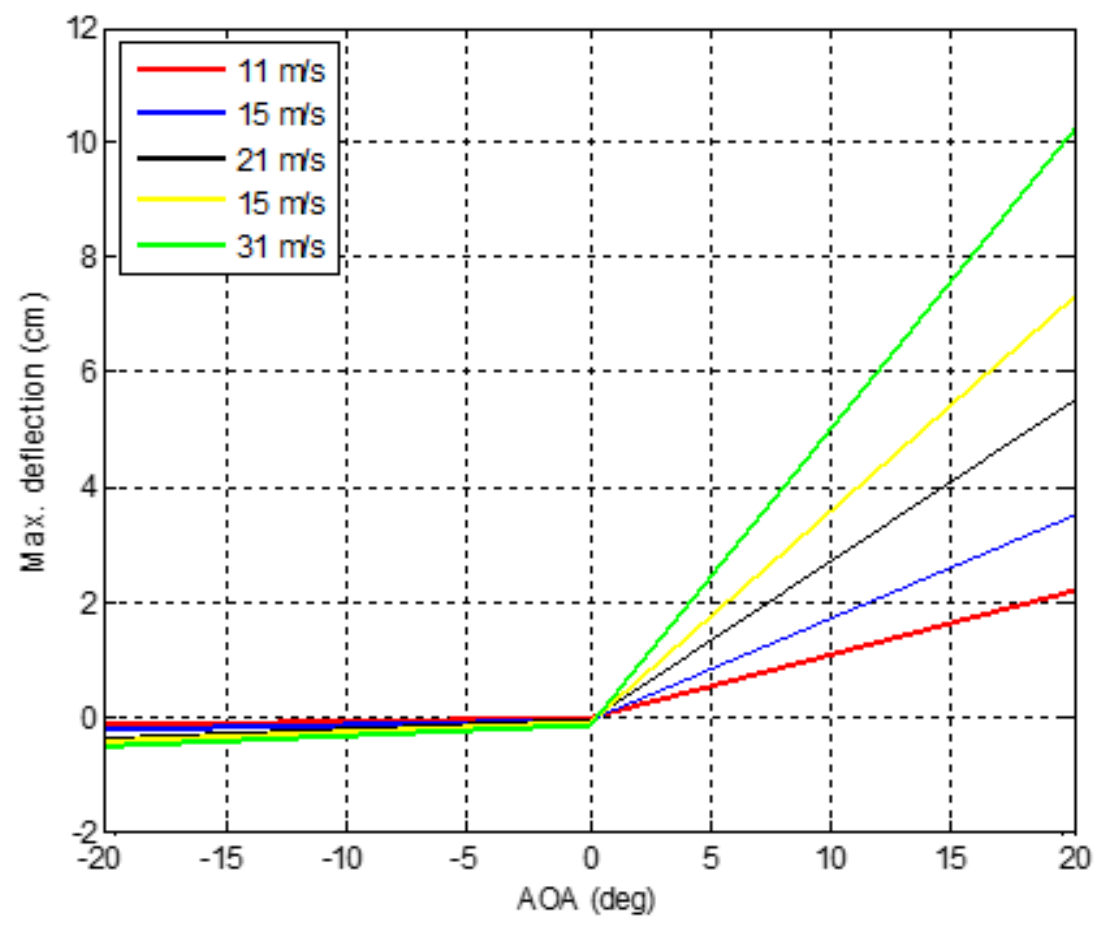

Figure 7.26: Maximum deflection vs. AOA 


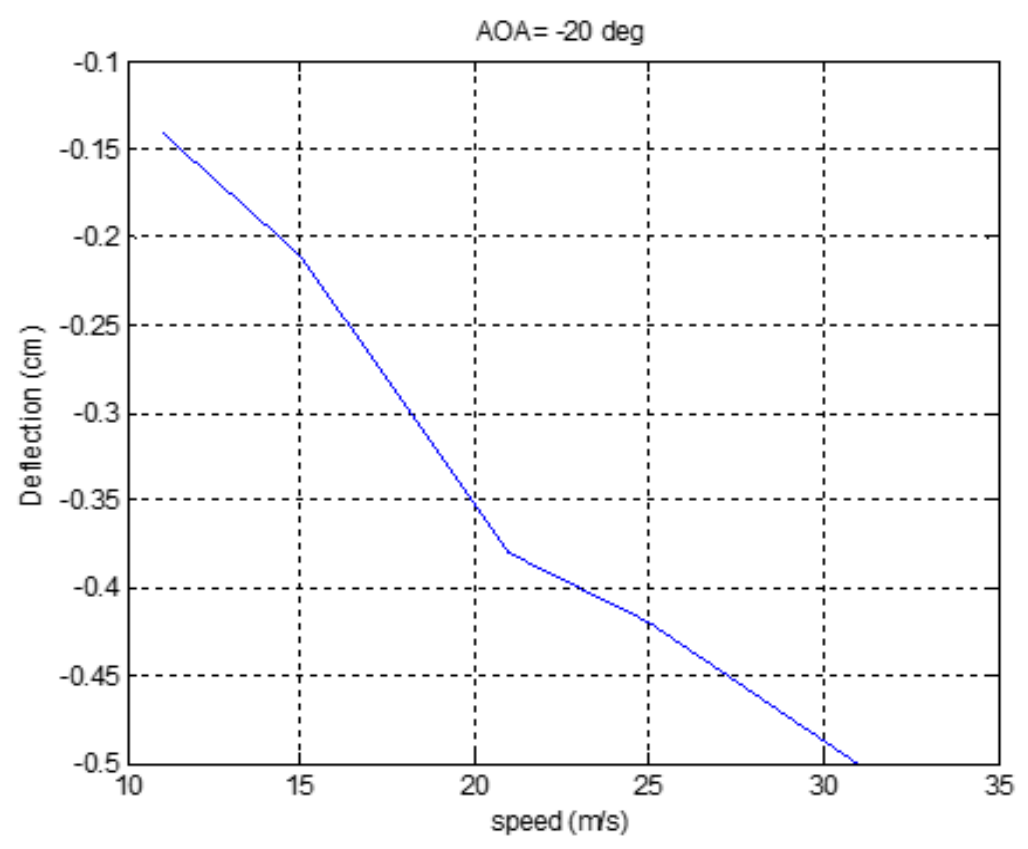

Figure 7.27: Maximum deflection vs. speed at $\mathrm{AOA}=-20 \mathrm{deg}$.

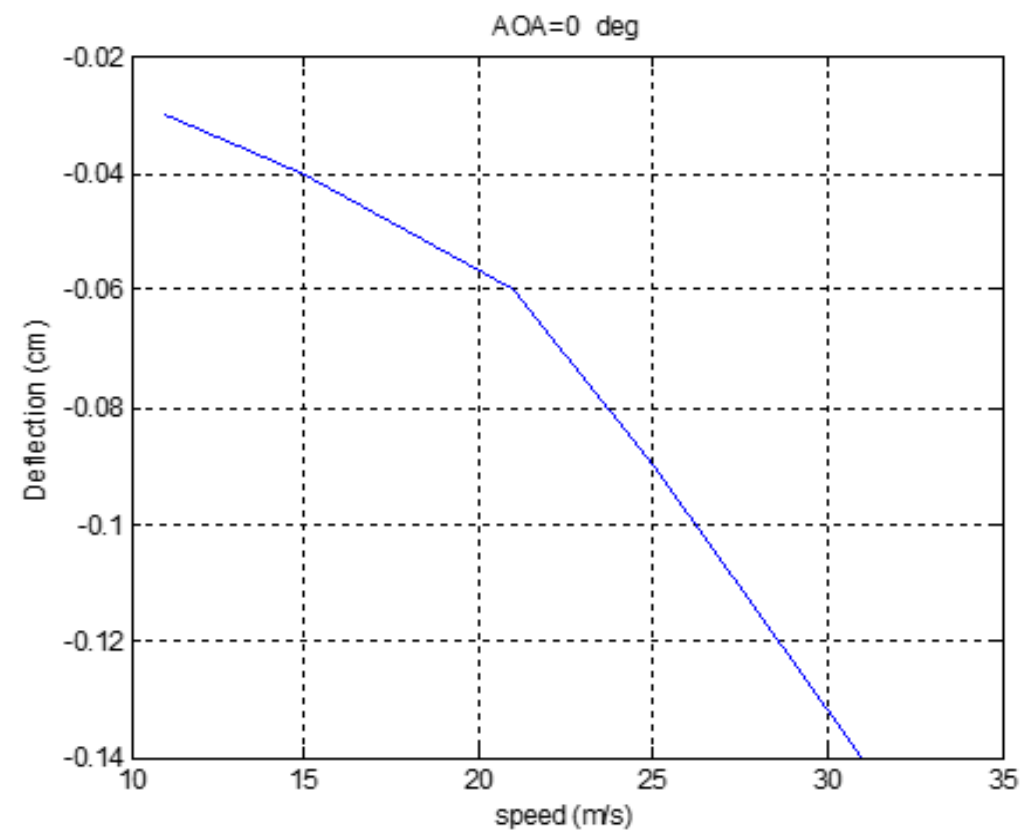

Figure 7.28: Maximum deflection vs. speed at $\mathrm{AOA}=0 \mathrm{deg}$. 


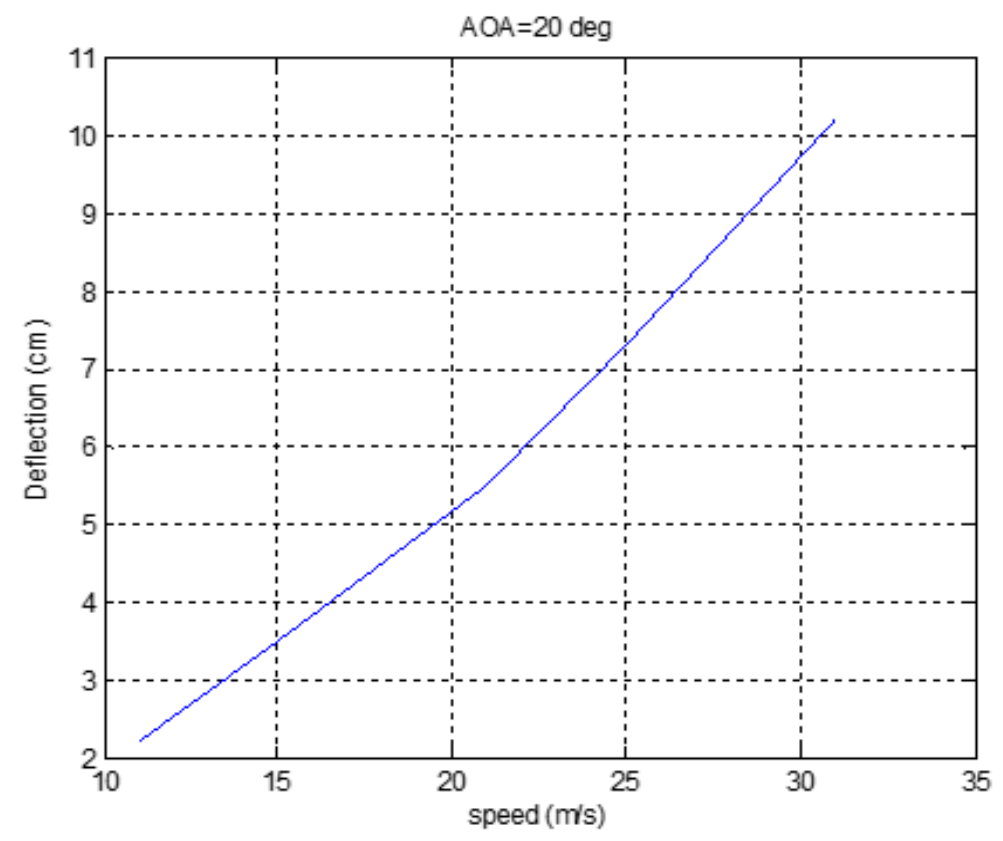

Figure 7.29: Maximum deflection vs. speed at $A O A=20$ deg.

\subsubsection{Classifying Wing Shapes with Fuzzy Logic}

The shapes of a flexible wing are determined by the values of the features (nodes). This work presents six nodes that can yield results, and the deflection values of these nodes for both sides are presented in Table 7.7. Thus, the input variables for fuzzy logic are six nodes, and the output variables are three outputs, as shown in Fig. 7.30. The fuzzy sets of the input variables are defined as negative large, negative middle, negative small, small, middle and large(NL NM NS S, M, L), and the output variables are defined as small, zero and large $(\mathrm{S}, \mathrm{Z}, \mathrm{L})$ for simplicity. The rules of fuzzy logic are shown in Tables 7.8, and 7.9 and the membership function used is triangular. The values of nodes $1,3,4$, and 6 are approximately the same, and according to Table7.7 nodes 1 and node 5 have the same values. Nodes 1 and node 2 were chosen as input membership functions for the fuzzy logic, as shown in Fig.7.31 and 7.32. Fig 7.33, 7.34 and 7.35 illustrate the output variables speed, AOA and roll angle membership 
functions.

Table 7.7: Nodes deflection over the both sides.

\begin{tabular}{|l|l|l|l|l|l|l|l|}
\hline \multicolumn{3}{|c|}{ Classical Input } & \multicolumn{7}{|c|}{ Deflection in cm } \\
\hline & Node No. & NS & NM & NL & S & M & L \\
\hline \multirow{3}{*}{ Left side of wing } & 1 & -0.02 & -0.1 & -0.3 & 0.8 & 3.2 & 5.7 \\
\cline { 2 - 8 } & 2 & -0.035 & -0.14 & -0.5 & 1.05 & 4.3 & 10.2 \\
\cline { 2 - 8 } & 3 & -0.018 & -0.1 & -0.3 & 0.7 & 2.4 & 3.8 \\
\hline \multirow{4}{*}{ Right side of wing } & 4 & -0.018 & -0.1 & -0.3 & 0.7 & 2.4 & 3.8 \\
\cline { 2 - 8 } & 5 & -0.035 & -0.14 & -0.5 & 1.05 & 4.3 & 10.2 \\
\cline { 2 - 8 } & 6 & -0.02 & -0.1 & -0.3 & 0.8 & 3.2 & 5.7 \\
\hline
\end{tabular}

Fuzzy controller

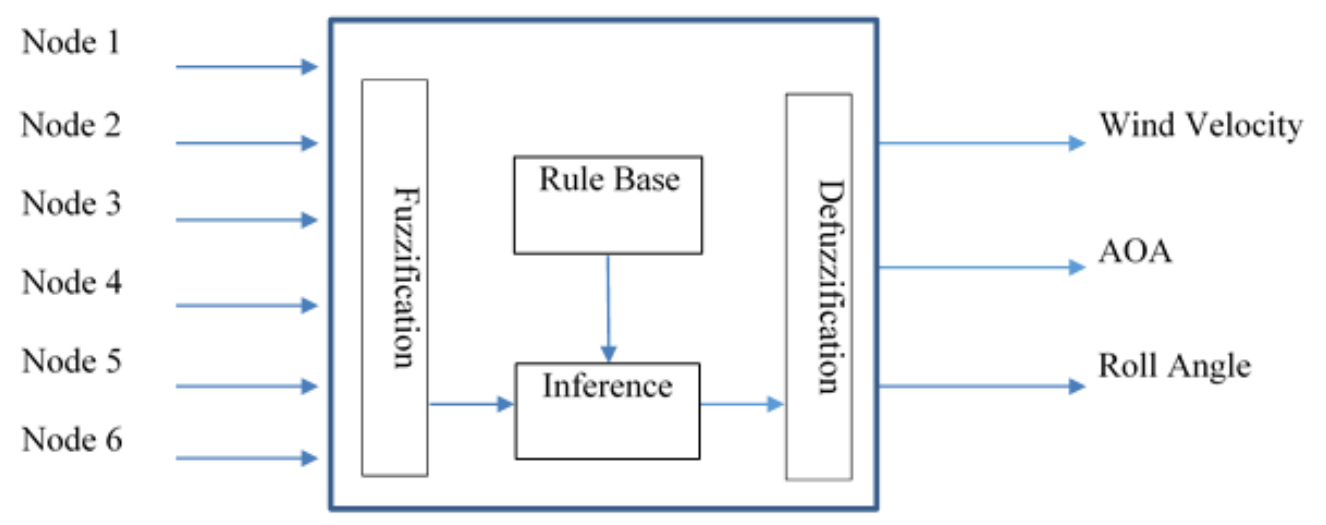

Figure 7.30: Block diagram of Fuzzy logic 
Table 7.8: Fuzzy rules for the flexible wing at a positive deflection

\begin{tabular}{|l|l|l|l|l|}
\hline \multirow{2}{*}{ V, AOA, R } & \multicolumn{3}{|c|}{ Node 1,2,3 } \\
\cline { 3 - 5 } \multicolumn{2}{|c|}{} & S & M & L \\
\hline \multirow{4}{*}{ Node 4,5,6 } & S & S, M, Z & M, P, N & L, P, N \\
\cline { 2 - 5 } & M & M, P, P & M, M, Z & M, P, N \\
\cline { 2 - 5 } & L & L, P, P & M, P, P & L, L, Z \\
\hline
\end{tabular}

Table 7.9: Fuzzy rules for the flexible wing at a negative deflection.

\begin{tabular}{|c|l|l|l|l|}
\hline \multicolumn{2}{|c|}{ V, AOA, R } & \multicolumn{3}{|c|}{ Node $1,2,3$} \\
\cline { 3 - 5 } \multicolumn{2}{|c|}{} & NL & NM & NS \\
\hline \multirow{3}{*}{ Node 4,5,6 } & NL & L, Z, Z & M, N, N & L, N, N \\
\cline { 2 - 5 } & NM & M, N, P & M, M, Z & M, Z, N \\
\cline { 2 - 5 } & NS & L, N, P & M, Z, P & S, N, Z \\
\hline
\end{tabular}

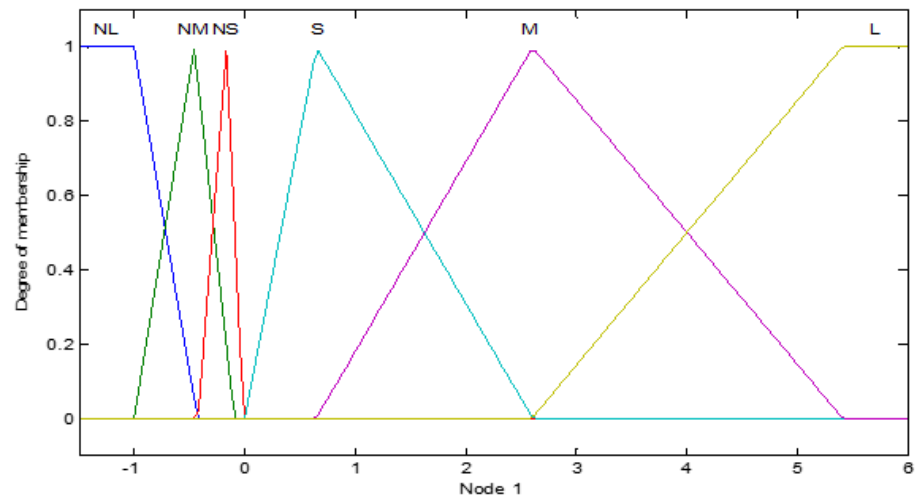

Figure 7.31: Membership function of the input node 1 


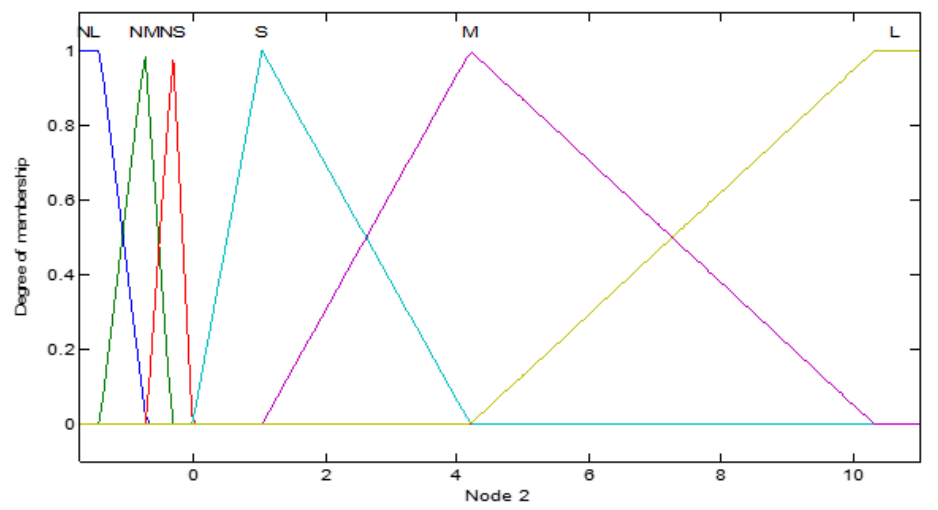

Figure 7.32: Membership function of the input node 2

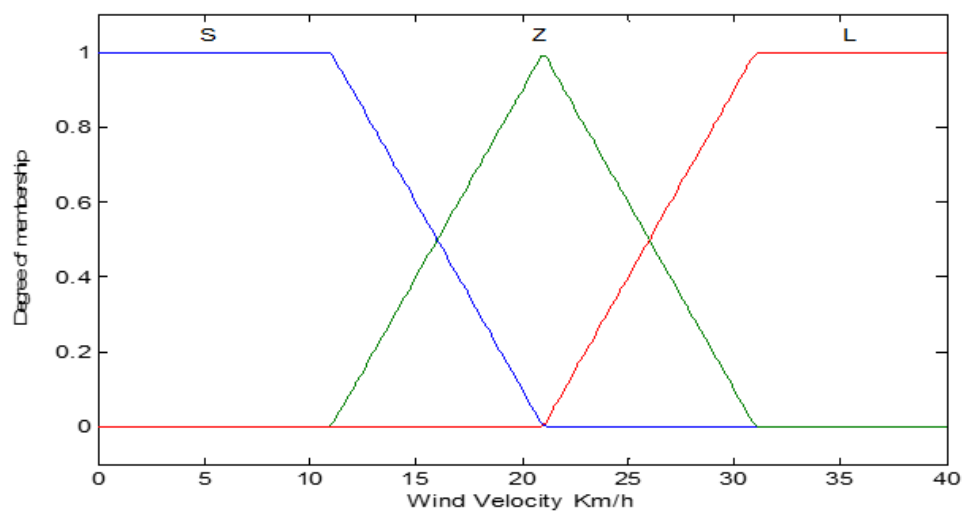

Figure 7.33: Membership function of the output wind speed

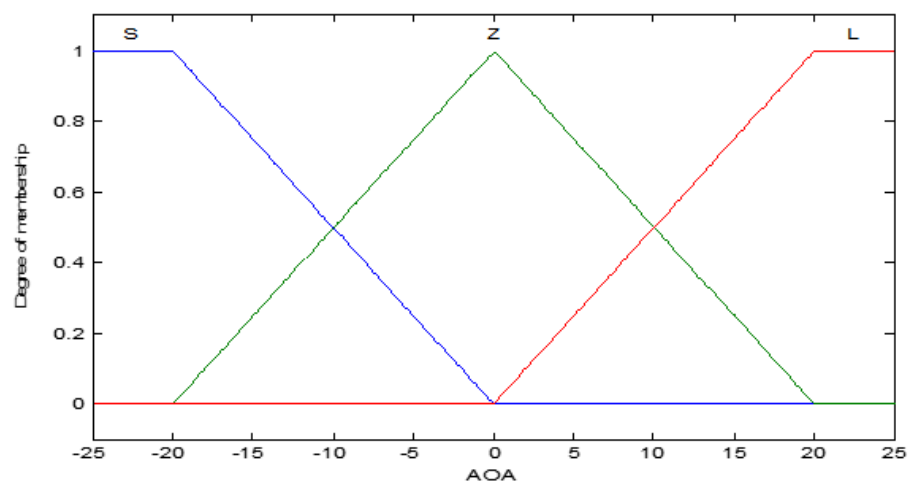

Figure 7.34: Membership function of the output AOA 


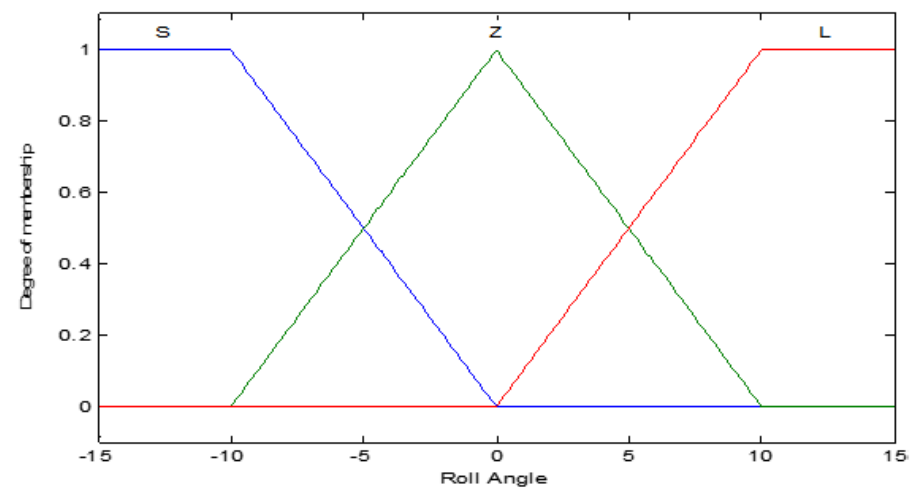

Figure 7.35: Membership function of the output roll angle

\subsubsection{Test and Validation}

The proposed DDVS method for experimental testing is evaluated by different sensors using AUKF, and the experiments are conducted using a flexible wing UAV in a wind tunnel, as shown in Fig. 7.36. An Arduino board with an accelerometer sensors was designed and built for the wing, as shown in Fig. 7.37. The goal of the experiments is to evaluate the accuracy of the attitude angles (pitch and roll) estimates and to test the proposed DDVS. Table 7.10 shows the experimental results for both the pitch and roll angles. It is noticed that the results coming from the proposed method is more accurate than the accelerometer results.

The pitch error and roll error that determined from the accelerometer, and DDVS, and the attitude error estimated by AUKF are shown in Fig. 7.38 and 7.39 respectively. It was found that the error associated with the proposed estimated 2-DOF attitude acceptable in comparison the attitude error reported by the accelerometer. While the error associated with AUKF algorithm is approached zero compared to the attitude error reported by both the accelerometer and DDVS. This strongly suggests that the AUKF estimates of roll and pitch are significantly more accurate than those 


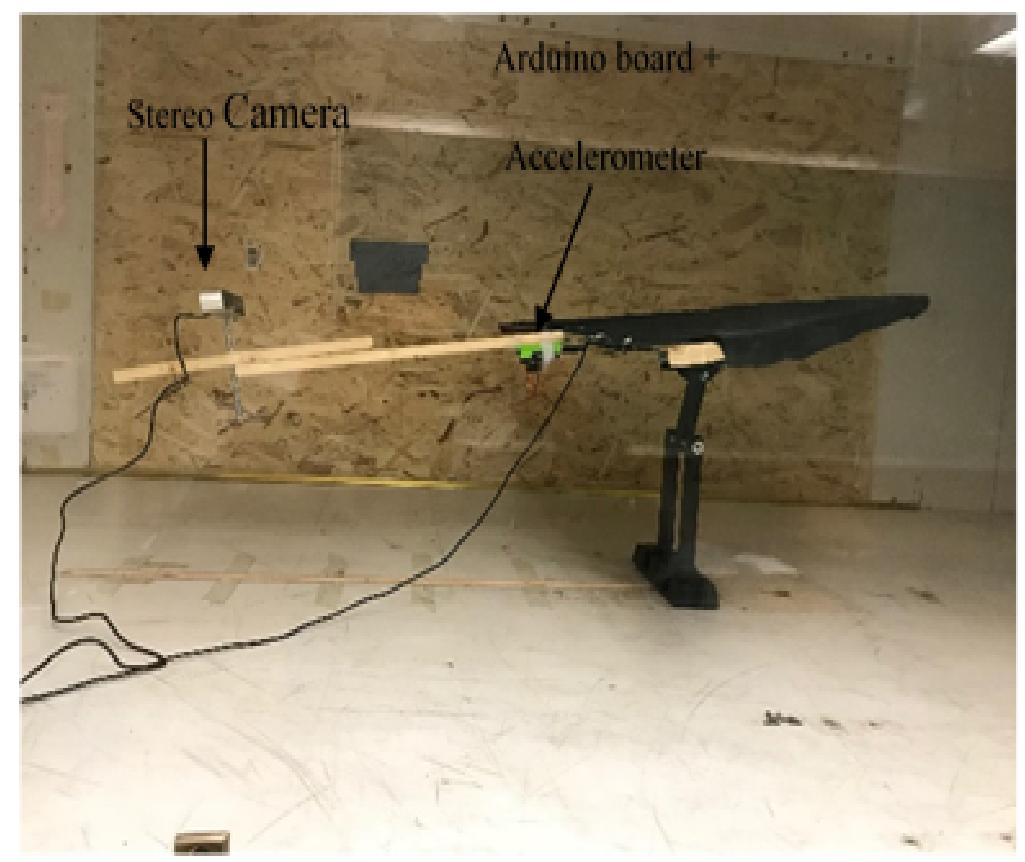

Figure 7.36: Two sensor are fixed in flexible wing

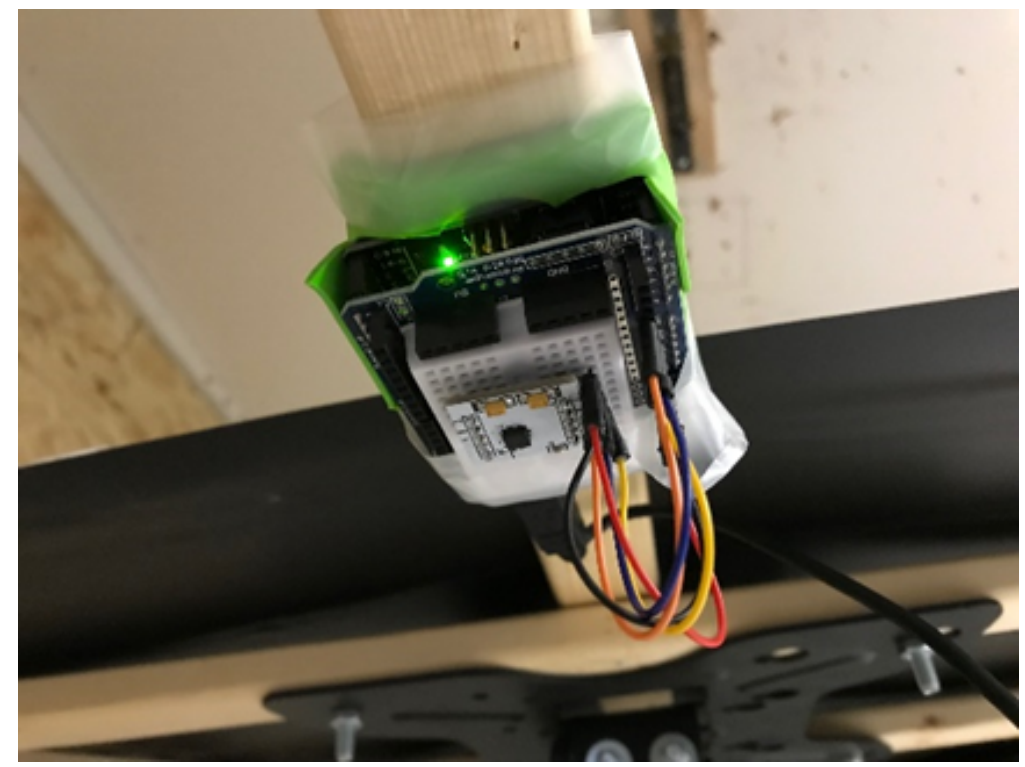

Figure 7.37: Accelerometer sensor with Arduino board

from the accelerometer and the DDVS. 
Table 7.10: Experimental results for attitude angles

\begin{tabular}{|l|l|l|l|}
\hline & Accelerometer & DDVS & True \\
\hline & 0 & 0 & 0 \\
\cline { 2 - 4 } & 8.21 & 9.71 & 10.0 \\
\cline { 2 - 4 } & 13.2 & 14.1 & 15.0 \\
\cline { 2 - 4 } Pitch angle (degree) & 17.8 & 20.3 & 20.0 \\
\cline { 2 - 4 } & 28.1 & 29.3 & 30.0 \\
\cline { 2 - 4 } & -8.45 & -9.72 & -10.0 \\
\cline { 2 - 4 } Roll angle (degree) & -13.5 & -14.3 & -15.0 \\
\cline { 2 - 4 } & -17.9 & -19.7 & -20.0 \\
\cline { 2 - 4 } & -28.2 & -29.4 & -30.0 \\
\hline & 0 & 0 & 0 \\
\cline { 2 - 4 } & 8.82 & 9.51 & 10.0 \\
\cline { 2 - 4 } & -7.4 & 14.3 & 15.0 \\
\cline { 2 - 4 } & -13.1 & -9.45 & -10.0 \\
\hline
\end{tabular}




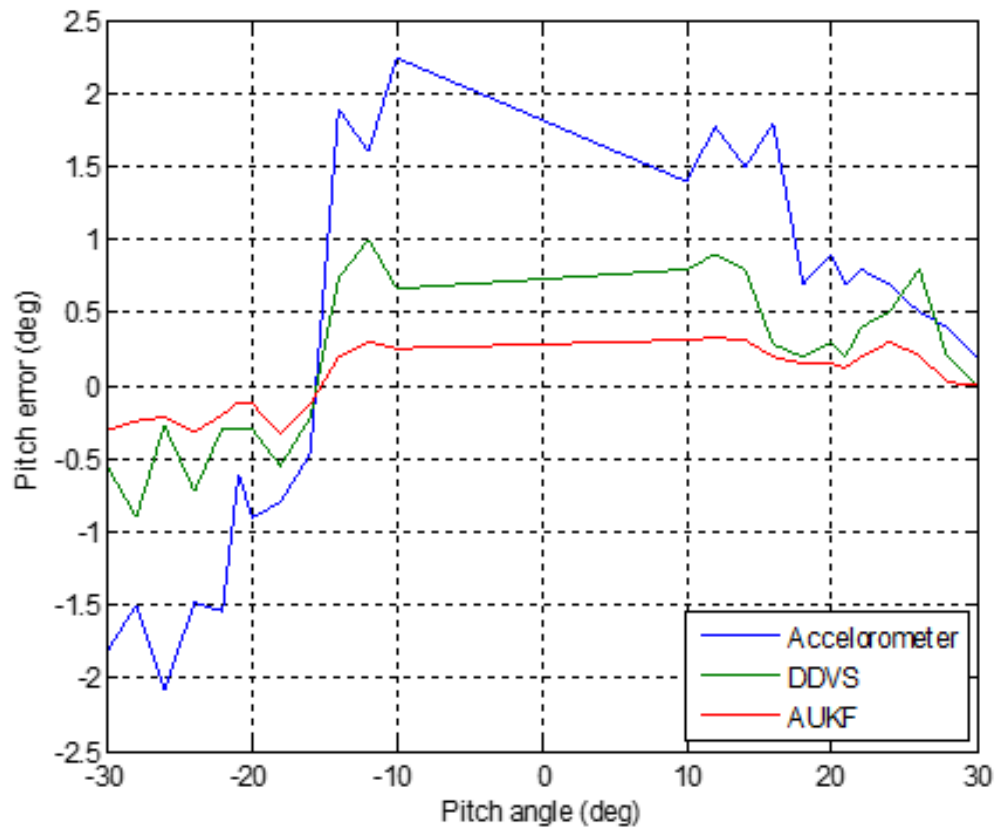

Figure 7.38: Pitch error for the proposed DDVS, accelerometer and AUKF

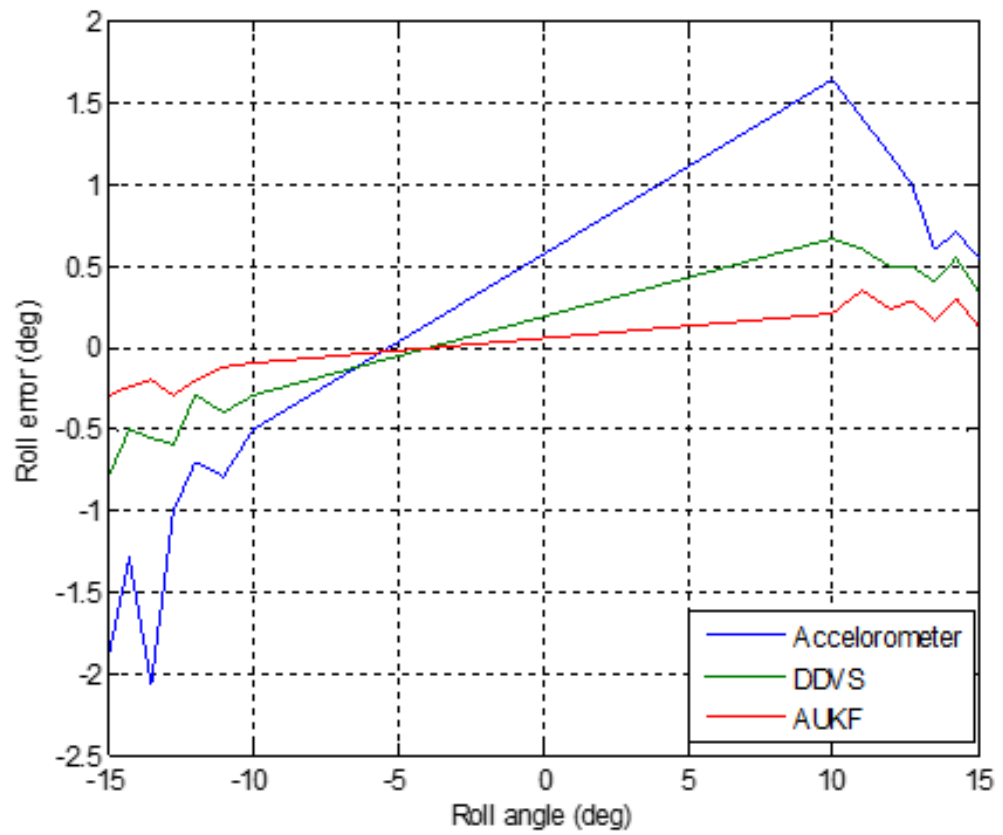

Figure 7.39: Roll error for the proposed DDVS, accelerometer and AUKF 


\subsection{Sensor Fusion}

The sensor fusion algorithm is a technique to integrate different attitude measurements to provide a reliable representation of attitude measurement. The measurement data from sensors such as accelerometer, Gyro and DDVS proposal are combined using two filters: a regular UKF and a new AUKF. The data for both pitch and roll angles comes instantaneously from the two sensors at precisely the same time.

The dynamics equations for the UAV is driven based on the relationship between the UAV body rate (pitch, roll, and yaw rates) and the four parameters of the quaternion method described in Equation (6.3). Thus, the dynamic system can be expressed as [86]:

$$
\begin{aligned}
& \dot{x}=A x+w \\
& z=h(x)+v
\end{aligned}
$$

where $\mathrm{x}=\left[q_{0}, q_{1}, q_{2}, q_{3}\right]$ the state vector, $\mathrm{z}=\left[a_{x}, a_{y}, a_{z}\right]$ measurements vector where $a_{x}, a_{y}$ and $a_{z}$ are the compensated accelerations and $\mathrm{w}$ and $\mathrm{v}$ are processed noise and measurement noise, and are assumed to be uncorrelated Gaussian distributed white noise. The $\mathrm{A}$ and $\mathrm{h}(\mathrm{x})$ are defined as [86] [94]:

$$
A=\frac{1}{2}\left[\begin{array}{cccc}
0 & -p & -q & -r \\
p & 0 & r & -q \\
q & -r & 0 & p \\
r & q & -p & 0
\end{array}\right]
$$




$$
h(x)\left[\begin{array}{c}
2\left(q_{1} q_{3}-q_{0} q_{2}\right) \\
2\left(q_{2} q_{3}-q_{0} q_{1}\right) \\
q_{0}^{2}-q_{1}^{2}-q_{2}^{2}+q_{3}^{2}
\end{array}\right]
$$

The measurements of pitch angle and roll anglesare determined based on (6.7):

$$
\left[\begin{array}{l}
\theta \\
\phi
\end{array}\right]\left[\begin{array}{c}
\operatorname{atan} \frac{a_{y}}{\sqrt{a_{x}^{2}+a_{z}^{2}}} \\
\operatorname{atan} \frac{-a_{x}}{a_{z}}
\end{array}\right]
$$

For the simulation and experimental results, the noise covariance associated to the quaternion components are:

$Q=1 \times 10^{-6} . I_{4 \times 4}$ According to the standard deviation obtained from the measurement of the accelerometer, the measurement noise of the accelerometer $\mathrm{R}$ is

$$
R=\operatorname{diag}[0.0015, \quad 0.0015, \quad 0.0015]
$$

The fuzzy rules for AUKF are shown in Table 7.11, and the membership function for the input and output variables are shown in Fig. 7.40, 7.41 and 7.42 respectively.

Table 7.11: The Fuzzy Rule

\begin{tabular}{|l|l|l|l|}
\hline$\xi / \mu$ & $\mathrm{Z}$ & $\mathrm{S}$ & $\mathrm{L}$ \\
\hline $\mathrm{Z}$ & $\mathrm{S}$ & $\mathrm{Z}$ & $\mathrm{Z}$ \\
\hline $\mathrm{S}$ & $\mathrm{S}$ & $\mathrm{M}$ & $\mathrm{S}$ \\
\hline $\mathrm{L}$ & $\mathrm{L}$ & $\mathrm{L}$ & $\mathrm{M}$ \\
\hline
\end{tabular}

Z: Zero; S: Small; M:Medium; L: Large 


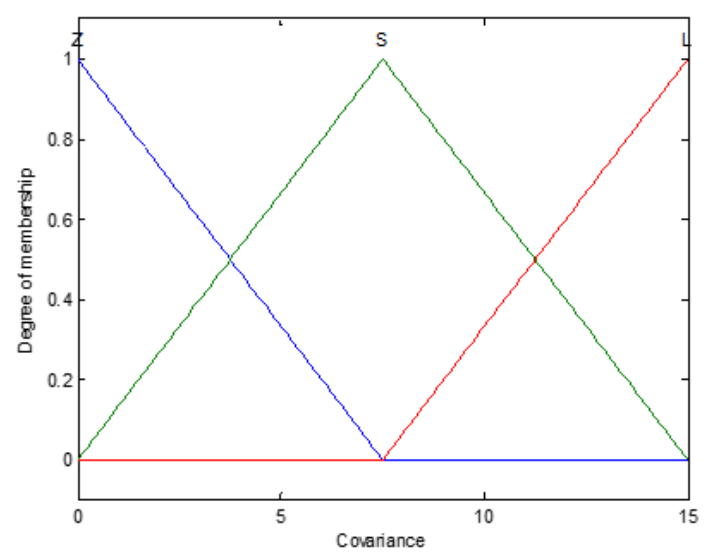

Figure 7.40: Covariance membership function of input variable

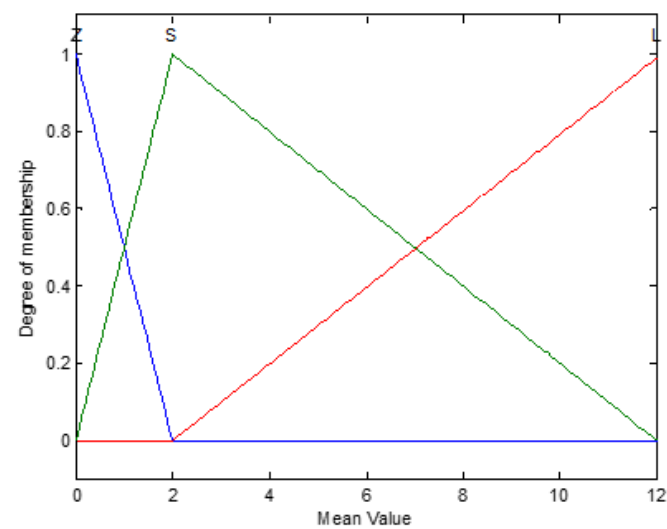

Figure 7.41: Mean value membership function of input variable

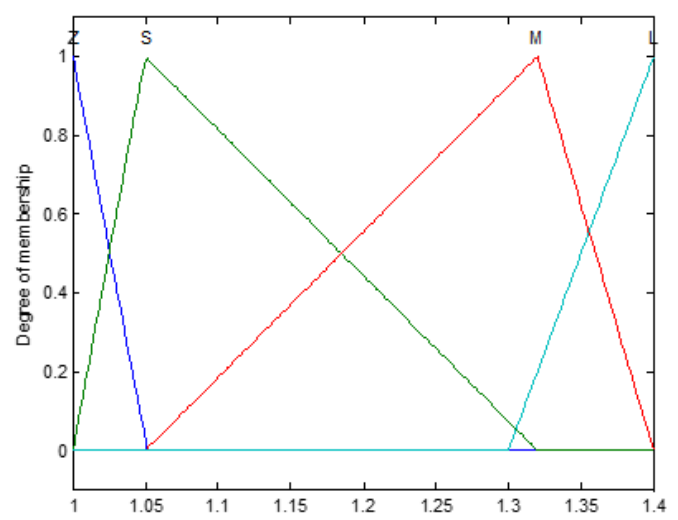

Figure 7.42: Membership function of output variable 
Fig. 7.43 shows the estimation pitch angles with AUKF and UKF, and compares them with the real attitude angles when the pitch angle varies from - 15 to 30 degrees for 40 sec. The pitch angle estimate by AUKF was found to be more accurate than the UKF estimate. Fig. 7.44 shows the roll angle result for both the AUKF and UKF filters when the angle changes from -15 to 15 degrees, and the AUKF It noticed that the noise in the roll angle was reduced with AUKF and the results is better compared to the result estimation by UKF.

The error in pitch and roll angles for the flexible wing UAV are shown in Fig. 7.45 and 7.46. As can be seen, the AUKF estimate errors are smaller than the UKF estimation error for both pitch and roll angles. However, the AUKF employs the fuzzy system to detect and prevent the UKF from divergence by monitoring the residual states. The efficiency of the proposed algorithm to estimate pitch and roll angles and their errors with the AUKF and UKF filters is shown in Fig. 7.45 and 7.46. In both cases, the same data gathered from the accelerometer and DDVS are used in the filter equations. As seen, the AUKF clearly a better performance than the UKF. 


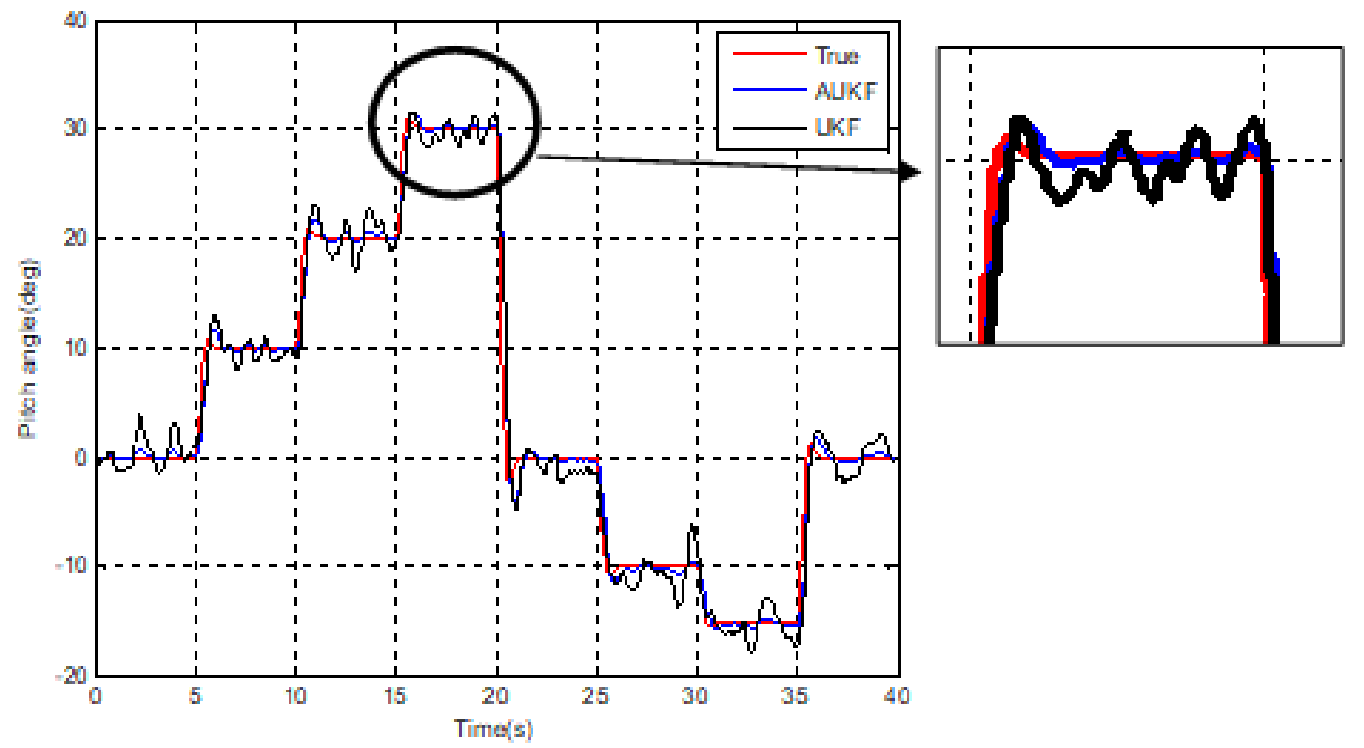

Figure 7.43: Pitch angle estimation obtained from the UKF and the AUKF

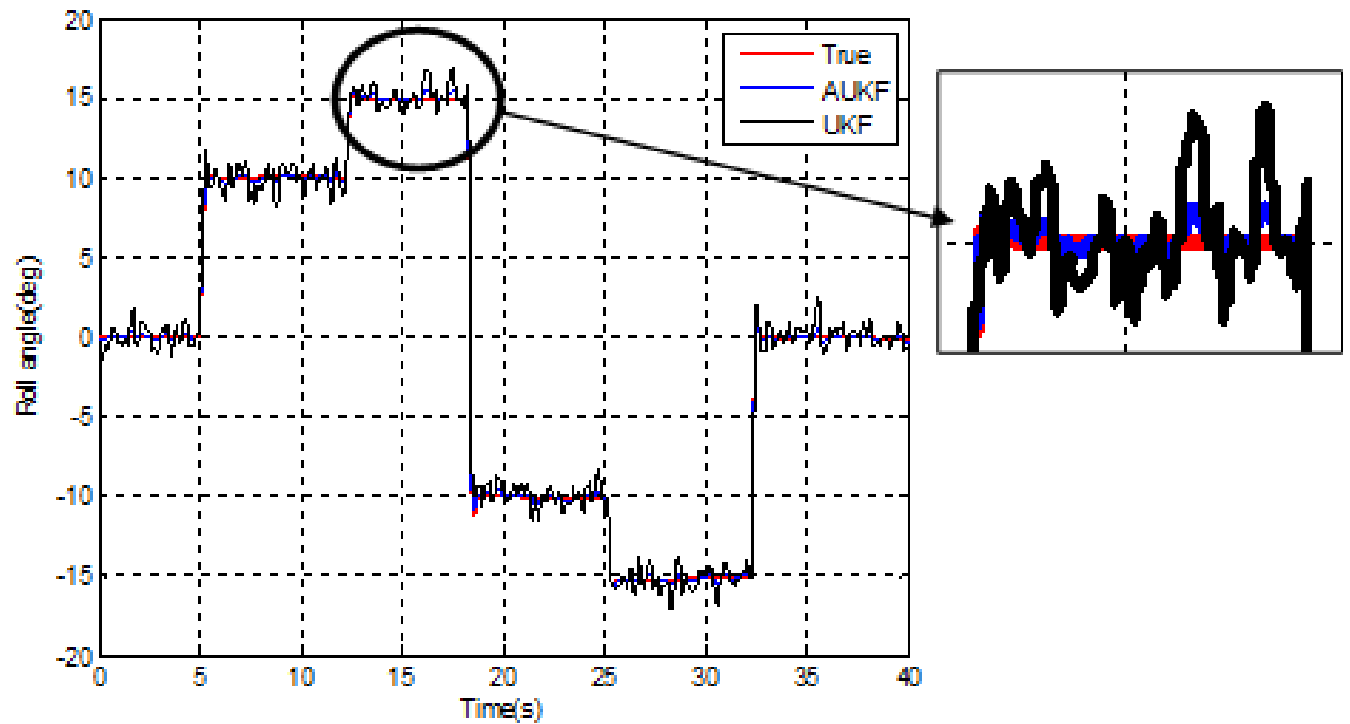

Figure 7.44: Roll angle estimation obtained from the UKF and the AUKF 


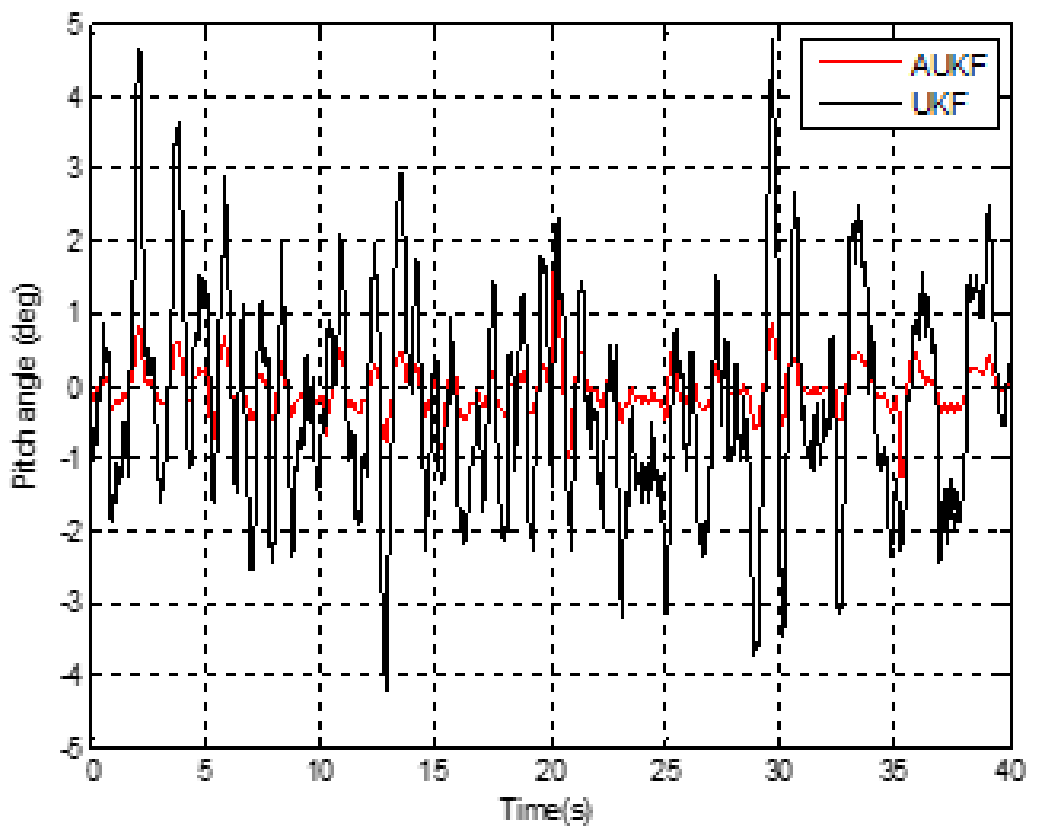

Figure 7.45: The error estimation of pitch angle

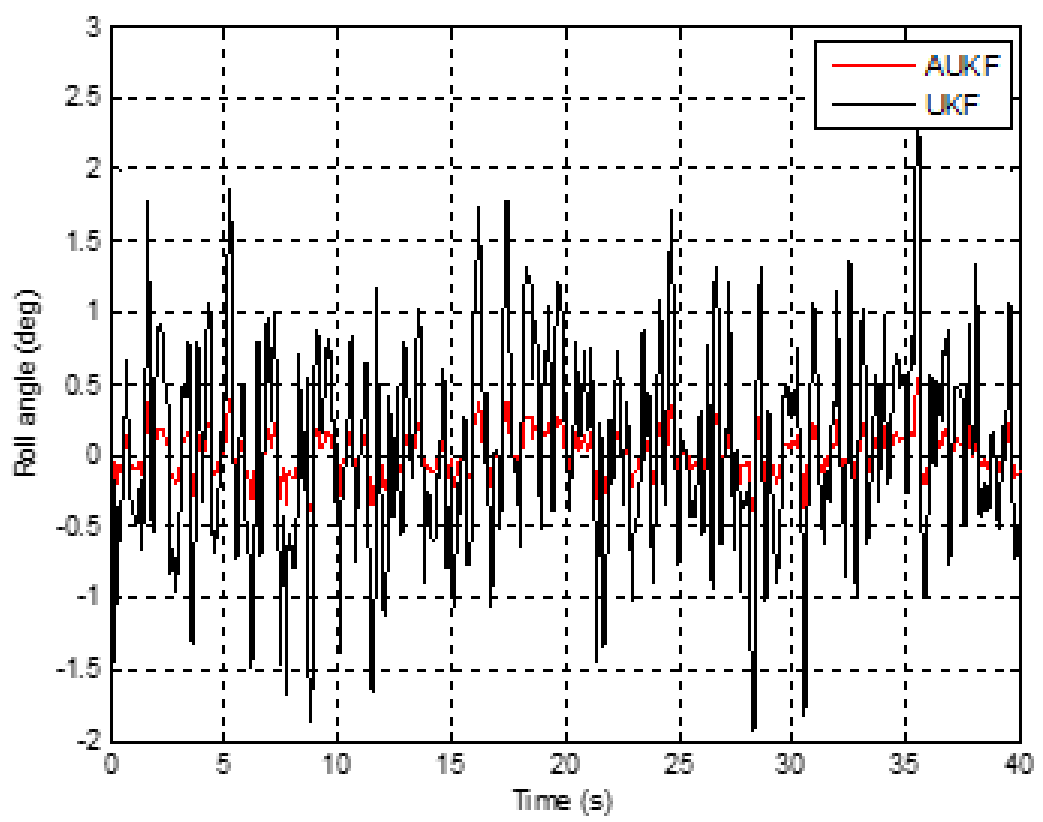

Figure 7.46: The error estimation of roll angle 


\subsection{Autopilot}

\subsubsection{Longitudinal Autopilot Simulation}

For the longitudinal control design, the ANFIS control method is applied and compared with the PID controller. The state-space model used to design these controllers is given as [4].

$$
\left[\begin{array}{c}
\dot{u} \\
\dot{w} \\
\dot{q} \\
\dot{\theta}
\end{array}\right]=\left[\begin{array}{cccc}
-0.179 & 0.675 & 0.121 & -0.350 \\
-1.466 & -2.326 & 8.331 & 0.821 \\
0.172 & -0.281 & -0.555 & 0 \\
0 & 0 & 1 & 0
\end{array}\right]\left[\begin{array}{c}
u \\
w \\
q \\
\theta
\end{array}\right]+\left[\begin{array}{c}
0 \\
0 \\
4.231 \\
0
\end{array}\right] \delta_{\text {long }}
$$

The state-space matrices are determined using aerodynamic stability derivatives calculated in Chapter 4, and the explanation can be found in Appendix A. The controllability matrix is

$$
C_{n t r}=\left[\begin{array}{lllll}
B & A B & A^{2} B & \ldots & A^{n-1} B
\end{array}\right]
$$

The matrix $C_{n t r}$ has full rank so, the system is controllable.

The inference system for longitudinal autopilot is comprised of three sub-systems: pitch angle, altitude and speed, all with six membership functions and 36 fuzzy rules. The input-output data of the PID controller are used as training data at different operating points. The first inputs for the inference system are the pitch, altitude, and speed errors, which are the differences between the desired and real values. The other inputs are the derivative errors for pitch, altitude and speed errors, which are also the differences between the desired and the real values. The input membership 
functions and the output surfaces for pitch angle, altitude and speed are shown in Fig. 7.47, 7.48, 7.49, 7.50, 7.51, 7.52. 7.53, 7.54 and 7.55.

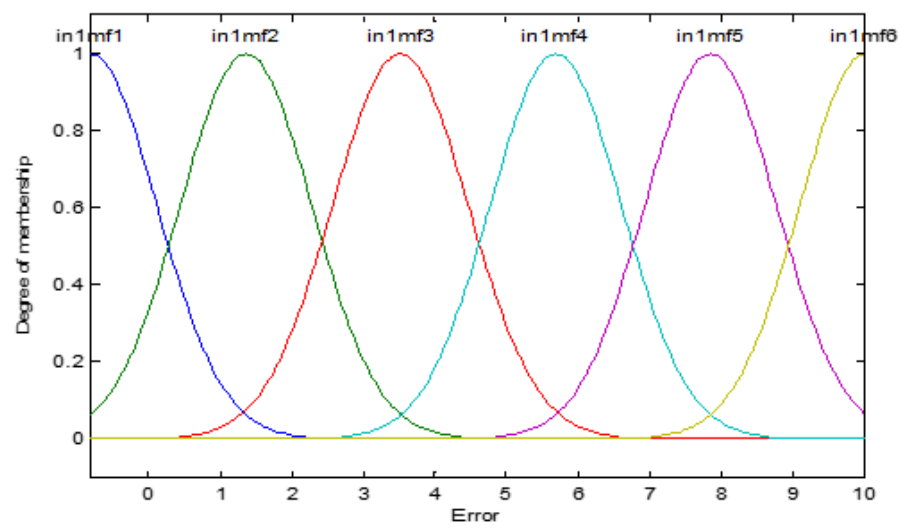

Figure 7.47: Input variable "error" for pitch angle

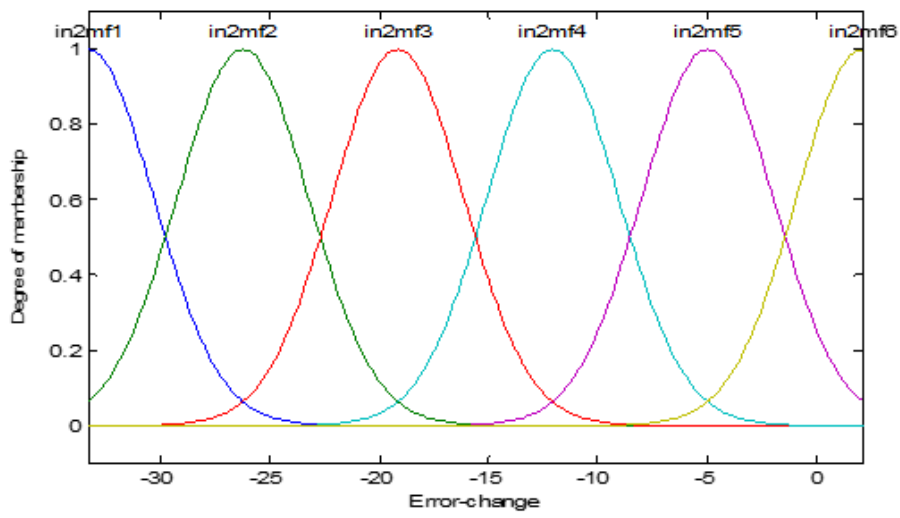

Figure 7.48: Input variable "error change" for pitch angle 


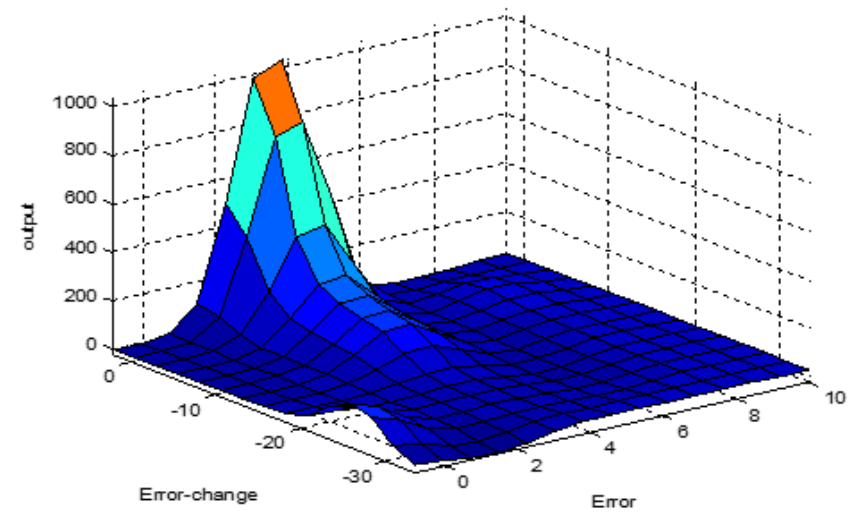

Figure 7.49: Output surface of fuzzy modules for pitch angle

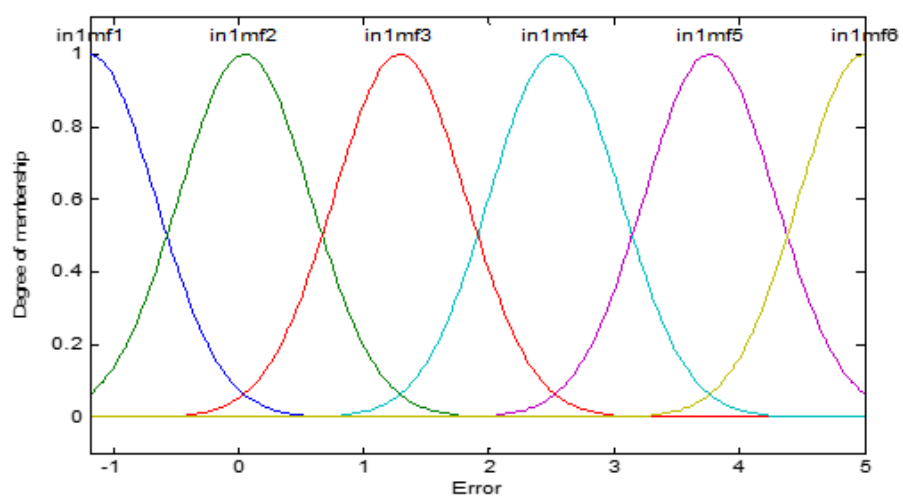

Figure 7.50: Input variable "error" for altitude control

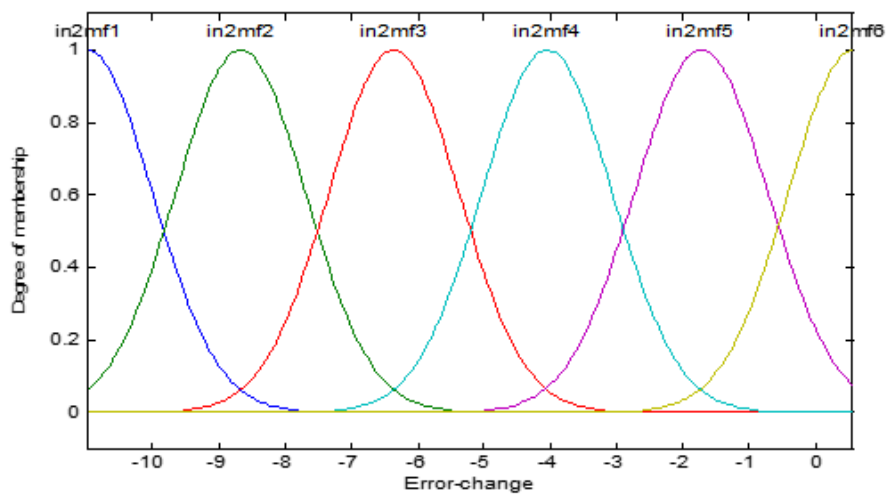

Figure 7.51: Input variable "error change" for altitude control 


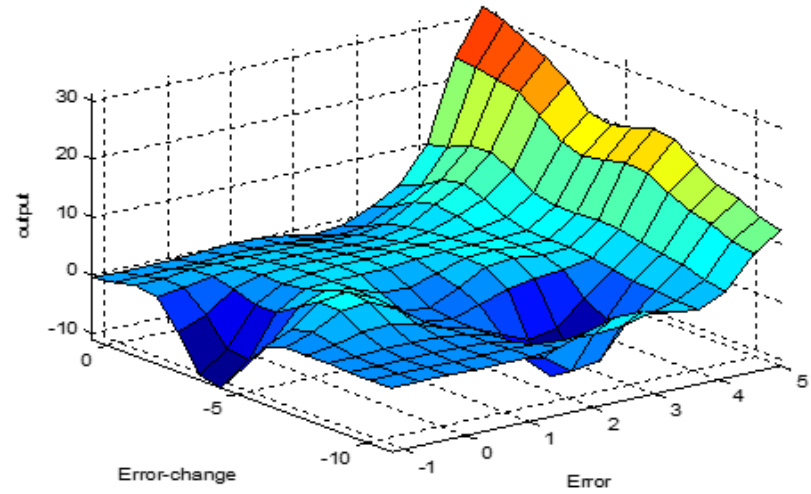

Figure 7.52: Output surface of fuzzy modules for altitude control

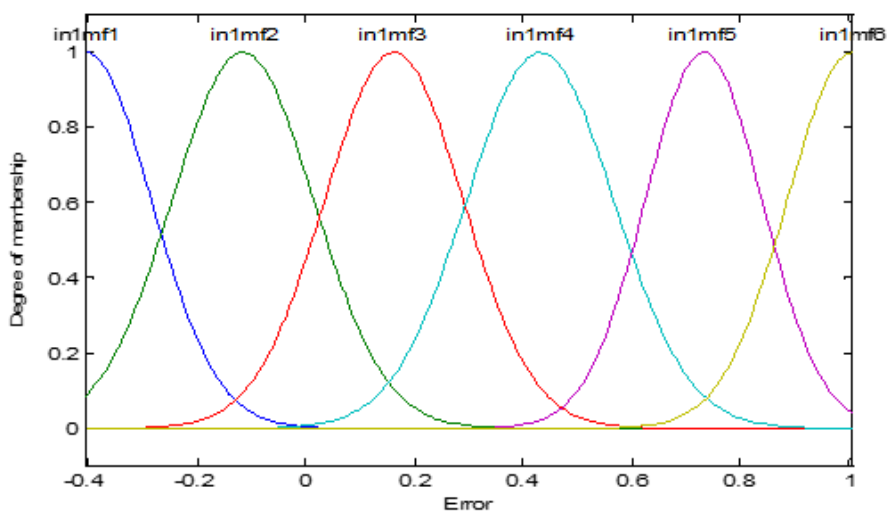

Figure 7.53: Input variable "error" for speed control

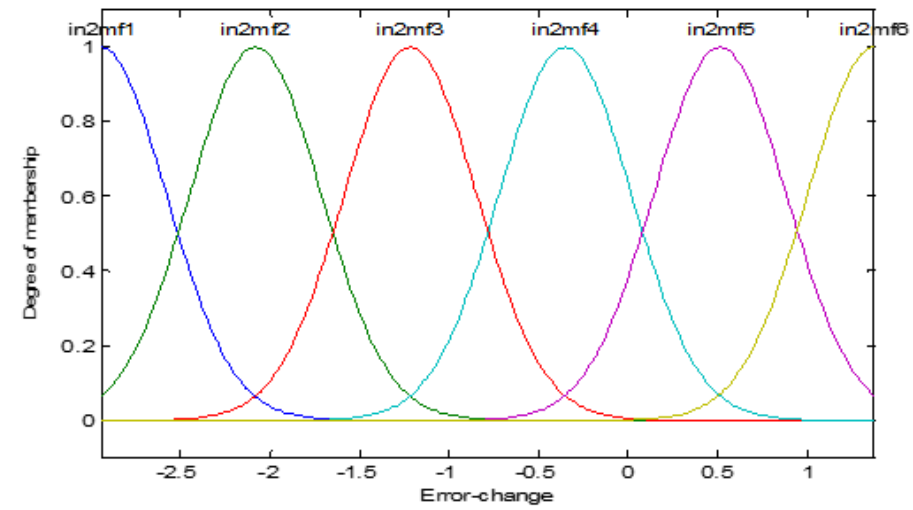

Figure 7.54: Input variable "error change" for speed control 


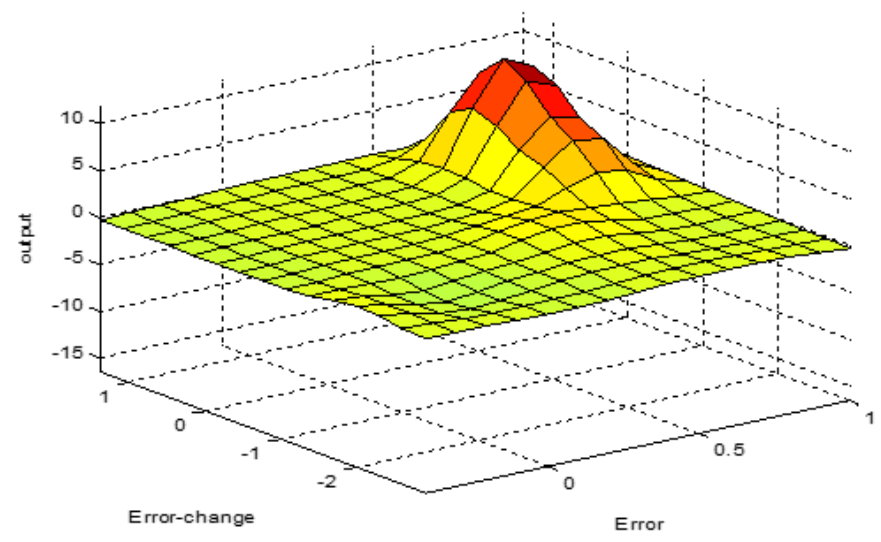

Figure 7.55: Output surface of fuzzy modules for speed control

\subsubsection{Time Domain Analysis}

The longitudinal autopilot performance for hang glider is simulated in three stages. First, the PID and ANFIS controllers are simulated in the inner pitch loop by moving a step input control bar $\left(\delta_{l o n}\right)$ to the desired pitch angle when the PID gains are $\left(k_{p}=1.2 ; k_{i}=1\right.$ and $\left.k_{d}=0.2\right)$ as shown in Fig. 7.56. Second, the altitude response for two controllers is simulated by moving a step input control bar $\left(\delta_{\text {lon }}\right)$ to the desired altitude when the PID gains are $\left(k_{p}=22 ; k_{i}=11\right.$ and $\left.k_{d}=10\right)$, as shown in Fig. 7.57. Finally, the velocity response for two controllers is shown in Fig. 7.58 when the PID gains are $\left(k_{p}=34 ; k_{i}=24\right.$ and $\left.k_{d}=8\right)$. The longitudinal simulations in Tables 7.12, and 7.13 show the performance of the ANFIS and PID to control the altitude, velocity, and pitch angle of the UAV in response to the step inputs. These tables indicate that the rise time, settling time, overshoot and peak time of the designed controller (ANFIS) are better than those with PID. 
Table 7.12: Time domain analysis of pitch angle

\begin{tabular}{|l|l|l|}
\hline & Designed controller & Classic controller \\
\hline Rise Time(s) & 8.84 & 9.78 \\
\hline Settling Time (s) & 43.8 & 47.3 \\
\hline Overshoot & 7.36 & 12.7 \\
\hline Peak & 10.8 & 11.3 \\
\hline
\end{tabular}

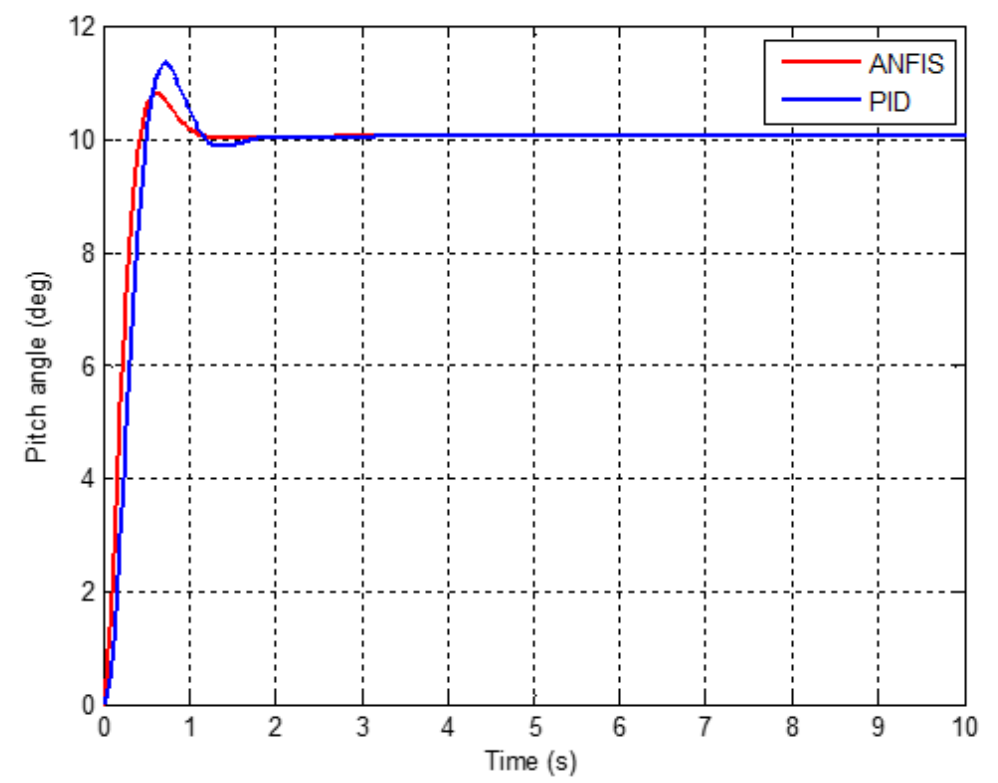

Figure 7.56: Pitch-hold longitudinal autopilot response

Table 7.13: Time domain analysis of speed

\begin{tabular}{|l|l|l|}
\hline & Classic controller & Designed controller \\
\hline Rise Time(s) & 0.25 & 0.23 \\
\hline Settling Time (s) & 6.04 & 3.42 \\
\hline Overshoot & 65.1 & 60.2 \\
\hline Peak & 1.65 & 1.60 \\
\hline
\end{tabular}




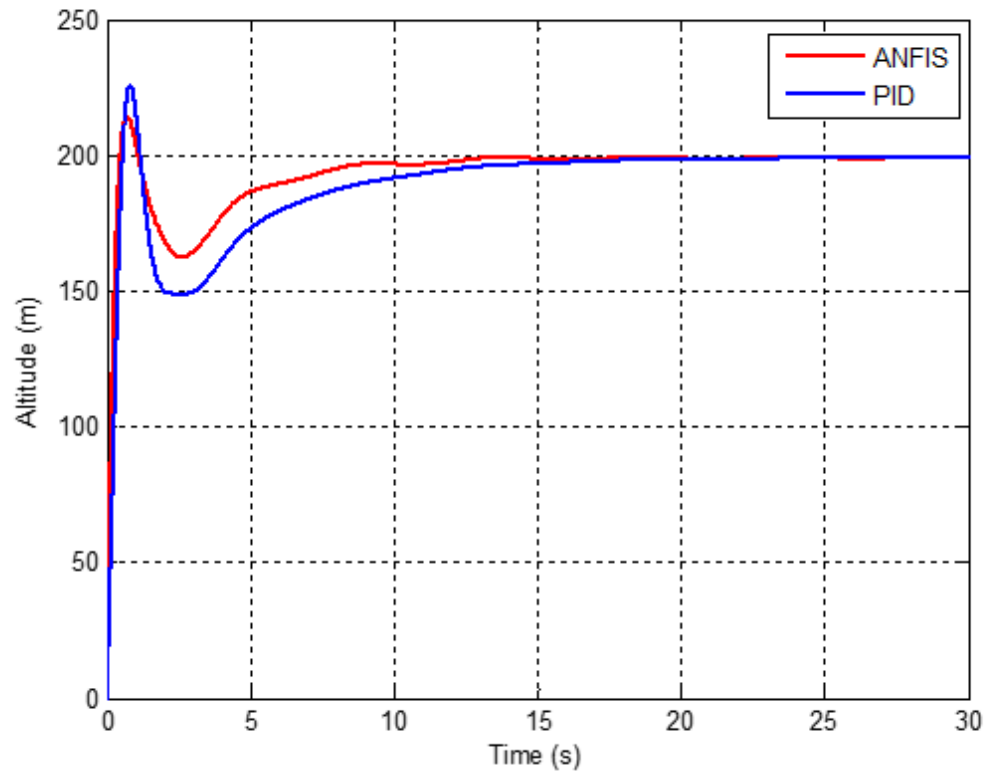

Figure 7.57: Altitude-hold longitudinal autopilot response

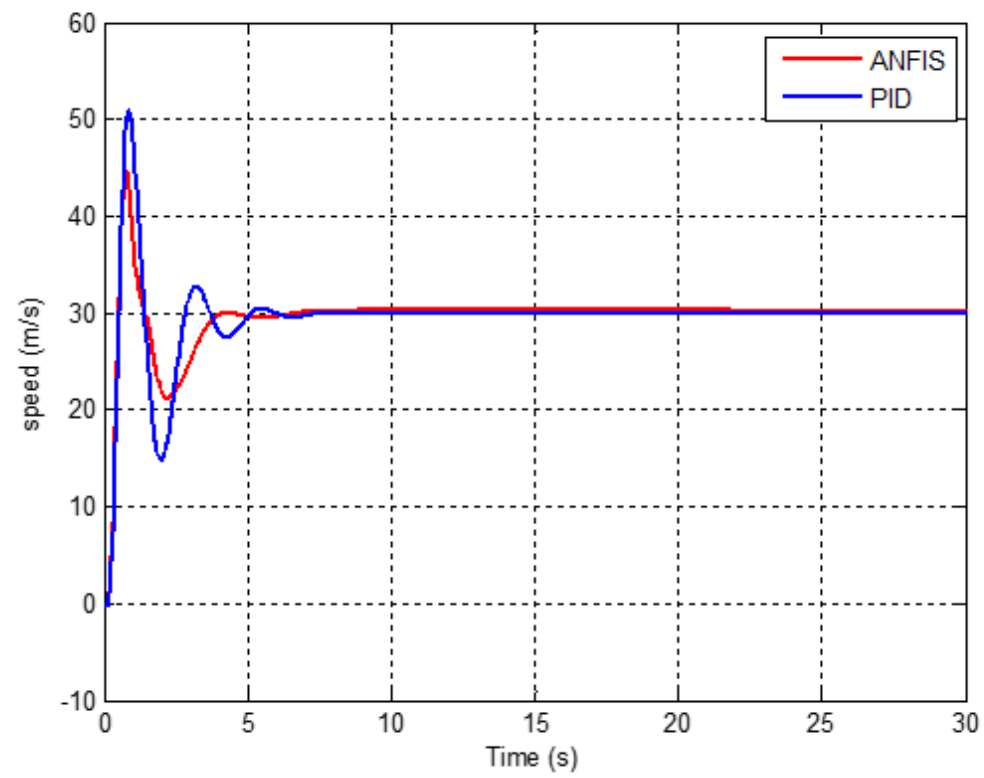

Figure 7.58: Speed-hold longitudinal autopilot response 


\subsubsection{Tracking Different Signal Input}

The ANFIS controller is tested to track different values of the pitch angle, and the response of pitch tracking with \pm 2 values are shown in Fig. 7.59. When the reference altitude is doubled, the proposed ANFIS controller is tested with different altitude values. The response of the altitude control for tracking different altitudes are shown in Fig 7.60, and the speed tracking response at different speed values based on ANFIS are shown in Fig. 7.61.

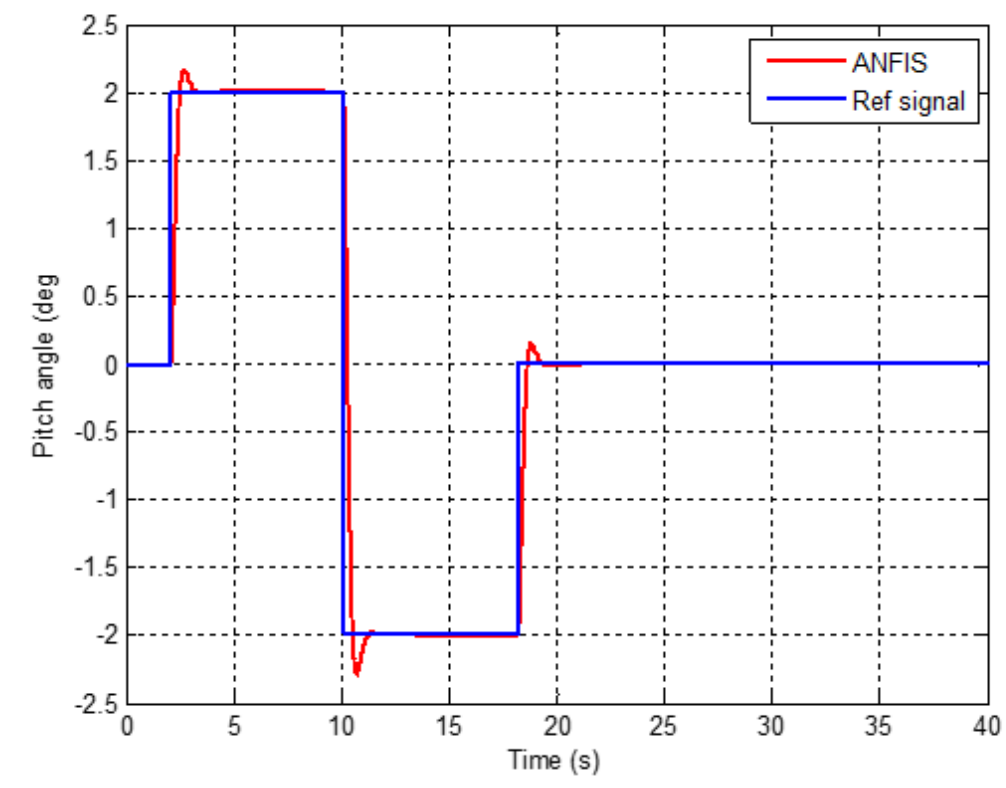

Figure 7.59: ANFIS pitch controller for tracking different signal input 


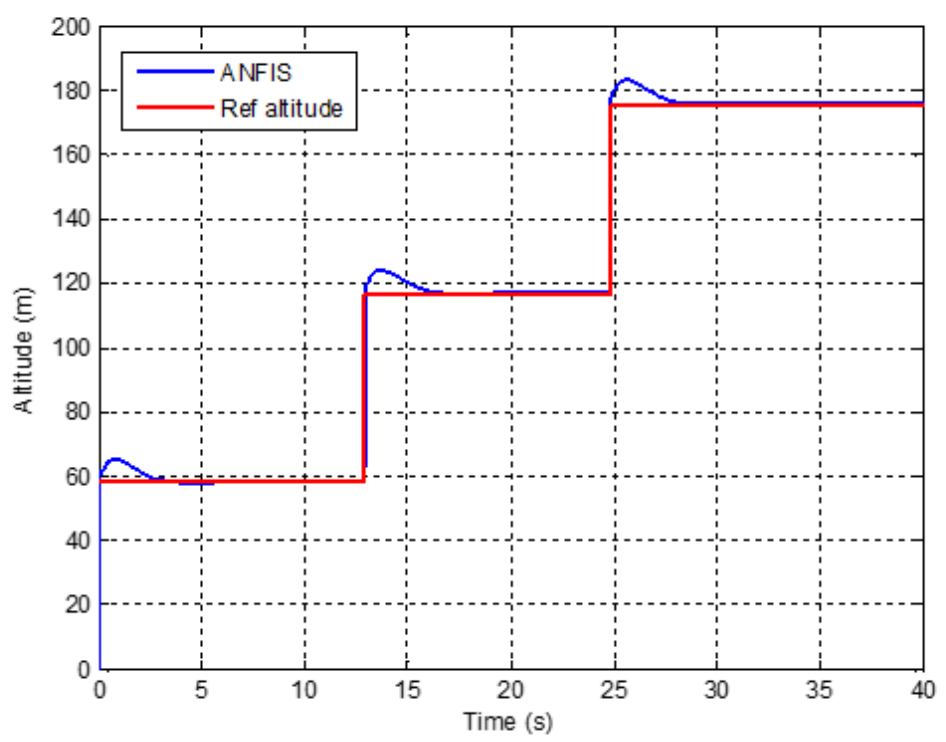

Figure 7.60: ANFIS altitude controller for tracking different altitudese

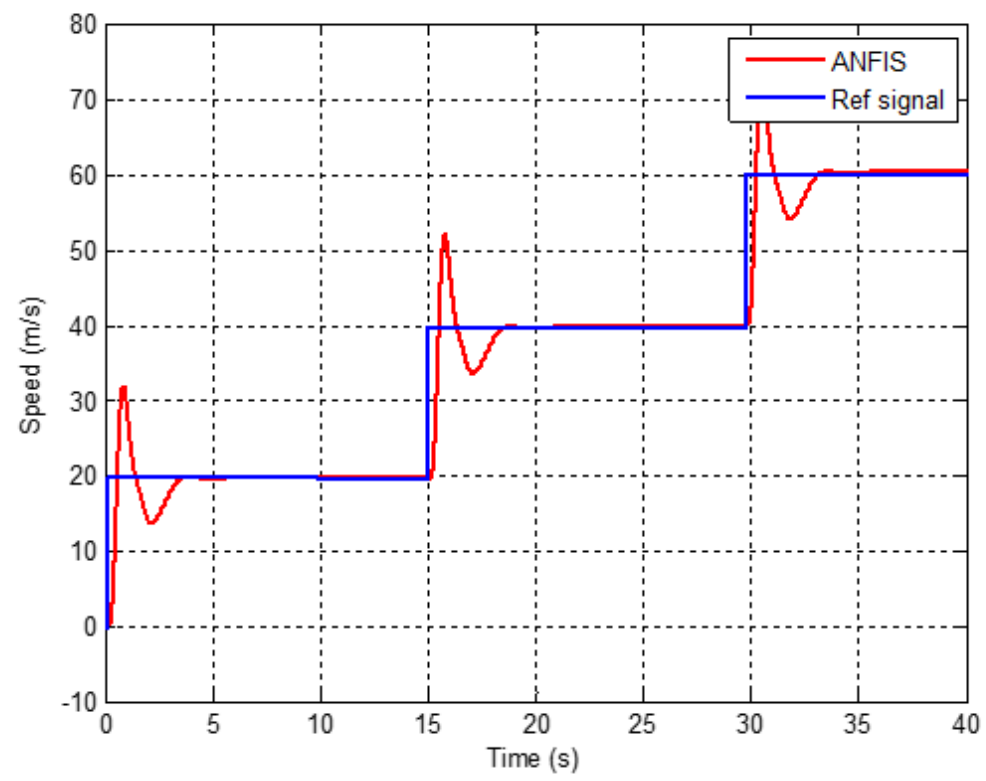

Figure 7.61: ANFIS speed controller for tracking different speeds 


\subsubsection{Reject the Disturbance}

The proposed controller can reject any temporary disturbances. Fig. 7.62 shows the controller response when a disturbance is applied to the system at \pm 2 degrees for three and six seconds for the pitch angle. The ANFIS controller rejects -15 m disturbances at 15 , and 22.5 seconds, as shown in Fig. 7.63 . Fig. 7.64 shows that rejects $+13 \mathrm{~m} / \mathrm{s}$ disturbances at 10 and 20 seconds.

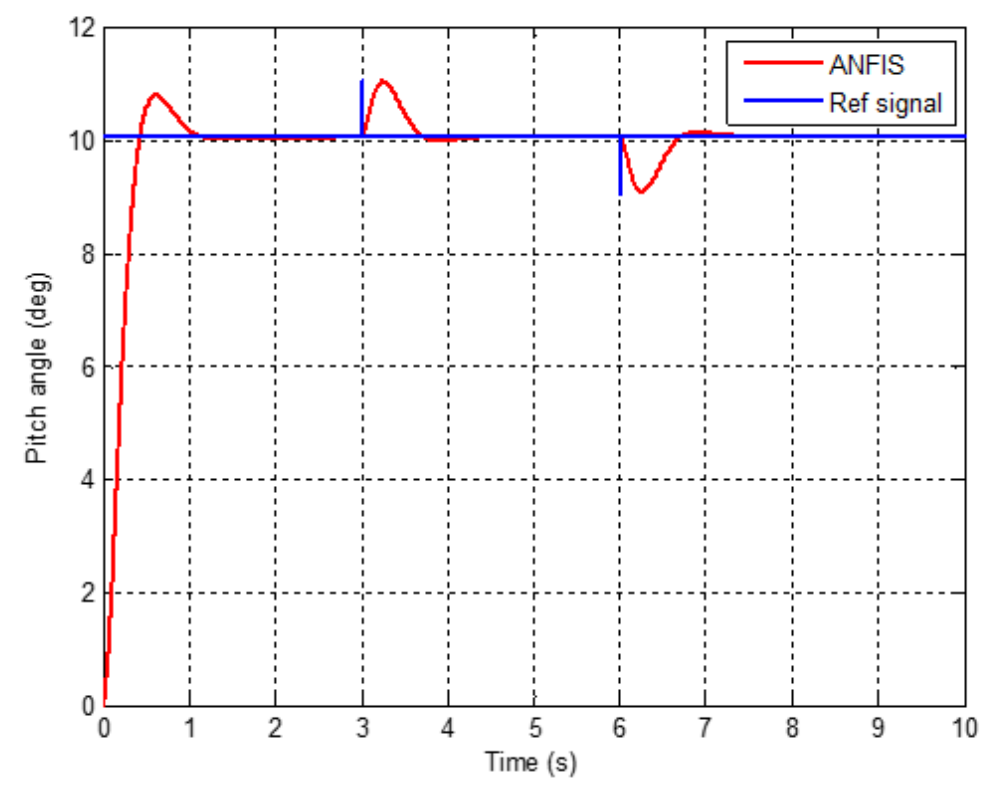

Figure 7.62: The effect of disturbance on pitch tracker 


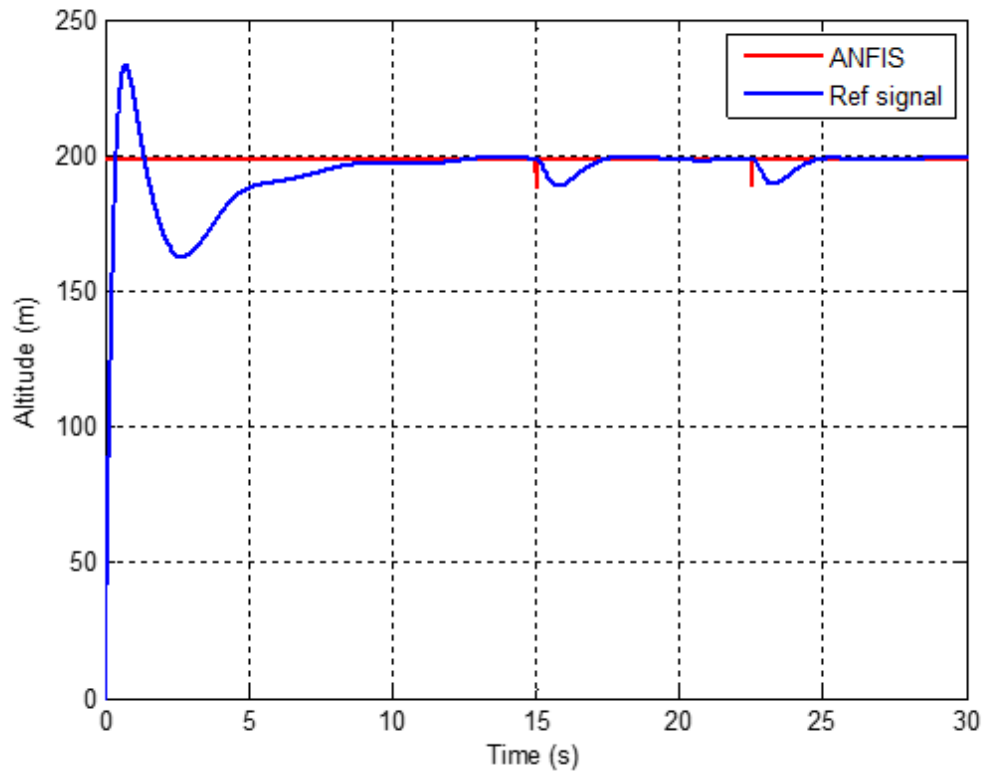

Figure 7.63: The effect of disturbance on altitude

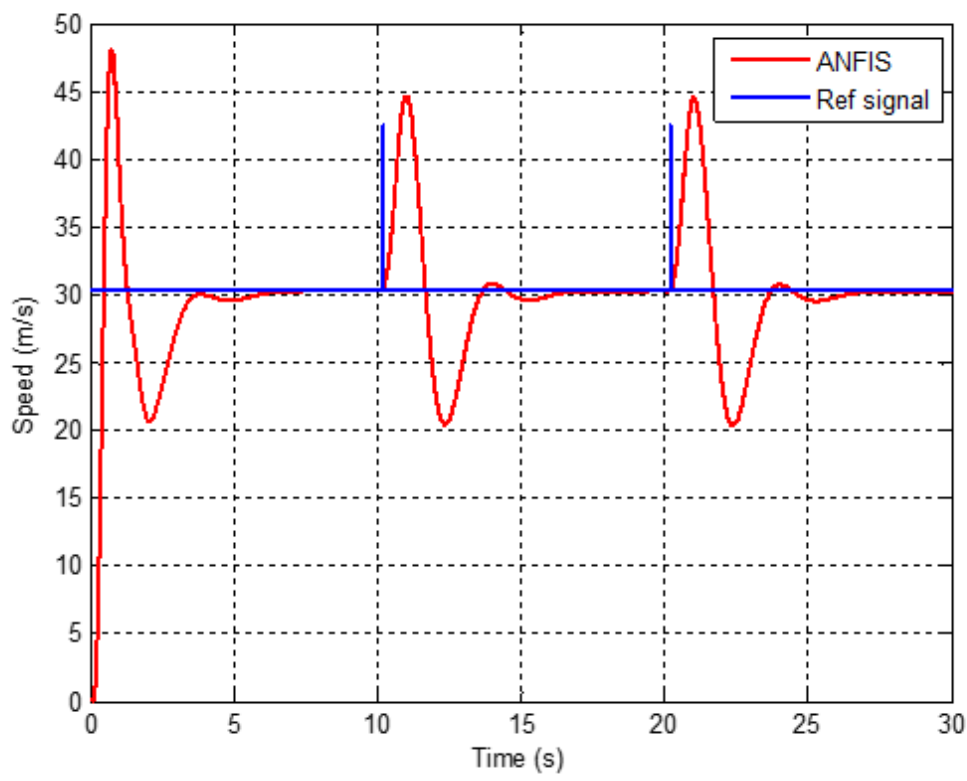

Figure 7.64: The effect of disturbance on speed 


\subsubsection{Lateral Autopilot Simulation}

The lateral autopilot design and testing is discussed in this section. ANFIS and PID controllers are applied to check system performance, and the state-space model is used to design the controllers. The state-space model is given as [4].

$$
\left[\begin{array}{c}
\dot{v} \\
\dot{p} \\
\dot{r} \\
\dot{\phi} \\
\dot{\psi}
\end{array}\right]=\left[\begin{array}{ccccc}
-0.226 & -0.0164 & -9.98 & 0.35 & 0.821 \\
-0.322 & -0.46918 & 0.1632 & 0 & 0 \\
0.0275 & 0.0284 & -0.0289 & 0 & 0 \\
0 & 1 & 0 & 0 & 0 \\
0 & 0 & 1 & 0 & 0
\end{array}\right]\left[\begin{array}{c}
v \\
p \\
\phi \\
\psi
\end{array}\right]+\left[\begin{array}{c}
0 \\
1.263 \\
-0.263 \\
0 \\
0
\end{array}\right] \delta_{\text {lat }}
$$

The state-space matrices are determined using the aerodynamic stability derivatives calculated in Chapter 4, the explanation are provided in Appendix A.The controllability matrix is

$$
C_{n t r}=\left[\begin{array}{lllll}
B & A B & A^{2} B & \ldots & A^{n-1} B
\end{array}\right]
$$

The matrix $C_{n t r}$ has full rank so, the system is controllable.

The inference system for the roll and heading angles are designed as shown in Section 5.3. The roll controller based on the ANFIS and PID controllers is tested to track roll angles from [3 to -3] degrees for 40 seconds, as shown in Fig. 7.65. Their performance is shown in Table7.14. The ANFIS controller has a faster response than the PID with respect to the rising and settling times, and it also give a noticeably better overshoot than the PID controller. The heading control to track different reference angles for 200 seconds is shown in Fig. 7.66. Although, both controllers 
follow the reference angle, the ANFIS has a faster response than the PID, as shown in Table 7.15 .

Table 7.14: Time domain analysis of roll angle

\begin{tabular}{|l|l|l|}
\hline & Classic controller & Designed controller \\
\hline Rise Time(s) & 0.66 & 0.15 \\
\hline Settling Time (s) & 7.96 & 0.46 \\
\hline Overshoot & 5.46 & 5.22 \\
\hline Peak & 1.05 & 1.05 \\
\hline
\end{tabular}

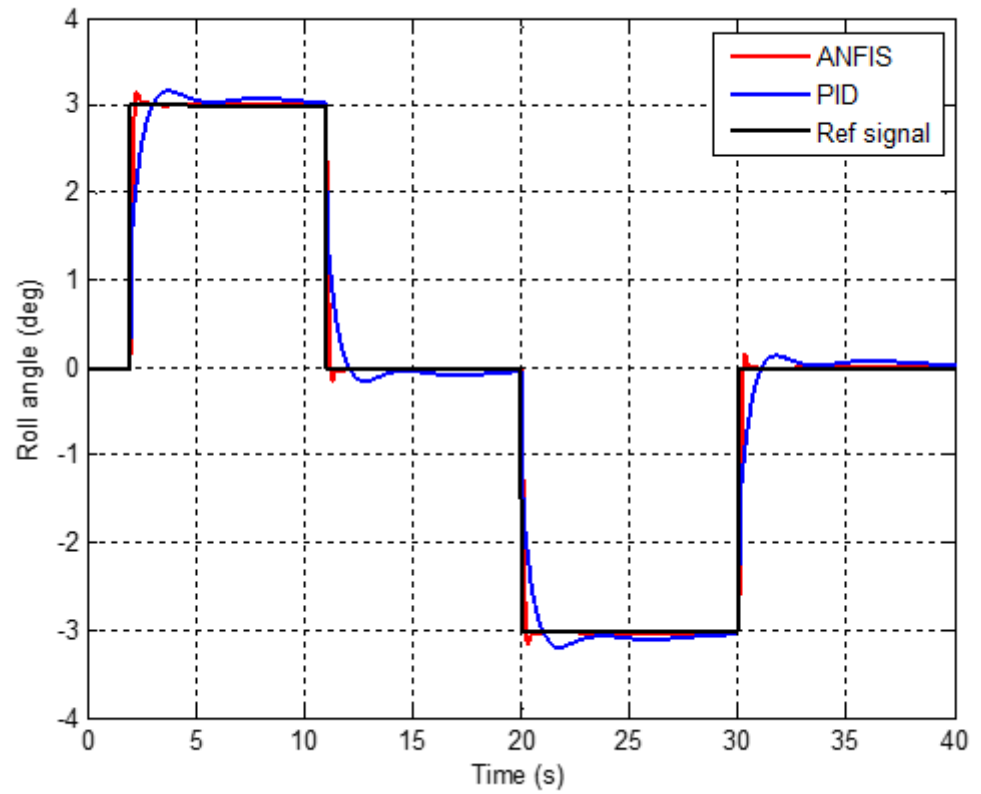

Figure 7.65: Roll controller for tracking different angles 
Table 7.15: Time domain analysis of heading angle

\begin{tabular}{|l|l|l|}
\hline & Classic controller & Designed controller \\
\hline Rise Time(s) & 4.85 & 4.20 \\
\hline Settling Time $(\mathrm{s})$ & 27.2 & 17.6 \\
\hline Overshoot & 14.9 & 12.1 \\
\hline Peak & 1.15 & 1.12 \\
\hline
\end{tabular}

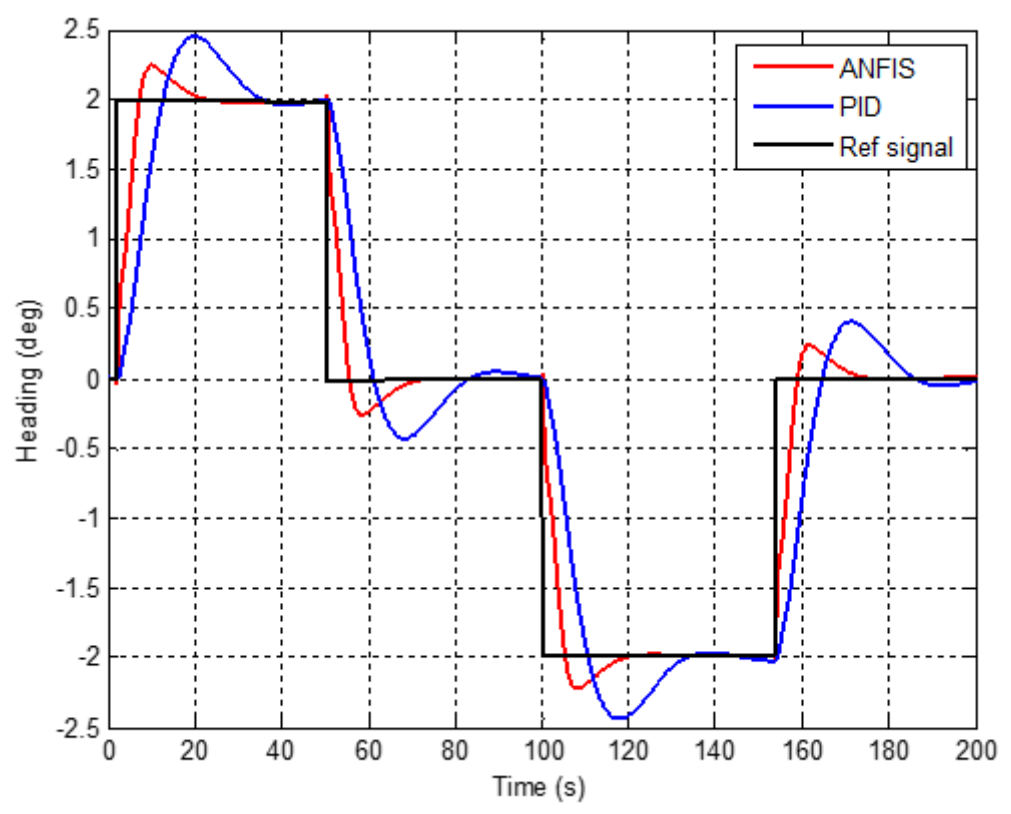

Figure 7.66: Heading controller for tracking different angles

The ANFIS controller can also reject any temporary disturbances, as shown in Figs. 7.67, and 7.68. It rejected disturbance when it was applied at 2 degrees for 10 and 20 seconds in roll angle, and for 40, and 60 seconds in heading. 


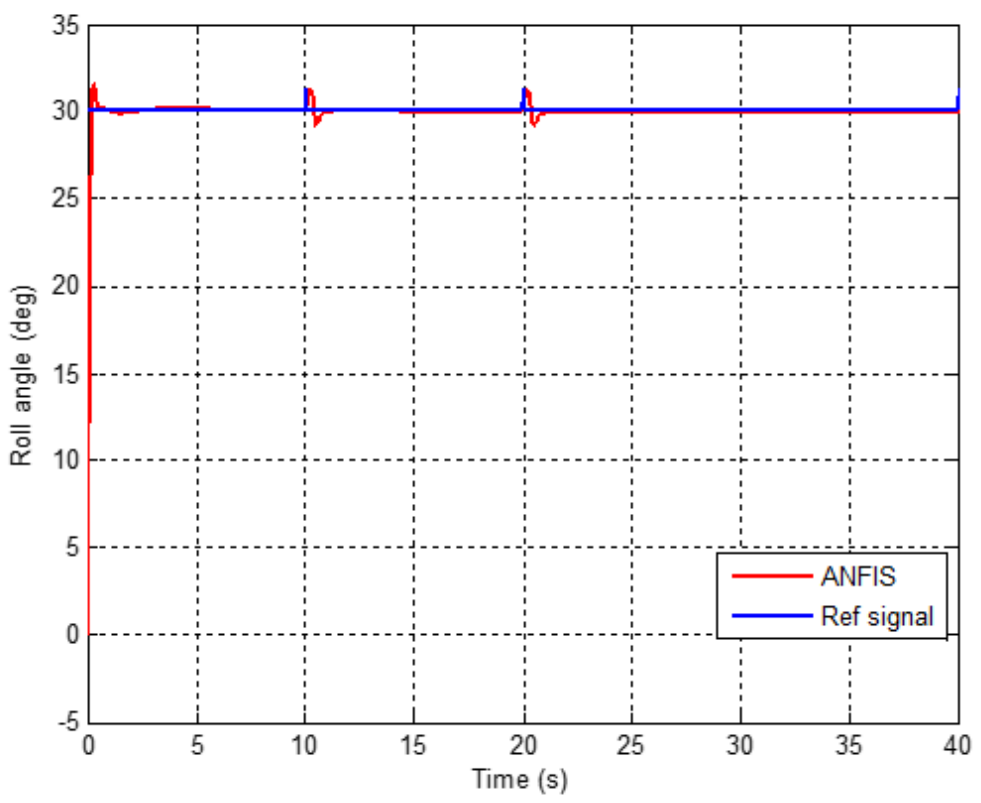

Figure 7.67: The effect of disturbance on roll angle

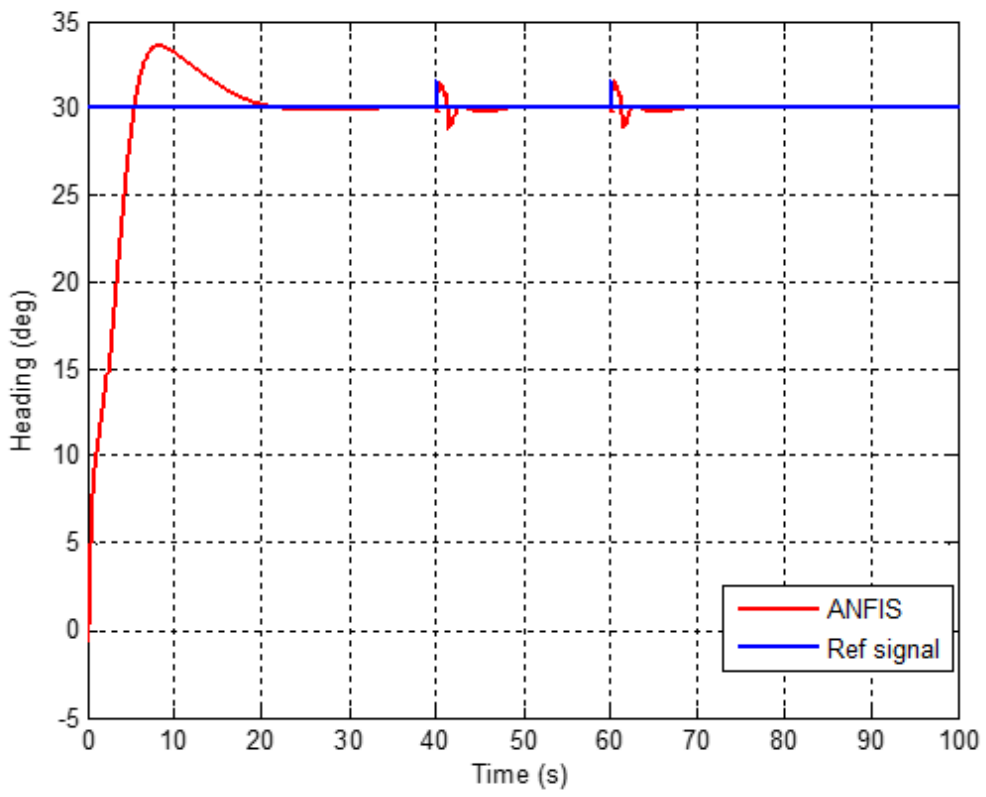

Figure 7.68: The effect of disturbance on heading 


\subsection{Future Works}

There are many potential opportunities to improve on this work, such as:

1. Investigation of different combinations of the wing skin and tracking point colors would enable their simultaneous use as a robust and a fast-visual algorithm for tracking shapes of planar structures.

2. Using another UAV model to validate the performance of the AUKF filter.

3. Use the DDVS system in actual flight tests, and understand that this is the only way to truly validate the control system developed in this thesis.

4. Used DDVS to calculate aerodynamic coefficient parameters and compare them with the experimental test results.

5. Verify the simulation results using flight tests.

6. Use multi-sensors to validate the results, and to estimate all states of a UAV using sensor fusion and AUKF.

7. Increasing autonomous capabilities by adding trajectory planning. 


\section{Chapter 8}

\section{Conclusion}

A new Deflection-Detection Vision System (DDVS) was developed to control a UAV flexible wing, and it is a useful improvement for the UAV control the capabilities. The proposed DDVS includes identifying the flexible wing deflection and classifying its shape to determine the dynamics characteristics for the UAV. The system is comprised of three main elements: a vision system, the fuzzy logic algorithm and an Adaptive Neuro-Fuzzy Inference System ANFIS controller. A stereo camera is employed to improve state estimate and is a key sensor for tasks such as tracking, navigation and positioning. Three dimensional (3D) pose estimation method using planar homography between two images was developed and validated. The literature on homography estimation is quite extensive, and many advanced techniques are available. The Random Sample Consensus (RANSAC) stabilizes noise level results, while the other methods are strongly influenced by them. Furthermore, the smallest and relatively translation error occurred when the noise level is less than 0.5. Three algorithms (Decompose homography, Iterative Closest Point (ICP) and Horns Absolute Orientation method) were applied to determine the accuracy and robustness of each algorithm, and to find the optimal baseline distance between lenses. The dis-

tortion increases according to change in the z-distance or baseline, and are the result 
of a distortion in images or inaccurate camera calibration. As seen in Table 7.2, a baseline of $10 \mathrm{~cm}$ when is more accurate when measuring an object distance equal to or less than $80 \mathrm{~cm}$, while the most accurate baseline is $20 \mathrm{~cm}$ when measuring the distance of more than $80 \mathrm{~cm}$. The vision system uses a ZED stereo camera to find the shape of a flexible wing, and fuzzy logic system was designed to classify the shape of the wing and reveal its dynamic behavior. Several experiments were performed to verify the accuracy of this technique over a broad range of conditions, including wind speed, angle of attack (AOA), and roll angle. The wind tunnel results show that the maximum wing deflection occurred at a wind speed is $31 \mathrm{~m} / \mathrm{s}$ and an AOA equal $20^{\circ}$. Those parameters are used to achieve good system performance when following a desired reference, and the results demonstrate that the vision system is an integral part of an overall control system. The fuzzy logic system used using 18 rules to recognize a suitable wing shape and evaluate the system performance rapidly, and this resulted in a longitudinal and lateral autopilot design for the UAV. Two different techniques were developed for the autopilot design, Adaptive Neuro-Fuzzy Inference System (ANFIS) and a Proportional- Integral- Derivative (PID) controllers. Simulations compared the performance of both controllers with respect to the pitch, speed, roll and heading. The proposed methodology is based on a sensor fusion design to optimize the attitude accuracy and improve processing efficiency. The sensor fusion algorithm was tested and implemented to estimate the attitude of a flexible wing UAV. This method uses information from the Deflection-Detection Vision System (DDVS) and then integrated with data from the accelerometer sensor using a new filter called Adaptive Unscented Kalman Filter (AUKF). This filter employs fuzzy logic to improve the performance by tuning a suitable weighting factor to monitor the AUKF performance quickly. The experimental work was tested and the attitude estimated by the Unscented Kalman Filters (UKF) and AUKF then compared to 
the real value. Experimental results show that the attitude errors are significantly reduced when using the proposed method rather than the UKF estimation method. This techniques demonstrates a real-time solution to attitude estimation for a flexible wing UAV. Furthermore, a data fusion between DDVS and an accelerometer sensor that will improve the state of an UAV was successfully implemented. Environmental disturbances were also considered in the design architecture of the platform. 


\section{List of References}

[1] Rogallo et al. U.S. patent 2546078, [Online]. Available: "https : //en.wikipedia.org/wiki/Francis ${ }_{R}$ ogallo", 2018.

[2] Aero Profi Tl,[Online]. Available: "www.aeros.com.ua/structure/trike/prfT $L_{e}$ n.php", 2008.

[3] U.S. Department of Transportation, FAA-H-8083-38, Airplane Flying handbook, 2016.

[4] M. Spottiswoode, and M. V. Cook, "Modeling the flight dynamics of the hang glider," The Aeronautical Journal, pp. 1-20, 2006.

[5] B. Etkin and D. Lloyd, Dynamics of flight: stability and control, 3rd edition, Wiley, 1996.

[6] P. Dees, "Hang glider design and performance," 10th AIAA Aviation Technology, Integration, and Operations (ATIO) Conference, 2010.

[7] D. F. Dementhon and L. S. Davis, "Model-based object pose in 25 lines of code," International Journal of Computer Vision,, vol. 15, pp. 123-141, 1995.

[8] L. Quan and Z. Lan, "Linear N-point camera pose determination," IEEE Transactions on Pattern Analysis and Machine Intelligence, vol. 21,no. 8, pp. 774-780, 1999.

[9] E. Michaelsen, M. Kirchhof and U. Stilla, "Sensor pose inference from airborne videos by decomposing homography estimates," International Archives of Photogrammetry and Remote Sensing, vol. 35, no. 3, p. 6, 2004.

[10] Y. Ma, S. Soatto, J. Kosecka and S. Sastry, "An invitation to 3-d vision: from images to geometric models," Springer Science and Business Media, vol. 26, 2003. 
[11] D. Faugeras and F. Lustman, "Motion and structure from motion in a piecewise planar environment," International Journal of Pattern Recognition and Artificial Intelligence, vol. 2, pp. 485-508, 1988.

[12] E. Montijano and C. Sagues, "Fast pose estimation for visual navigation using homographies," IEEE/RSJ International Conference in ntelligent Robots and Systems, 2009.

[13] P. J. Besl and N. D. McKay, "A method for registration of 3-D shapes," IEEE Transactions on Pattern Analysis and Machine Intelligence,, vol. 14, no.2, pp. 239-256, 1992.

[14] M. J. Walker and J. Z. Sasiadek, "Accurate pose determination for autonomous vehicle navigation,"18th International Conference in Methods and Models in Automation and Robotics MMAR, 2013.

[15] B. K. Horn, "Closed-form solution of absolute orientation using unit quaternions," Journal of the Optical Society of America, vol. 4, no. 4, pp. 629-642, 1987.

[16] J. Z. Sasiadek and M. J. Walker, "Accurate image depth determination for autonomous vehicle navigation," in CARO3 Conference on Aerospace Robotics, 2015 .

[17] Y. Xu, D. Luo, N. Xian and H. Duan, "Pose estimation for UAV aerial refueling with serious turbulence based on extended kalman filter," Optik-International Journal for Light and Electron Optics, vol. 125, no. 13, pp. 3102-3106, 2014.

[18] V. Kniaz, "Robust vision based pose estimation algorithm for an UAV with known gravity vector," The International Archives of the Photogrammetry, Remote Sensing and Spatial Information Sciences, pp. 63-68, 2016.

[19] T. Weosshaar and H. Ashley, "Static aeroelasticity and the flying wing," Journal of Aircraft, vol. 10, no. 10, pp. 586-594, 1973.

[20] M. J. Patil and D. H. Hodges, "Flight dynamics of highly flexible flying wings," Journal of Aircraft, vol. 43,no. 6, pp. 1790-1798, 2006.

[21] R. Albertani, B. Stanford, J. P. Hubner, R. Lind, and P. Ifju, "Experimental analysis of deformation for flexible-wing micro air vehicles," 46th $A I A A / A S M E / A S C E / A H S / A S C$ Structures, Structural Dynamics and Materials Conference, Austin, 2005. 
[22] A. Burner and T. Liu, "Videogrammetric model deformation measurement technique," Journal of Aircraft,, vol. 38,no. 4, pp. 745-754, 2001.

[23] N. A. Pitcher, J. T. Black, M. F. Reeder, and R. C. Maple, "Videogrammetry dynamics measurements of a lightweight flexible wing in a wind tunnel," Journal of Aircraft, vol. 47, no. 1, pp. 172-180, 2010.

[24] A. W. Burner, R. A. Wahls, and W. K. Goad, "Wing twist measurements at the national transonic facility," NASA Technical Memorandum 110229, 1996.

[25] J. Bakunowicz and R. Meyer, "In-flight wing deformation measurements on a glider," The Aeronautical Journal, vol. 120, no. 1234, pp. 1917-1931, 2016.

[26] A. Burner, T. Liu and R. DeLoach, "Uncertainty of videogrammetric techniques used for aerodynamic testing," 22nd AIAA Aerodynamic Measurement Technology and Ground Testing Conference, 2002.

[27] S. Chiu, S. Chand, D. Moore and A. Chaudhary, "Fuzzy logic for control of roll and moment for a flexible wing aircraft," IEEE Control Systems, vol. 11, no. 4, pp. 42-48, 1991.

[28] M. Keshmiri and W. F. Xie, "Catching moving objects using a navigation guidance technique in a robotic visual servoing system," American Control Conference ACC, pp. 6302-6307, 2013.

[29] G. Rouleau, S. Verma, I. Sharf and E. Martin, "Vision-based tracking and trajectory generation for robotic capture of objects in space," AIAA Guidance, Navigation, and Control Conference and Exhibit, pp. 5937-5950, 2005.

[30] Y. Shi, B. Liang, X. Wang, W. Xu and H. Liu, "Study on intelligent visual servoing of space robot for cooperative target capturing," International Conference Information and Automation (ICIA), pp. 733-738, 2012.

[31] W. Xu, B. Liang, C. Li, Y. Liu and Y. Xu, "Autonomous target capturing of freefloating space robot: Theory and experiments," Robotica, vol. 27, pp. 425-445, 2009.

[32] X. Du, B. Liang, W. Xu and Y. Qiu, "Pose measurement of large non-cooperative satellite based on collaborative cameras," Acta Astronautica, vol. 68, pp. 20472065, 2011. 
[33] R. Zhan and J. Wan, "Neural network-aided adaptive unscented kalman filter for nonlinear state estimation," IEEE Signal Processing Letters, vol. 13, pp. 445-448, 2006.

[34] Z. Jiang, Q. Song, Y. He and J. Han, "A novel adaptive unscented kalman filter for nonlinear estimation," 46th IEEE Conference Decision and Control, pp. 4293-4298, 2007.

[35] C. Hajiyev and H. E. Soken, "Robust adaptive unscented kalman filter for attitude estimation of pico satellites," International Journal of Adaptive Control and Signal Processing, vol. 28, pp. 107-120, 2014.

[36] S. Pourtakdoust and H. Ghanbarpour , "An adaptive unscented Kalman filter for quaternion-based orientation estimation in low-cost AHRS," Aircraft Engineering and Aerospace Technology, vol. 79, no. 5, pp.485-493, 2007.

[37] P. Wahi, R. Raina and F. N. Chowdhury, "A survey of recent work in adaptive flight control," Proceedings of the 33rd Southeastern Symposium on System Theory, 2001.

[38] P. Sujit, S. Saripalli and J. B. Sousa, "Unmanned aerial vehicle path following: A survey and analysis of algorithms for fixed-wing unmanned aerial vehicles," IEEE Control Systems, vol. 34, pp. 42-59, 2014.

[39] H. Bolandi, M. Rezaei, R. Mohsenipour, H. Nemati and S. M. Smailzadeh, " Attitude control of a quadrotor with optimized PID controller," Intelligent Control and Automation, vol. 4, 2013.

[40] A. Sarhan and M. Ashry, "Self-tuned PID controller for the aerosonde UAV autopilot," International Journal of Engineering Research and Technology, vol. 2, pp. 3329-3340, 2013.

[41] P. Shahmaleki and M. Mahzoon, "GA modeling and ANFIS control design for a solar power plant," American Control Conference (ACC), pp. 3530-3535, 2010.

[42] L. S. Brian and L. L. Frank, Aircraft control and simulation, New Jersey: John Wiley and Sons, Inc., 2003.

[43] R. Teo, J. S. Jang and C. J. Tomlin, "Automated multiple UAV flight-the stanford dragonfly UAV program," IEEE Conference on Decision and Control, CDC, 43rd, 2004. 
[44] T. Ng, F. Leung and P. Tam, "A simple gain scheduled PID controller with stability consideration based on a grid-point concept,"Proceedings of the IEEE International Symposium on Industrial Electronics, 1997. ISIE, pp. 1090-1094, 1997.

[45] A. Brezoescu, T. Espinoza, P. Castillo and R. Lozano, "Adaptive trajectory following for a fixed-wing UAV in presence of crosswind," Journal of Intelligent and Robotic Systems, pp. 1-15, 2013.

[46] N. Wahid and N. Hassan, "Self-tuning fuzzy PID controller design for aircraft pitch control," Third International Conference on Intelligent Systems, Modeling and Simulation ISMS, pp. 19-24, 2012.

[47] H. B. Kazemian, "Developments of fuzzy PID controllers," Expert Systems, vol. 22, no. 5, pp. 254-264, 2005.

[48] D. Zhou and Q. Geng, "Multi-model And fuzzy PID Control for fixed-wing UAV," the 3rd International Conference on Mechatronics, Robotics and Automation ICMRA, 2015.

[49] L. M. Argentim, W. C. Rezende, P. E. Santos and R. A. Aguiar, "PID, LQR and LQR-PID on a quadcopter platform," International Conference on Informatics, Electronics and Vision (ICIEV), 2013.

[50] X. J. Xing, J.G. Yan and D.L. Yuan, "Augmented-stability controller design and its simulation for a UAV based on LQR theory," Flight Dynamics, vol. 5, pp. 54-56, 2011.

[51] L. Yibo, C. Chen and W. Chen, "Research on longitudinal control algorithm for flying wing UAV based on LQR technology," International Journal on Smart Sensing and Intelligent Systems, vol. 6, 2013.

[52] S. Kurnaz, O. Cetin and O. Kaynak, "Adaptive neuro-fuzzy inference system based autonomous flight control of unmanned air vehicles," Expert Systems with Applications, vol. 37, pp. 1229-1234, 2010.

[53] A. M. Farid, "UAV cntroller based on adaptive neuro-fuzzy inference system and PID," AES International Journal of Robotics and Automation, vol. 2, 2013.

[54] A. Sarhan and S. Qin, "Autonomous intelligent flight control of fixed-wing UAV based on adaptive neuro-fuzzy inference system," JRET: International Journal of Research in Engineering and Technology, vol. 5, 2016. 
[55] X. Luo, Y. Chen, J. Si and F. Liu, "Longitudinal control of hypersonic vehicles based on direct heuristic dynamic programming using ANFIS," International Joint Conference in Neural Networks (IJCNN), 2014.

[56] G. N. Marichal, A. Hernandez, M. Olivares-Mendez, L. Acosta and P. Campoy, "An intelligent control strategy based on ANFIS techniques in order to improve the performance of a low-cost unmanned aerial vehicle vision system," IEEE/ASME International Conference on Mechatronic and Embedded Systems and Applications, 2010.

[57] C. Loop and Z. Zhang, "Computing rectifying homographies for stereo vision," IEEE Computer Society Conference in Computer Vision and Pattern Recognition, 1999 .

[58] Z. Xuebo, F. Yongchun, M. Bojun, L. Xi and Z. Ming, ”A fast homography decomposition technique for visual servo of mobile robots," 27th Chinese Control Conference CCC, 2008.

[59] M.M. Al-Isawi and J.Z. Sasiadek, "Pose estimation for mobile and flying robots via vision system," Proceedings of the CARO'3 Conference on Aerospace Robotics, 2015.

[60] J. Shi, "Good features to track," Proceedings IEEE Computer Society Conference in Computer Vision and Pattern Recognition, CVPR, 1994.

[61] H. Bay, A. Ess, T. Tuytelaars and L. Van Gool, "Speeded-up robust features (SURF)," Computer Vision and Image Understanding, vol. 110, no. 3, pp. 346359, 2008.

[62] R. Lakemond, S. Sridharan and C. Fookes, "Hessian-based affine adaptation of salient local image features," Journal of Mathematical Imaging and Vision, vol. 44, pp. 150-167, 2012.

[63] M. A. Fischler and R. C. Bolles, "Random sample consensus: a paradigm for model fitting with applications to image analysis and automated cartography," Communications of the ACM, vol. 24, pp. 381-395, 1981.

[64] J. Jae Lee and G. Kim, "Robust estimation of camera homography using fuzzy RANSAC," International Conference on Computational Science and Its Applications, 2007. 
[65] O. Chum and J. Matas,, "Optimal randomized RANSAC," IEEE Transactions on Pattern Analysis and Machine Intelligence, vol. 20, pp. 1472-1482, 2008.

[66] G. Hu, W. MacKunis, N. Gans, W. E. Dixon, J. Chen and A. Behal, "Homography-based visual servo control with imperfect camera calibration," IEEE Transactions on Automatic Control, vol. 54, pp. 1318-1324, 2009.

[67] J. Bouguet, "Camera calibration toolbox for Matlab," [Online]. Available: www.vision.caltech.edu/bouguetj/calib - doc, 2015.

[68] S. Seo, S. Jeonz and S. Lee, "Efficient homography estimation method for panorama," Frontiers of Computer Vision,(FCV), 19th Korea-Japan Joint Workshop , pp. 209-212, 2013.

[69] A. Agarwal, C. Jawahar and P. Narayanan, "A survey of planar homography estimation techniques," Center for Visual Information Technology, Tech. Rep. IIIT/TR, 2005.

[70] A. E. Ahmed, A. Hafez, A. Ouda, H. E. H. Ahmed and H. M. Abd-Elkader, "Modeling of a small unmanned aerial vehicle," International Journal of Mechanical, Aerospace, Industrial and Mechatronics Engineering vol. 9, no. 3, 2015.

[71] M. Chiaramonti, F. Giulietti and G. Mengali, "Formation control laws for a formation of autonomous flight vehicles," 14th Mediterranean Conference Control and Automation, 2006.

[72] M. V. Cook, Flight dynamics principles: A linear systems approach to aircraft stability and control, Butterworth-Heinemann, 2012.

[73] R. C. Nelson, Flight stability and automatic control vol. 2, New York: WCB/McGraw Hill, 1998.

[74] K. Ogata, Modern control engineering, 3rd Ed., Prentice Hall, 1999.

[75] H. Ozbay, Introduction to feedback control theory, CRC Press, 2000.

[76] L. K. Agrawal1, B. K. Chauhan and G. K. Benerjee," A Review on Hybrid Controller Using Soft Computing Algorithms," American Journal of Electrical and Electronic Engineering, vol.4, no. 2, pp 49-61 2016.

[77] J. Ziegler and N. Nichols, "Optimum settings for automatic controllers," Transactions of the ASME, vol.64, pp.759-768, 1942. 
[78] K. H. Ang, G. Chong and Y. Li, "PID control system analysis, design and technology", IEEE Transactions on Control Systems Technology , vol. 13, no. 4, pp. $555-576,2005$.

[79] W.F. Liu, Z. Jiang and Z. Gong, "Online fuzzy self-adaptive PID attitude control of a sub mini fixed-wing air vehicle," IEEE, International Conference on Mechatronics and Automation, pp. 153-157, 2007.

[80] I. Aniekan, O. Ikechukwu, and S. Paul," Comparative analysis of a PID controller using Ziegler Nichols and auto turning method," International Academic Journal of Science and Engineering, vol. 3, no. 10, pp. 1-16, 2016.

[81] J. S. Jang, "ANFIS: adaptive-network-based fuzzy inference system," IEEE Transactions on Systems, Man, and Cybernetics, vol. 23, pp. 665-685, 1993.

[82] S. Mitra and Y. Hayashi, "Neuro-fuzzy rule generation: survey in soft computing framework," IEEE Transactions on Neural Networks, pp. 748-768, 2000.

[83] A. M. Farid, "UAV controller based on adaptive neuro-fuzzy inference system and PID," International Journal of Robotics and Automation, vol. 2, no. 2, pp. 73-82, 2013.

[84] M.A. Denai , F. Palis and A. Zeghbib, "ANFIS based modelling and control of non-linearsSystems : A tutorial," IEEE International Conference on Systems, Man and Cybernetics, 2004.

[85] H. G. de Marina, F. J. Pereda, J. M. Giron-Sierra and F. Espinosa," UAV attitude estimation using unscented Kalman filter and TRIAD," IEEE Transactions on Industrial Electronics, vol. 59, no. 11, 2012.

[86] N. Metni, J. M. Pflimlin, T. Hamel and P. Soueres," Attitude and gyro bias estimation for a flying UAV," IEEE/RSJ International Conference on Intelligent Robots and Systems, pp.1114-1120, 2005.

[87] R.G. Valenti, I. Dryanovski and J. Xiao," A linear kalman filter for marg orientation estimation using the algebraic quaternion algorithm," IEEE Transactions on Instrumentation and Measurement, vol. 65, no. 2, pp. 417-481, 2016.

[88] K. Feng, J. Li, X. Zhang, C. Shen, Y. Bi, T. Zheng and J. Liu, "A new quaternion-based kalman filter for real-time attitude estimation using the twostep geometrically-intuitive correction algorithm," Sensors (Basel), vol. 17, no. $11,2017$. 
[89] D. Titterton and J.L. Weston , Strapdown inertial navigation technology, 2nd Edition. volume 17 of Electromagnetics and Radar Series, 2004.

[90] S. J. Simon and J. K. Jeffrey, "Unscented filtering and nonlinear estimation," Proceedings of the IEEE, vol. 92, no. 3, pp. 401-422, 2004.

[91] J. Z. Sasiadek, Q. Wang and M. B. Zaremba, "Fuzzy adaptive Kalman filtering for INS/GPS data fusion," IEEE International Symposium on Intelligent Control Proceedings, pp. 181-186, 2000.

[92] E.A. Wan and R. Van Der Merwe, "The unscented kalman filter for nonlinear estimation," IEEE Adaptive Systems for Signal Processing, Communications, and Control Symposium, pp. 153-158, 2000.

[93] J. Z. Sasiadek and Q. Wang," Low cost automation using INS/GPS data fusion for accurate positioning," Robotica, vol.21, PP. 255-261,2003.

[94] S. Hung, J. Dah-Jing and W., "Adaptive fuzzy strong tracking extended Kalman Filtering for GPS navigation," IEEE Sensors Journal , vol. 7, no. 5, pp. 778-789, 2007. 


\section{Appendix A}

\section{Equivalent Aerodynamic Stability Derivatives}

The equivelent aerodynamic stability derivatives for the wing and the tricycle [4].

$$
\begin{array}{ll}
\dot{X}_{u} & \left(\dot{X}_{u}\right)_{w}+\left(\dot{X}_{u}\right)_{c} \\
\dot{X}_{w} & \left(\dot{X}_{w}\right)_{w}+\left(\dot{X}_{w}\right)_{c} \\
\dot{X}_{q} & z_{w}\left(\dot{X}_{u}\right)_{w}+z_{c}\left(\dot{X}_{u}\right)_{c}-x_{w}\left(\dot{X}_{w}\right)_{w}-x_{c}\left(\dot{X}_{w}\right)_{c} \\
\dot{Z}_{u} & \left(\dot{Z}_{u}\right)_{w}+\left(\dot{Z}_{u}\right)_{c} \\
\dot{Z}_{w} & \left(\dot{Z}_{w}\right)_{w}+\left(\dot{Z}_{w}\right)_{c} \\
\dot{Z}_{q} & \left(\dot{Z}_{q}\right)_{w}+z_{w}\left(\dot{Z}_{u}\right)_{w}+z_{c}\left(\dot{Z}_{u}\right)_{c}-x_{w}\left(\dot{Z}_{w}\right)_{w}-x_{c}\left(\dot{Z}_{w}\right)_{c} \\
\dot{M}_{u} & \left(\dot{M}_{u}\right)_{w}-x_{w}\left(\dot{Z}_{u}\right)_{w}+z_{w}\left(\dot{X}_{u}\right)_{w}-x_{c}\left(\dot{Z}_{u}\right)_{c}+z_{c}\left(\dot{X}_{u}\right)_{c} \\
\dot{M}_{w} & \left(\dot{M}_{w}\right)_{w}-x_{w}\left(\dot{Z}_{w}\right)_{w}+z_{w}\left(\dot{X}_{w}\right)_{w}-x_{c}\left(\dot{Z}_{w}\right)_{c}+z_{c}\left(\dot{X}_{w}\right)_{c} \\
\dot{M}_{q} & \left(\dot{M}_{q}\right)_{w}+\left(\dot{M}_{q}\right)_{c}+z_{w}\left(\dot{M}_{u}\right)_{w}-x_{w}\left(\dot{M}_{w}\right)_{w}-x_{w}\left(\dot{Z}_{q}\right)_{w}-x_{w} z_{w}\left(\dot{Z}_{u}\right)_{w}+x_{w}^{2}\left(\dot{Z}_{w}\right)_{w} \\
& +z_{w}^{2}\left(\dot{X}_{u}\right)_{w}-x_{w} z_{w}\left(\dot{X}_{w}\right)_{w}-x_{c} z_{c}\left(\dot{Z}_{u}\right)_{c}+x_{c}^{2}\left(\dot{Z}_{w}\right)_{c}+z_{c}^{2}\left(\dot{X}_{u}\right)_{C}-x_{c} z_{c}\left(\dot{X}_{w}\right)_{c}
\end{array}
$$




$$
\begin{array}{ll}
\dot{Y}_{v} & \left(\dot{Y}_{v}\right)_{w}+\left(\dot{Y}_{v}\right)_{c} \\
\dot{Y}_{p} & -z_{w}\left(\dot{Y}_{v}\right)_{w}+z_{c}\left(\dot{Y}_{v}\right)_{c} \\
\dot{Y}_{r} & x_{w}\left(\dot{Y}_{v}\right)_{w}+x_{c}\left(\dot{Y}_{v}\right)_{c} \\
\dot{L}_{v} & \left(\dot{L}_{v}\right)_{w}-z_{w}\left(\dot{Y}_{v}\right)_{w}-z_{c}\left(\dot{Y}_{v}\right)_{c} \\
\dot{L}_{p} & \left(\dot{L}_{p}\right)_{w}-z_{w}\left(\left(\dot{L}_{v}\right)_{w}-z_{w}\left(\dot{Y}_{v}\right)_{w}\right)+z_{c}^{2}\left(\dot{Y}_{v}\right)_{c} \\
\dot{L}_{r} & \left(\dot{L}_{r}\right)_{w}+x_{w}\left(\dot{Y}_{v}\right)_{w}+x_{c}\left(\dot{Y}_{v}\right)_{c} \\
\dot{N}_{v} & \left(\dot{N}_{v}\right)_{w}+x_{w}\left(\dot{Y}_{v}\right)_{w}+x_{c}\left(\dot{Y}_{v}\right)_{c} \\
\dot{N}_{p} & \left(\dot{N}_{p}\right)_{w}-z_{w}\left(\left(\dot{N}_{v}\right)_{w}-x_{w}\left(\dot{Y}_{v}\right)_{w}\right)-x_{c} z_{c}\left(\dot{Y}_{v}\right)_{c} \\
\dot{N}_{r} & \left(\dot{N}_{r}\right)_{w}+\left(\dot{N}_{r}\right)_{c}+x_{w}\left(\left(\dot{N}_{v}\right)_{w}-x_{w}\left(\dot{Y}_{v}\right)_{w}\right)+x_{c}^{2}\left(\dot{Y}_{v}\right)_{c}
\end{array}
$$

Table A.1: The longitudenal derivatives for the wing

\begin{tabular}{|l|l|l|l|}
\hline & \multicolumn{4}{|l|}{ Longitudinal Dimensional Derivatives } \\
\hline & $\mathrm{X}$ & $\mathrm{Z}$ & $\mathrm{M}$ \\
\hline $\mathrm{u}$ & $\rho u_{0} S C_{w} \sin \theta+\frac{1}{2} \rho u_{0} S C_{x u}$ & $-\rho u_{0} S C_{w} \cos \theta+\frac{1}{2} \rho u_{0} S C_{z u}$ & $\frac{1}{2} \rho u_{0} \bar{c} S C_{m u}$ \\
\hline $\mathrm{w}$ & $\frac{1}{2} \rho u_{0} S C_{x \alpha}$ & $\frac{1}{2} \rho u_{0} S C_{z \alpha}$ & $\frac{1}{2} \rho u_{0} \bar{c} S C_{m \alpha}$ \\
\hline $\mathrm{q}$ & $\frac{1}{4} \rho u_{0} \bar{c} S C_{x q}$ & $\frac{1}{4} \rho u_{0} \bar{c} S C_{z q}$ & $\frac{1}{4} \rho u_{0} \bar{c}^{2} S C_{m q}$ \\
\hline
\end{tabular}


APPENDIX A. EQUIVALENT AERODYNAMIC STABILITY DERIVATIVES 135

Table A.2: The lateral derivatives for the wing

\begin{tabular}{|l|l|l|l|}
\hline & \multicolumn{3}{|l|}{ Lateral Dimensional Derivatives } \\
\hline & $\mathrm{Y}$ & $\mathrm{L}$ & $\mathrm{N}$ \\
\hline $\mathrm{v}$ & $\frac{1}{2} \rho u_{0} S C_{y \beta}$ & $\frac{1}{2} \rho u_{0} b S C_{l \beta}$ & $\frac{1}{2} \rho u_{0} b S C_{n \beta}$ \\
\hline $\mathrm{p}$ & $\frac{1}{4} \rho u_{0} b S C_{y p}$ & $\frac{1}{4} \rho u_{0} b^{2} S C_{l p}$ & $\frac{1}{4} \rho u_{0} b^{2} S C_{n p}$ \\
\hline $\mathrm{r}$ & $\frac{1}{4} \rho u_{0} b S C_{y r}$ & $\frac{1}{4} \rho u_{0} b^{2} S C_{l r}$ & $\frac{1}{4} \rho u_{0} b^{2} S C_{n r}$ \\
\hline
\end{tabular}

- The Longitudinal Derivatives for tricycle are:

$$
\begin{aligned}
& X_{u c}=\frac{1}{2} \rho S \frac{w_{e q}^{2}}{u_{e q}} C_{T u} \cos \delta_{e q}+\frac{1}{2} \rho S u_{e q}\left[-2\left(C_{D p}\right)_{e q} \cos \alpha_{e q}+\left(2 C_{T}+C_{T u}\right) \cos \delta_{e q}\right] \\
& Z_{u c}=\frac{1}{2} \rho S \frac{w_{e q}^{2}}{u_{e q}} C_{T u} \sin \delta_{e q}+\frac{1}{2} \rho S u_{e q}\left[-2\left(C_{D p}\right)_{e q} \sin \alpha_{e q}+\left(2 C_{T}+C_{T u}\right) \sin \delta_{e q}\right] \\
& M_{u c}=0 \\
& X_{w c}=\frac{1}{2} \rho S w_{e q}\left[-2\left(C_{D p}\right)_{e q} \cos \alpha_{e q}+2 C_{T} \cos \delta_{e q}\right]+\frac{1}{2} \rho S\left(u_{e q}+\frac{w_{e q}^{2}}{u_{e q}}\right) C_{D p} \sin \alpha_{e q} \\
& Z_{u c}=\frac{1}{2} \rho S w_{e q}\left[-2\left(C_{D p}\right)_{e q} \sin \alpha_{e q}+2 C_{T} \sin \delta_{e q}\right]+\frac{1}{2} \rho S\left(u_{e q}+\frac{w_{e q}^{2}}{u_{e q}}\right) C_{D p} \cos \alpha_{e q} \\
& M_{w c}=0 \\
& X_{q c}=0 \\
& Z_{q c}=0 \\
& M_{q c}=0
\end{aligned}
$$

- The Lateral Derivatives for tricycle are: 


$$
\begin{aligned}
& \left.Y_{v c}=\frac{1}{2} \rho S\left(u_{e q}+\frac{w_{e q}^{2}}{u_{e q}}\right)\left[-2\left(C_{D p}\right)_{e q} \cos \alpha_{e q}-C_{T u}\right) \cos \delta_{e q}\right] \\
& L_{v c}=0 \\
& N_{v c}=0 \\
& Y_{p c}=0 \\
& Y_{r c}=0 \\
& L_{p c}=0 \\
& L_{r c}=0 \\
& N_{p c}=0 \\
& N_{r c}=0
\end{aligned}
$$

- The thrust Control Derivatives are:

$$
\begin{aligned}
X_{\delta t h} & =\frac{1}{2} \rho S C_{T \delta t h} \cos \delta_{e q} \\
L_{\delta t h} & =0 \\
Y_{\delta t h} & =0 \\
M_{\delta t h} & =0 \\
Z_{\delta t h} & =\frac{1}{2} \rho S C_{T \delta t h} \sin \delta_{e q} \\
N_{\delta t h} & =0
\end{aligned}
$$

- The equivalent aerodynamic control derivatives are:

$$
\begin{aligned}
& L_{\xi}=\frac{m_{c} l_{2}}{m} L_{w} \cos (\delta) \cos (\alpha) \\
& M_{\delta}=\frac{m_{c} l_{2}}{m} \cos (\delta)\left(L_{w} \cos (\delta-\alpha)+\left(D_{w}-\frac{m_{w}}{m_{c}} D_{c}\right) \sin (\delta-\alpha)\right) \\
& N_{\xi}=0
\end{aligned}
$$

